\title{
Alzheimer's Disease is Driven by Beta-Amyloid Generated in the Amyloid Precursor Protein-Independent Pathway and Retained Intraneuronally: Research and Therapeutic Strategies in a New AD Paradigm
}

\author{
Vladimir Volloch $^{{ }^{*}}$ and Sophia Rits-Volloch ${ }^{2,3}$ \\ 'Department of Developmental Biology, Harvard School of Dental Medicine, Boston, USA \\ ${ }^{2}$ Division of Molecular Medicine, Children's Hospital, Boston, USA \\ ${ }^{3}$ Department of Biological Chemistry and Molecular Pharmacology, Harvard Medical School, Boston, USA
}

\begin{abstract}
The present article describes a New Paradigm of Alzheimer's disease (AD). In the Old Paradigm, formalized in the Amyloid Cascade Hypothesis (ACH) theory of $\mathrm{AD}$, beta amyloid $(\mathrm{A} \beta)$ is produced, both in health and disease, solely in the amyloid precursor protein ( $\beta \mathrm{APP})$ proteolytic/secretory pathway. Two $\beta \mathrm{APP}$ cleavages are involved. First cleavage, by beta-secretase (Beta-site APP Cleaving Enzyme, BACE) between Met671 and Asp672 (numbering according to the $\beta$ APP770 isoform), generates the C-terminal fragment of $\beta A P P$ (C99, reflecting the number of its amino acid residues) and forms the $\mathrm{N}$-terminus of $A \beta$. Subsequent second cleavage of $\mathrm{C} 99$ by gamma-secretase (gamma-site $\beta$ APP cleaving enzyme) forms the $\mathrm{C}$-terminus of $\mathrm{A} \beta$, completes its production, and coincides with its secretion. The overproduction of $A \beta$ results in its extracellular accumulation commencing early in life. This triggers a cascade of molecular and cellular events, including formation of neurofibrillary tangles, which results in neurodegeneration. When the extent of neurodegeneration reaches critical levels, symptoms of the disease are manifested. In this Paradigm, Alzheimer's disorder is a quintessential "slow" disease. The ACH clearly defined therapeutic targets, which included key events of $\beta$ APP proteolysis as well as secreted extracellular A $\beta$. Eventually, a number of candidate AD drugs, highly effective in animal model systems, was developed. Of those, especially successful were inhibitors of beta-sectretase that not only prevented the emergence of AD symptoms, but also reversed them when administered after symptomatic manifestation of the disease in animal models. At this point, there was every reason to hope that a solution to the Alzheimer's problem is at hand; this, however proved not to be the case. Both the Old and the New Paradigms share the common point of departure, namely that the overproduction of beta-amyloid is the causative basis of AD. The rest of the notions of the New Paradigm are distinctly different from those of the Old one. Formulation of the New Paradigm theory of Alzheimer's disease was necessitated by the analysis of results of massive human clinical trials of candidate AD drugs that performed outstandingly in animal studies. They all failed in human trials as spectacularly as they succeeded in animal studies. Or did they? Whereas they indeed showed no efficacy whatsoever, they performed perfectly within confines of their design and purpose. For example, a BACE inhibitor verubecestat penetrated the brain of $\mathrm{AD}$ patients, greatly inhibited $\beta$ APP cleavage, and strongly suppressed extracellular levels of A $\beta$. It did all this with the same efficiency it exhibited in animal studies, where it indisputably succeeded in mitigating symptoms of the disease. Why did it fail to do so in human clinical trials? This failure, apparently inexplicable within the confines of the $\mathrm{ACH}$, seems as good an occasion as any to apply the central dictum of Sherlock Holmes: "... when you eliminated the impossible, whatever remains, however improbable, must be the truth". In the case under discussion, after the elimination of the "impossible", including elements of the ACH, and provided that Alzheimer's disease is indeed caused by the overproduction of $A \beta$, a notion strongly supported by experimental data, the "however improbable", which is consistent with the outcomes of human clinical trials of AD candidate drugs, is the following. 1. In addition to the $\beta$ APP proteolytic/secretory process, in Alzheimer's disease in humans, $A \beta$ is also produced in the $\beta$ APP-independent pathway. This pathway is active only in AD patients. It does not operate in animals and healthy humans. 2 . The output of the $\beta$ APP-independent A $\beta$ generation pathway is retained intraneuronally, and it is this pool of intraneuronal beta-amyloid that causes and sustains Alzheimer's disease. These notions constitute the core of the New Paradigm theory of AD. Several mechanisms are capable of achieving the above. They include: RNA-dependent $\beta A P P$ mRNA amplification, a process implicated in overproduction of specific proteins in mammalian cells; the internal initiation of transcription within the human $\beta$ APP gene; cleavage within $\beta$ APP mRNA; the internal initiation of translation within $\beta$ APP mRNA. Conceptually, in
\end{abstract}

Citation: Volloch V, Rits-Volloch S. Alzheimer's Disease is Driven by Beta-Amyloid Generated in the Amyloid Precursor Protein-Independent Pathway and Retained Intraneuronally: Research and Therapeutic Strategies in a New AD Paradigm. Ann Integr Mol Med. 2021; 2(1): 1010.

Copyright: $\odot 2021$ Vladimir Volloch

Publisher Name: Medtext Publications LLC

Manuscript compiled: Sep $08^{\text {th }}, 2021$

${ }^{*}$ Corresponding author: Vladimir Volloch, Department of Developmental Biology, Harvard School of Dental Medicine, USA, E-mail: vladimir.volloch@gmail.com; vladimir_volloch@hms.harvard.edu

Contact information: Sophia Rits-Volloch, rits@crystal.harvard.edu the context of the present article, the nature of the mechanism generating $A \beta$ independently of $\beta \mathrm{APP}$ is not important; in every case, identical therapeutic strategies would be indicated. All potential mechanisms of $\beta$ APP-independent $\mathrm{A} \beta$ generation share several common features. (a) In each case, the expression of a crucial component(s) required for activation of a specific mechanism is induced by the integrated stress response (ISR) elicited via OMA1-DELE1HRI signaling pathway activated by mitochondrial dysfunction triggered by over-the-threshold levels of $\beta$ APP-derived $A \beta$ accumulated intracellularly through cellular uptake of secreted $A \beta$ as well as by retention of a fraction of beta-amyloid produced in the $\beta$ APP proteolytic pathway. (b) In every potential mechanism of $\beta A P P$-independent $A \beta$ generation, translation initiates at the AUG normally encoding Met 671 of $\beta$ APP and results in C100, i.e. N-terminal Met-containing $\mathrm{C} 99$, which is subsequently cleaved by gamma-secretase to produce $A \beta$ (or Met-A $\beta$ ). (c) N-terminal Met of C100 is removed postrather than co-translationally. Therefore a steady-state population of C100 and, possibly, of $\mathrm{N}$-terminal Met-containing $\mathrm{A} \beta$ should occur within human 
neuronal cells with the activated $\beta$ APP-independent $\mathrm{A} \beta$ production pathway; their detection would provide irrefutable proof of operation of the pathway. (d) The $\mathrm{A} \beta$ (or Met- $\mathrm{A} \beta$ ) output of every potential mechanism is retained intraneuronally. (e) Once activated, every potential $\beta$ APP-independent mechanism would, through generation of intraneuronally retained $\mathrm{A} \beta$, sustain mitochondrial dysfunction and support the activity of the OMA1-DELE1-HRI signaling pathway, which, in turn, will promote, via elicitation of the ISR, the operation of the $\beta$ APP-independent $A \beta$ production pathway. These self-perpetuating $\{\beta A P P-i n d e p e n d e n t$ generation of intracellularly retained $A \beta\} /\{$ mitochondrial dysfunction\} mutual feedback cycles constitute the "Engine" that drives Alzheimer's disease. The lifelong accumulation of intraneuronal $\beta$ APP-derived A $\beta$ to critical levels, sufficient to trigger mitochondrial dysfunction, plays the role of a starter motor in getting car engine moving in a self-sustainable manner. Only when the AD "Engine" is activated does the disease commence. In the New Paradigm, therefore, Alzheimer's disorder is a "fast" disease that can be treated and cured at the symptomatic stages. The present article proposes conceptually novel research and therapeutic strategies and suggests that BACE activation (yes, activation!) could be a valid approach in AD therapy.

Keywords: $\beta$ APP-independent generation of intraneuronally retained A $\beta$; Mitochondrial distress; OMA1-DELE1-HRI-ISR signaling pathway; Selfperpetuating AD "Engine"; N-terminal Met-C99 (C100); N-terminal Met-A $\beta$; Research and therapeutic strategies in a New Alzheimer's Paradigm

\section{Introduction}

The prevailing cause of dementia, Alzheimer's disease, AD, was, until recently, believed to be initiated and driven by the extracellular accumulation of beta-amyloid, $A \beta$, shown to be overproduced in the disease. This peptide, it was assumed, is derived, both in health and disease, solely by two proteolytic cleavages of a large beta-amyloid precursor protein, $\beta$ APP, which occur in the secretory pathway. First cleavage, by beta-secretase (Beta-site APP Cleaving Enzyme, BACE) between residues 671 and 672 (numbering according to the $\beta A P P 770$ isoform), generates the C-terminal fragment of $\beta$ APP (C99, reflecting the number of its amino acid residues) and forms the $\mathrm{N}$-terminus of $\mathrm{A} \beta$. Subsequent second cleavage of C99 by gamma-secretase (gamma-site $\beta$ APP cleaving enzyme) forms the C-terminus of $A \beta$ and completes its production; the resulting beta-amyloid peptide is then secreted into the extracellular space. In $\mathrm{AD}$, according to this view, $\mathrm{A} \beta$ overproduction and associated damages begin early, accumulate slowly throughout the lifespan, and manifest symptomatically late in life in sporadic cases, SAD, or around the fifties in familial cases, FAD; in this, the "Old", Paradigm, Alzheimer's disorder is a quintessential "slow" disease. These notions were formalized in the Amyloid Cascade Hypothesis, $\mathrm{ACH}$, which became a prevailing evidence-based theory of $\mathrm{AD}$, and $\mathrm{A} \beta$ emerged as the most compelling therapeutic target of the disease. In recent years, a large number of potential AD drugs, targeting either the generation of beta-amyloid via the $\beta$ APP proteolytic pathway or the extracellular $A \beta$ directly, has been developed. They include inhibitors of beta-secretase such as atabecestat, lanabecestat, AZD3839, LY2811376, LY2886721, verubecestat; inhibitors and modulators of gamma-secretase such as avagacestat, begacestat, semagacestat, tarenflurbil; inhibitors of beta-amyloid aggregation such as scillo-inositol, PBT2, tramiprosate; $\mathrm{A} \beta$-based antigens such as vanutide, $\mathrm{AD} 02, \mathrm{CAD}-106, \mathrm{AN}-1792$; and both polyclonal and monoclonal $\mathrm{A} \beta$-targeting antibodies such as crenezumab, gantenerumab, solanezumab, bapineuzumab and ponezumab. Many of these potential drugs achieved spectacular successes in treatment, even the reversal, of AD symptoms in animal models. They all, however, exhibited equally spectacular failures in human clinical trials. To explain this discordance, it was suggested that the drugs were administered too late in the course of the disease in human trials. Therefore, the trials, initially involving mildto-moderate $\mathrm{AD}$ patients, were repeated with patients suffering from mild cognitive impairment and biomarker evidence of $A \beta$ deposition in brain (prodromal AD). The outcomes, however, did not change; moreover, in a number of cases the administration of drugs actually worsened the cognitive or clinical condition of the subjects. This was explained away by suggesting that even the prodromal cases are way too advanced for treatment because irreversible $A \beta$-triggered damages have been accumulating for decades, presumably starting in the early twenties, and, to be effective, this is when therapeutic intervention should commence and continue the entire life.

The determination that all $\mathrm{AD}$ candidate drugs failed in human clinical trials is, however, conditional and requires some explaining. Indeed, all candidate drugs failed in that they neither relieved the $\mathrm{AD}$ symptoms nor provided any clinical improvement to the patients. On the other hand, they, or at least some of them, succeeded remarkably within confines of their design and purpose and within the framework of the $\mathrm{ACH}$. To appreciate this, it is instructional to dissect the case of the verubecestat, a MERK-developed BACE inhibitor. Its two human clinical stage III trials were concluded prematurely because of the complete lack of efficacy. Yet at the mechanistic level it was, unquestionably, a success. Treatment with verubecestat led to decrease of extracellular deposition of $\mathrm{A} \beta$ in brains of $\mathrm{AD}$ patients and to a drastic reduction, over $80 \%$, of its levels in the CSF [1]. Verubecestat was designed to penetrate the brain, to suppress beta-secretase cleavage of $\beta$ APP and, consequently, generation and secretion of beta-amyloid in $\mathrm{AD}$. And this is precisely what it did, and it did it very effectively, in human patients. Conceptually similar results were obtained with candidate drugs targeting secreted $A \beta$, such as beta-amyloid antibodies, which led to the reduction of extracellular $A \beta$ deposits. Thus, the candidate drugs failed clinically, because of the lack of efficacy, but they succeeded mechanistically and did it with the same efficiency they exhibited in animal studies, where they were successful in mitigating symptoms of the disease. Why did they fail to do so in human clinical trials? Provided that the $\mathrm{ACH}$ is correct in its departure point notion, namely that $A \beta$ initiates a cascade of molecular and cellular events resulting in $\mathrm{AD}$, a concept strongly supported by experimental data, the results of massive human clinical trials suggest two breakthrough insights: 1. In addition to the $\beta$ APP proteolytic/secretory pathway of $A \beta$ production, in Alzheimer's disease beta-amyloid is also generated in the $\beta$ APPindependent manner (by whatever mechanism). 2. The entire output of $A \beta$ produced in the $\beta$ APP-independent pathway, or the bulk, or at least a substantial fraction of it, is not secreted but is retained intraneuronally, and it is this, intraneuronally retained, pool of beta-amyloid that causes and sustains Alzheimer's disease. These notions constitute the core of the New Paradigm of AD. The above reasoning necessitated a comprehensive reassessment, detailed below, of our interpretation of the disease and led to a conclusion that Alzheimer's disorder is a "fast" disease preventable by therapeutic intervention initiated even late in life and both treatable and potentially curable at the symptomatic stages. 
But why the candidate drugs were so effective in mouse $\mathrm{AD}$ models? To understand this, it is instructive to address the apparent exclusivity of the disease to Homo sapiens. All non-human mammals tested to date accumulate $A \beta$ as they age but they do not develop $\mathrm{AD}$. This is true for small-sized, short-lived animals such as mice, and for large-sized, long-lived non-human mammals such as elephants. In contrast, humans do both. It appears, therefore, that the levels of beta-amyloid sufficient to trigger a cascade of molecular events culminating in $\mathrm{AD}$ can be reached physiologically in Alzheimer's disease patients but not in non-human mammals (or in healthy humans). In terms of the insights formulated above, the $\beta$ APP-independent pathway of production of intraneuronally retained $A \beta$ operates only in humans (and only in disease) but not in animals where $A \beta$ is produced only by $\beta A P P$ proteolysis (the possible reasons for this are discussed below). Moreover, in mouse models of $A D$, production of $A \beta$, both endogenous and from usually multiple transgenes, occurs solely in the $\beta$ APP proteolytic/ secretory pathway.

Within the framework of the notion that $\mathrm{AD}$ symptoms are caused by the intracellular $A \beta$ accumulated to and maintained at sufficient levels, it is very clear why candidate AD drugs worked well in animals models, where $A \beta$ is produced/secreted solely via $\beta A P P$ proteolysis, but not in humans, where the $\beta A P P-i n d e p e n d e n t$ pathway of generation of intraneuronally retained $A \beta$ is also operational in Alzheimer's disease. As discussed in more details below, $A \beta$ generated in the $\beta$ APP proteolytic/secretory pathway accumulates intracellularly by two mechanisms, the retention of a small fraction of the $\beta A P P$-derived $A \beta$ output and the cellular uptake of secreted $A \beta$. BACE inhibitors impact both mechanisms: Less $A \beta$ is produced, less is retained; less $A \beta$ is secreted, less is taken up. Antibodies block the cellular uptake of secreted $A \beta$ and, probably through a feedback mechanism, shift $A \beta$ retention/ secretion equilibrium toward the latter. In animal models, with both types of drugs, levels of intracellular $A \beta$ and its deleterious effects are reduced, thus producing powerful therapeutic outcomes. In $\mathrm{AD}$ in humans, the same processes also occur. But because in Alzheimer's disease the bulk of intraneuronal $A \beta$ is produced (and retained) in the $\beta$ APP-independent manner, drugs targeting $\beta$ APP proteolysis (BACE inhibitors) or extracellular $\mathrm{A} \beta$ ( $\mathrm{A} \beta$ antibodies) have little or no effect.

In the context of the present discussion, we would like to comment on the recent, accelerated and highly controversial, FDA approval of a presumed AD "drug". In June 2021, FDA approved a Biogen/Eisai monoclonal antibody against betaamyloid, aducanumab. It was approved despite the lack of efficacy in human clinical trials and contrary to the recommendation of the Scientific Advisory Board (SAB). The approval of aducanumab prompted resignation in protest of several $\mathrm{SAB}$ members and caused numerous physicians and physicians' organizations to declare that they would not prescribe aducanumab to Alzheimer's patients. The aducanumab approval appears to be a lobbying triumph that, regretfully, also casts doubt on the competence of political bureaucracy to regulate matters of scientific nature. The Office of Inspector General, US Department of Health, was asked to investigate the aducanumab approval process. The investigation is ongoing.

Potentially Pivotal Role of The AUG Encoding Met671 Of Human $\beta A P P$ In $\beta A P P$ -

\section{Independent Generation Of Beta-Amyloid In Alzheimer's Disease}

In light of the above discussion, the $\beta$ APP-independent pathway of $\mathrm{A} \beta$ generation emerges as the core of Alzheimer's disease etiology. Accordingly, the elucidation of the process by which $A \beta$ is produced independently of $\beta A P P$ is pivotal for the understanding of $\mathrm{AD}$, for the construction of the appropriate models of the disease, and for the development of effective drugs. A possible mechanism for just such a process was, in fact, proposed early on in the "molecular" era of $\mathrm{AD}$ research. The human gene encoding amyloid precursor protein, of which beta-amyloid is a part, was identified, cloned and sequenced in 1987 [2-4]. The same year, Breimer and Denny [5] noticed that in this gene, the segment encoding $A \beta$ portion of the precursor protein (starting with the GAU codon encoding Asp672 of the $\beta A P P$ ) is preceded contiguously by the AUG codon normally encoding Met671 of the $\beta$ APP. They also noticed that the AUG codon in question is situated within an optimal translation initiation context, an A in position (-3) and a $\mathrm{G}$ in position (+4) relative to the A of the AUG, and that of all 20 methionine-encoding AUG codons in the human $\beta A P P$ mRNA, including the AUG encoding Met that initiates $\beta A P P$ translation, the AUG encoding Met671 of the $\beta A P P$ is the only one situated within an optimal translation initiation context. Breimer and Denny argued that such a propitious localization of the AUG encoding Met671 of the $\beta$ APP may reflect the underlying physiological function, and suggested that this function could be the internal initiation of translation of $\beta$ APP mRNA, a process which, they proposed, is induced and operates in Alzheimer's disease. They further suggested that in such a case, translation initiated at the AUG in question would produce, after cotranslational removal of the $\mathrm{N}$-terminal methionine by $\mathrm{N}$-terminal methionine aminopeptidases 1 and 2 (MAP1 and MAP2), the C99 fragment of $\beta$ APP, which would be subsequently processed into $\mathrm{A} \beta$ by gamma-secretase cleavage of C99. Importantly, in such a process, C99 and, subsequently, A $\beta$ derived from it would be produced independently of $\beta$ APP, but, in Breimer and Denny's interpretation, would be indistinguishable from $\mathrm{C} 99$ and $\mathrm{A} \beta$ generated in the $\beta$ APP proteolytic pathway [5] (the latter point is further discussed and contested below).

The possibility of internal initiation of translation of $\beta A P P$ mRNA, at the AUG codon normally encoding Met671 of $\beta$ APP, proposed by Breimer and Denny [5], was eventually ruled out by multiple studies [6,7] (please see the additional relevant discussion below). However, there is another possibility of utilization of the AUG codon, normally encoding Met671 of $\beta$ APP, in conventional initiation of translation, namely the generation of severely 5 -truncated $\beta A P P$ mRNA where the AUG codon in question is the first functional translation initiation codon. Several potential mechanisms of generation of such mRNA molecules are described below. One such mechanism is asymmetric RNA-dependent human $\beta A P P$ mRNA amplification, a variant of the Two-Tier mRNA amplification pathway implicated in overproduction of specific mammalian mRNA species [8]. Because of the plausibility of its occurrence in $\mathrm{AD}$-affected neurons, this mechanism is of special interest in terms of its potential operation in Alzheimer's disease. Indeed, as detailed below, human $\beta A P P$ mRNA appears to be an eligible substrate in the mammalian mRNA amplification pathway, and processes capable of activating this pathway are known to be operational in $\mathrm{AD}$-affected cells. 


\section{Mammalian RNA-Dependent mRNA Amplification: Tier One, The Chimeric Pathway \\ Origins of the field}

The initial indications of mammalian RNA-dependent mRNA amplification were obtained in studies of the kinetics of globin mRNA synthesis in differentiating erythroid cells, which strongly suggested the occurrence of cytoplasmic de novo production of globin mRNA [9]. In these experiments, unexpectedly, in very short labeling pulses, the bulk of radioactivity incorporated into globin mRNA and anticipated to be localized solely in the nuclei, was accounted for in the cytoplasm [9]. The labeled molecules appeared to be of a genuine cytoplasmic origin. Indeed, in the nuclei, globin RNA is synthesized in the form of a $15 \mathrm{~S}$ precursor, which is processed to its mature $9 \mathrm{~S}$ size with a half-life of about 10 minutes. Following the seconds-long pulse, only mature size radioactive globin RNA was seen in the cytoplasm and only precursor-size molecules in the nuclei [9]. In such pulse-labeling, cytoplasmic globin RNA was labeled uniformly and not by the end addition to preexisting molecules, consistent with de novo synthesis utilizing mature cytoplasmic globin mRNA as the initial template. Moreover, a high concentration of actinomycin D completely inhibited incorporation into nuclear globin RNA but had little effect on globin RNA labeling in the cytoplasm, suggesting the involvement of a distinct enzymatic activity in cytoplasmic RNA synthesis [9]. Two additional lines of evidence suggested a possible involvement of mammalian RNA-dependent RNA polymerase, RdRp, an enzymatic activity previously detected in and isolated from rabbit reticulocytes [10]. One was the detection of globin antisense RNA, of a size comparable with globin mRNA and apparently containing a 5 ' poly(U) segment, presumably a complement of the 3'-terminal poly(A) of globin mRNA. Another was the detection of the RdRp enzymatic activity in erythroid cells, at the levels greatly exceeding those seen in undifferentiated control cells [9].

Findings described above were substantiated more directly in studies with cytoplasts, i.e. erythroid cells enucleated in vitro by mechanical manipulations [11]. Utilization of cytoplast preparations, shown to be free of contaminating nucleated cells, allowed definitive corroboration of the occurrence of cytoplasmic synthesis of both positive- and negative-strand globin RNA. Cytoplasts were further selectively permeabilized, enabling the use of substances that do not readily cross the plasma membrane. By using mercury-substituted CTP as a substrate, it proved possible to physically separate newly synthesized globin RNA by adsorption to thiol-agarose, and show that the cytoplasmic incorporation of radioactive precursors into both sense and antisense globin RNA constituted a de novo synthesis. The system also allowed experiments establishing that synthesis of cytoplasmic globin RNA required the presence of $\mathrm{Mg}^{++}$, was inhibited by $\mathrm{Mn}^{++}$and showed no response to $\mathrm{Zn}^{++}$, in contrast to viral RNA replicases. Cytoplasmic globin RNA synthesis was resistant to actinomycin $\mathrm{D}$, alpha-amanitin and rifampicin, but inhibited by the rifampicin derivative AF/ABDP. The results also established that a de novo DNA synthesis was not involved in the observed phenomenon: Synthesis of RNA occurred in permeabilized cytoplasts not only without the addition of dNTPs but also when ddNTP was added [11].
The apparent presence in antisense globin RNA of a $5^{\prime}$ poly(U) region, presumably a transcript of the 3'-terminal poly(A) segment of mRNA, indicated that synthesis of the antisense strand initiates at the poly(A) of its mRNA template. The precedence for initiation of antisense RNA synthesis within the poly(A) is well established for some viral systems where it is primed by a uridylated protein; in mammalian systems, different mechanisms for initiation of antisense RNA synthesis could be involved. A mechanism for the second stage of RNA-dependent globin mRNA synthesis, the initiation of synthesis of sense globin RNA on the antisense RNA strand template, was suggested in a study of the generation of a largely double-stranded murine beta-globin cDNA by RNAdependent DNA polymerase ( $\mathrm{RdDp}$ ), a process that involves formation of covalently linked antisense and sense strands in a hairpin configuration [12]. This study examined a sequence of molecular events, particularly the self-priming, that initiates synthesis of the sense strand. It was shown that upon completion of reverse transcription of globin mRNA and the removal of the RNA template by RNase $\mathrm{H}$ activity associated with $\mathrm{RdDp}$, the 3 ' terminus of the antisense strand snaps back to form a stable double-stranded self-priming structure which is extended by $\mathrm{RdDp}$ to generate the sense strand. The self-priming event is enabled by strong complementarity of 14-nucleotide-long 3'-terminal segment of the antisense strand (3'-Terminal Complementary Element, TCE) with an internal segment of the same molecule (Internal Complementary Element, ICE) corresponding to a portion of the 5'untranslated region, 5'UTR, of mRNA located just upstream of the translation start site. Surprisingly and informatively, the strong complementarity between the terminal and the internal complementary elements occurs within the antisense but not the sense strand. This is because A:C mismatches on the sense strand correspond to stable $\mathrm{T} / \mathrm{U}: \mathrm{G}$ base pairs on the antisense strand. The self-priming-enabling complementarity within the betaglobin antisense RNA strand was shown to be highly preserved throughout a vast evolutionary distance, from marsupials to humans [12], with conservation of not only the occurrence of complementary elements but also their positions within the antisense RNA segment corresponding to the 5'UTR of mRNA; although nucleotide sequences diverged substantially during evolution, the complementary relationship of the 3' terminal and the internal elements of the antisense RNA, as well as the position of the internal element within a segment corresponding to the 5'UTR of mRNA, remained preserved, strongly suggesting that their functionality is physiologically relevant.

If cellular RdRp activity utilizes the same self-priming arrangement as RdDp to extend the antisense globin RNA strand into a sense-orientation molecule, the resulting hairpin-structured RNA would have to be cleaved to separate the globin RNA strands. Where the cleavage may occur within the hairpin structure, was indicated by a detailed characterization of the antisense globin RNA in mouse erythroid tissues [13]. This study made use of a multistep procedure in which a molecular tag is attached to cellular RNA by ligation with a defined ribooligonucleotide. The act of ligation preserves the termini of RNA molecules, which become the junctions between cellular RNA and ligated ribooligonucleotide. It also unambiguously preserves the identity of cellular RNA as a sense or antisense molecule through all subsequent manipulations culminating with nucleotide sequencing of tag-containing globin clones $[13,14]$. This approach resulted in identification 
and characterization of antisense beta-globin RNA molecules in mouse erythroid tissues [13]. The antisense RNA was shown to be fully complementary to spliced globin mRNA, indicative of the template/transcript relationship. At the 5' end, antisense globin RNA terminates with a uridylate stretch, reflecting the presence of the poly(A) at the 3' end of the sense globin RNA and indicating that synthesis of the former initiates at the 3 '-terminal poly(A) region of the latter. With respect to the structure of their 3 ' termini, the detected antisense globin RNA could be divided into two classes of interest. One class represented a minor population and consisted of full-size antisense molecules corresponding precisely to globin mRNA. The other class represented the major globin antisense RNA population and consisted of 3'-truncated molecules. The truncation was not random; 3 '-truncated antisense RNA molecules lacked predominantly fourteen 3'-terminal nucleotides, the same 14-nucleotide segment that constitutes the 3 '-terminal complementary element required for the formation of globin antisense RNA self-priming structure.

A compendium of observations and considerations described above suggested a model for RNA-dependent amplification of mammalian mRNA [13]. In this postulated process, RdRp transcribes mature spliced cytoplasmic mRNA. Such transcription initiates at the 3' poly(A) region of mRNA and produces the antisense RNA containing poly $(\mathrm{U})$ at the $5^{\prime}$ end and terminating at the 3 ' end with a complement of the 5' terminus of the mRNA molecule. The subsequent transcription of the antisense RNA was postulated to occur via self-priming and extension of its 3 ' terminus. Cleavage within a single-stranded loop of the resulting hairpin-structured molecule and the unwinding of a doublestranded structure would then produce a 5 '-truncated sense strand RNA terminating with the poly(A) at the 3' end and containing an antisense segment at the 5' terminus, and a 3'-truncated antisense RNA strand. If self-priming occurs within a segment corresponding to the 5'UTR of mRNA, the resulting sense RNA component would contain the entire protein coding information of the original mRNA and could be translated into a polypeptide indistinguishable from the conventionally produced protein. In the case of globin mRNA amplification, the observed predominant 14-nucleotide 3'-terminal truncation of the antisense globin RNA strongly suggested that the cleavage occurs mainly at the 3' end of the single-stranded loop.

At the core of the mechanism proposed in [13] and described above is a distinctive extension of the self-primed antisense RNA strand. Therefore, the detection of the major recognizable attribute of this mechanism, an antisense RNA molecule extended into a sense strand in a rigorously predicted and uniquely defined manner, would provide conclusive evidence for the proposed mechanism of the mammalian mRNA amplification process. Two types of postulated RNA molecules would, due to their intrinsic structure, contain such evidential information. One is the largely double-stranded/hairpin-structured chimeric intermediate consisting of a sense and an antisense strands covalently joined in a precisely defined way, a direct precursor of the amplified mRNA end product. The other is the chimeric RNA end product containing a defined antisense RNA fragment joined to a predictably 5'-truncated sense RNA at its 5' terminus. Due to their transient nature, chimeric intermediates could be a rarity, but the chimeric end product could be expected to represent the majority of amplified mRNA sequences. In both cases, the region of interest, an "identifier" of the amplification process, is the junction between the sense and the antisense components. The occurrence of such chimeric sense/antisense junction sequences involving both alpha- and beta-globin chains-encoding mRNAs was, therefore, investigated in murine erythroid tissues using next generation RNA sequencing $[15,16]$. A selection of chimeric fragments detected for both alpha- and beta-globin RNA is presented in Figure 1, together with alpha- and beta-globin genomic reference sequences, conventional globin mRNA sequences, postulated chimeric sequences, and projected folded antisense sequences whose 3' extension would generate predicted chimeric sequences. Two Types of chimeric reads were obtained for both alphaand beta-globin RNA sequences. Type One reads comprised the predicted chimeric junction sequences resulting from the extension of the full-size self-primed antisense RNA. The other Type of reads (two upper reads for alpha-globin and two bottom reads for beta-globin in "Chimeric junctions" panels of Figure 1) contained antisense/sense junction sequences consistent with their origination from a 3 '-truncated antisense RNA retaining a portion of its 3'-terminal complementary element apparently sufficient for the self-priming and extension. Whereas the occurrence of the Type One of chimeric junctions was anticipated, the detection of the second Type was unexpected. Equally unexpected was the observation that the antisense portions of the Type One alphaglobin chimeric junctions uniformly contained at their 3' termini the additional " $\mathrm{C}$ " evidently not encoded in the genome. Most startling was the observation that all detected chimeric junctions appeared to originate from chimeric RNA intermediates; not a single chimeric RNA end product sequence read was identified despite its anticipated domination among globin RNA sequences. As the research progressed, it became apparent that the observed unanticipated features reflect various mechanistic aspects of the mRNA amplification process, which are addressed below.

The observations described above raised a question: Is mammalian RNA-dependent mRNA amplification a special occurrence limited to extreme circumstances of terminal erythroid differentiation or is it a general physiological phenomenon? This question was addressed [17] by testing for the occurrence of RNA-dependent amplification of mRNA encoding extracellular matrix proteins that are abundantly produced throughout the development and homeostasis, arguably the best indicator of the potential range and scope of this phenomenon. As shown in Figure 2, the major identifiers of RNA-dependent amplification of mRNA encoding alpha1, beta1, and gamma1 chains of laminin were indeed detected in mouse tissues producing large quantities of extracellular matrix proteins [17]. Multiple types of chimeric junctions observed for each of three laminin chains-encoding RNAs are consistent with their origin from conventional mRNAs transcribed from normally occurring multiple Transcription Start Sites (TSS) of TATA-less laminin genes. Some of these variations could also be due to the re-use of the antisense components of processed chimeric intermediates and the consequent "chimeric junction shift" discussed below. All unanticipated features of the mRNA amplification process seen with globin RNAs were also observed with laminin RNAs. The results obtained, therefore, established RNA-dependent mRNA amplification as a general physiological phenomenon in mammalian cells. This process, its features, as well as its mechanistic and regulatory aspects, are discussed in details in the following sections below. 


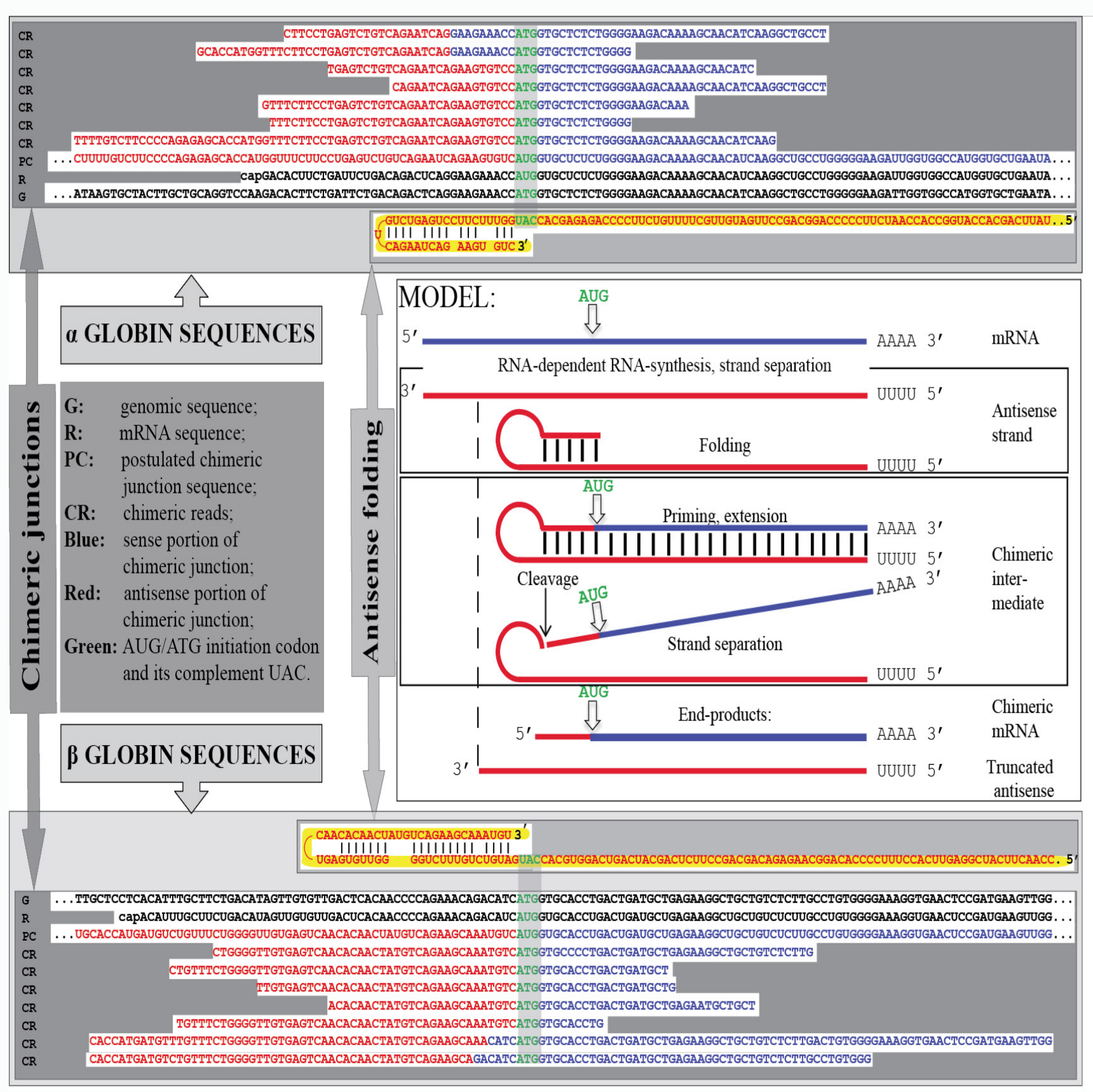

Figure 1: Substantiation of the proposed mechanism for RNA-dependent mRNA amplification: Detection of the chimeric RNA junctions containing globin antisense RNA sequences extending into sense-orientated RNA in a rigorously predicted manner. "Model" panel: Diagrammatic representation of a model for mammalian RNA-dependent mRNA amplification described above; "AUG"- the AUG translation initiation codon. Antisense RNA folding panels (highlighted in yellow, immediately above and below the "Model" panel): Postulated folding/self priming of the antisense globin RNA, preceding its extension into the sense RNA strand. Chimeric junction panels: Selection of sequences containing the antisense RNA extended into the sense orientated RNA exemplifying different categories of the reads obtained (i.e. two upper reads for alpha-globin and two bottom reads for beta-globin consistent with originating from 3'-truncated antisense RNAs; rest of the reads originate from complete antisense RNA). Abbreviations and color-coding: Specified in the center-left portion of the Figure.

\section{Current Understanding of the Chimeric Pathway of Mammalian mRNA Amplification: A Brief Summary}

The chimeric pathway of mammalian RNA-dependent mRNA amplification, as currently understood, is presented diagrammatically in Figure 3 and can be briefly (details in sections below) summarized as follows. The amplification process starts with synthesis of the antisense complement of a conventional, gene-transcribed mRNA by the RdRp complex. It commences with the 5'-terminal poly $(\mathrm{U})$ transcribed from the 3'-terminal poly(A) of a mRNA and produces a complete antisense RNA molecule. A helicase complex then separates strands of the double-stranded sense/antisense RNA structure. Upon separation, the sense RNA molecule becomes available to serve again as a template for the antisense RNA synthesis, whereas the disengaged antisense RNA strand forms a self-priming structure and its 3 ' terminus is extended by RdRp into the sense strand RNA, terminating with the poly(A) 


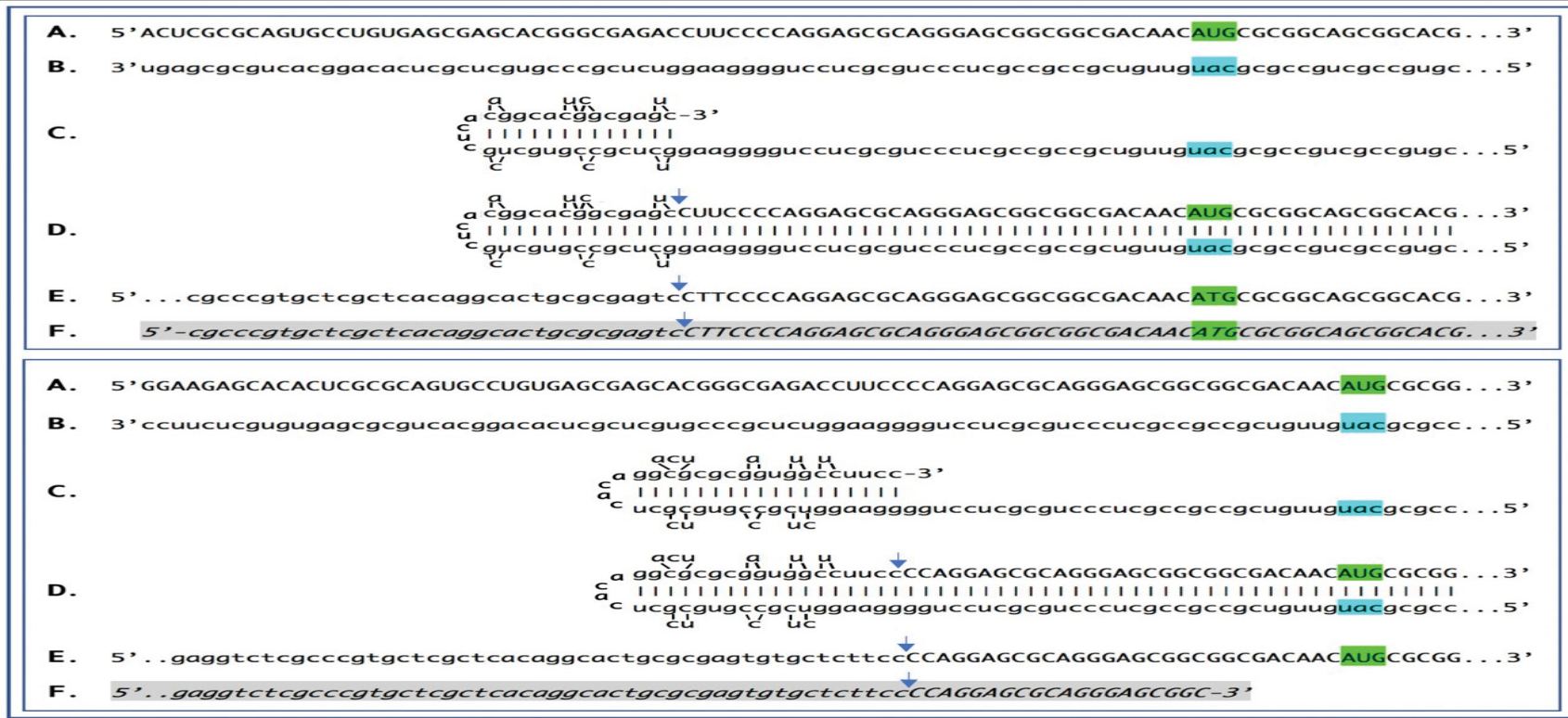

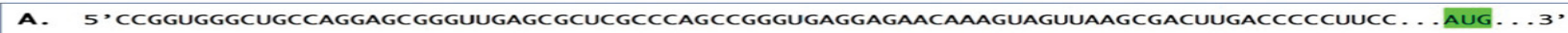

B. 3'ggccaccegacgguccucgcccaacucgcgagcgggucggcccacuccucuuguuncaucaauucgcugaacuggaggaagg. . . uac. . .5,

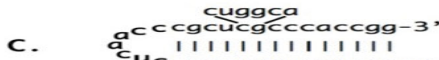

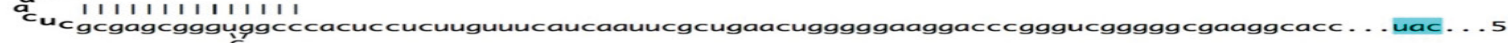

D.

giuggca

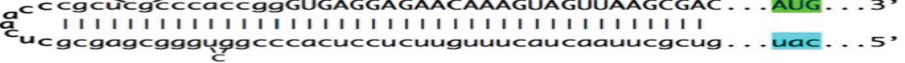

E. 5 , ..ttctcctcacccggctgggcgagcgctcaaccegctcctggcagcccaccgăGTGAGGAGAACAAAGTAGTTAAGCGAC. . AUG. . 3,

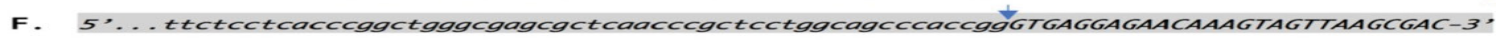

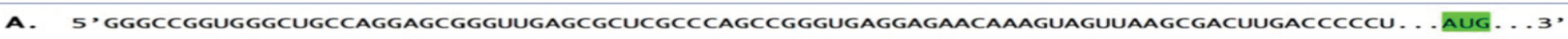

B. 3'cceggccaccegacgguccucgcccaacucgcgagcgggucggcccacuccucuuguuucaucaauucgcugaacuggggga . . . uac... .5,

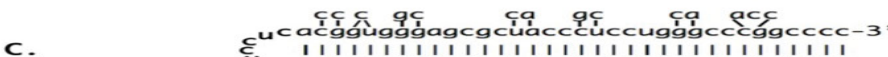

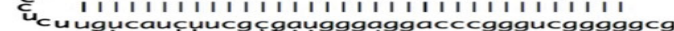
ưं

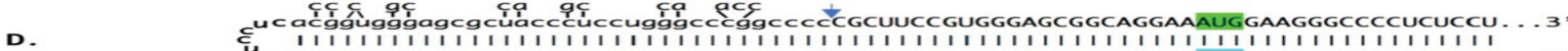

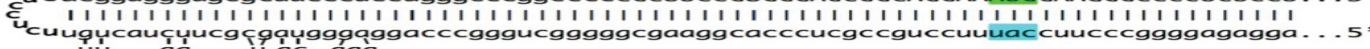

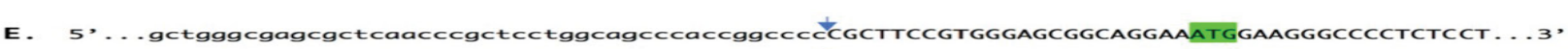

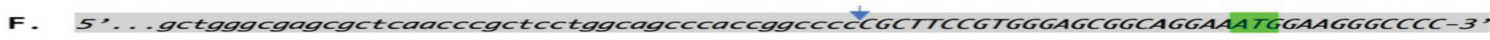

A. 5' CGGCGCACAGUGCAGGCUGCGCACCGgGAGGUAGCGGAGGCAGCGCGAUCUUGGCUCGGACGCCCACCCAUCGGCUCUGCGU... AUG. 3,

B. 3'gcegcgugucacguccgacgcguggcccuccaucgccuccgucgcgcuagaaccgagccugcggguggguagccgagacgca . . .uac. . . 5 ,

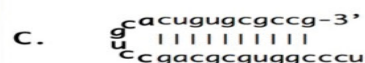

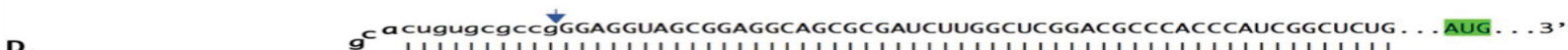

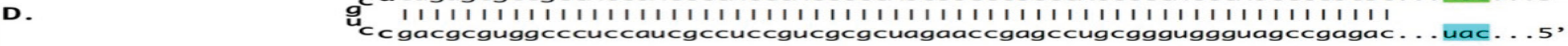

E. 5, ... cggtgegCagCCtgCaCtgtgCgCCğ̈GGAGTAGCGGAGGCAGCGCGATCTTGGCTCGGACGCCCACCCATCGGCTCTGCGTCCG . . 3,

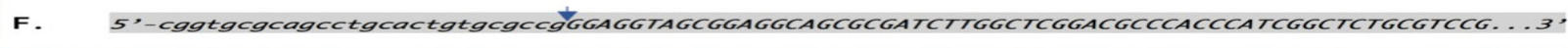

A. 5' GCGCGCUCUCGAGUGCGCGCUCGGAAGUCGGGGGUCGGCGCACAGUGCAGGCUGCGCACCGGGAGGUAGCGGAGGCAGCGCG .. . AUG . . 3'

B. 3'cgcgcgagagcucacgcgcgagccuucagcccecagcegcgugucacguccgacgcguggcecuccaucgccuccgucgcgc... uac. . .

C. $\quad$ gágegcgc-3

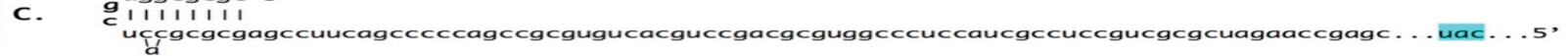

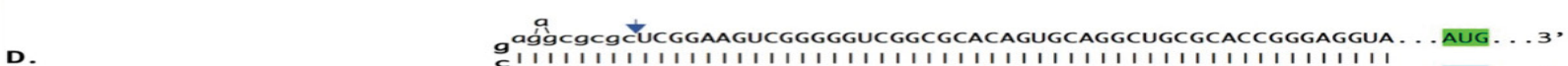

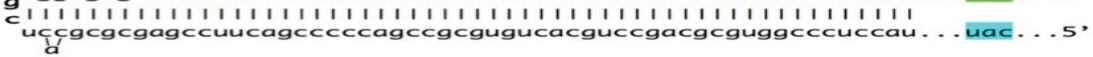

E. 5 . . . ccgacttccgagcgcgcactcgagagcgcg đ̇́TCGGAAGTCGGGGGTCGGCGCGCAGTGCAGGCTGCGCACCGGGAGGTAGCGGAG . . 3 ,

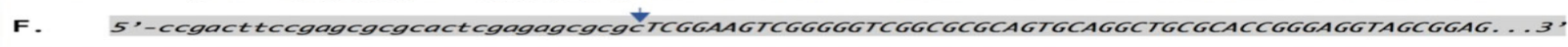


Figure 2: Sequences of chimeric junctions containing antisense and sense segments of laminin $\alpha 1$ (top panel), $\beta 1$ (middle panel) and $\gamma 1$ (bottom panel) mRNAs and the projected pathways of their generation. Uppercase letters: Nucleotide sequences of the sense RNA strands; lowercase letters: Nucleotide sequences of the antisense RNA strands. Highlighted in green: "AUG" translation initiation codons on the sense RNA strands; highlighted in blue: "uac" complements of translation initiation codons on the antisense RNA strands. In italics and highlighted in grey: Detected chimeric fragments. Blue arrows: Positions of antisense/ sense RNA junction. The top and bottom subpanels of each panel depict amplification of mRNA molecules presumably transcribed from different TSSs; note that selfpriming positions and, consequently, chimeric junction sequences shown in two subpanels of each panel are different. A: 5'-terminal regions of conventional mRNA. B: Antisense complements of the 5'-terminal regions of conventional mRNA. C: Folding of the antisense RNA strands into self-priming configurations. D: Extension of selfprimed antisense RNA strands into sense-oriented RNA sequences. E: Projected chimeric junction sequences. F: Detected chimeric junction sequences. Note: Selfpriming events occur within segments of the antisense RNA strands corresponding to 5'UTR of mRNAs, thus preserving the coding capacity of the amplified mRNAs.

segment at the 3' end. The resulting hairpin structure is resolved by a helicase complex invoked above, starting at the linear end of the double-stranded region; upon reaching the single-stranded loop of the hairpin structure, the helicase, or an associated activity, cleaves the RNA molecule at the 3' end of the loop. This generates two end products of the chimeric mRNA amplification pathway: The antisense RNA truncated at the 3' end and the chimeric RNA molecule consisting of a $5^{\prime}$-truncated sense RNA and a covalently attached, in a 5' to 3' orientation, segment of the antisense RNA (its cleaved-off 3'-terminal portion). Thus, the manner of generation and processing of the chimeric intermediate leads to both the loss and the acquisition in the resulting chimeric RNA end product: The loss of a portion of conventionally produced mRNA and the acquisition, via transfer of a segment of the antisense RNA to its sense RNA counterpart (rendering the former 3'-truncated and the latter "chimeric"), of a component not present in a conventional gene-transcribed mRNA progenitor of the amplification process. The future fate of the 3'-truncated antisense RNA end product can be remarkable and is discussed in the "Tier Two" sections below. The chimeric RNA end product, on the other hand, constitutes the "functional" outcome of the chimeric pathway. Its observed cellular levels reflect the potency of the amplification process: At the peak of the erythroid differentiation, for example, the amount of globin mRNA produced in the chimeric amplification pathway is about 1500 -fold higher than the amount of conventionally generated globin mRNA in the same cells $[15,16]$. Potentially, the chimeric RNA end product can be translated into a polypeptide $[15,16]$. The possible translational outcomes of the chimeric pathway of mammalian mRNA amplification, however, are neither uniform nor necessarily identical to that attained with a conventional mRNA progenitor; they are rather multiple, conceivably drastically different, and can be grouped into the four Types as follows.

Type I of the Chimeric Pathway of Mammalian mRNA Amplification Preserves Complete Protein-Coding Information of Conventional, Gene-Transcribed, mRNA Progenitor

Depending on the structure and nucleotide sequence of the initial mRNA amplification progenitor (or, more precisely, of its antisense RNA complement), translational outcomes of the chimeric pathway of mammalian mRNA amplification can vary drastically and cover the entire spectrum from a polypeptide identical to one encoded by a corresponding conventionally produced mRNA progenitor, to only a C-terminal fragment of the latter, to a polypeptide non-contiguously encoded in the genome, to a completely unrelated polypeptide and even to an abortive translation or translational incompetence. Which variant of the chimeric pathway will occur with a particular mRNA species is determined primarily by the site of the self-primed extension of the 3' terminus of the antisense RNA (Figure 3, Step 3). This, in turn, is defined by the position of the internal complementary element, ICE, within the antisense RNA molecule. This is because the 3' terminal complementary element, TCE is, by definition, always 3'-terminal, whereas the ICE can be potentially located anywhere within the antisense RNA. In the chimeric pathway Type I, depicted in Figure 3, the ICE is located within a segment of the antisense RNA corresponding to the 5'UTR of conventionally produced mRNA molecule. Therefore, the sense-oriented RNA, produced by the extension of the TCE, contains a portion of the 5'UTR and the entire coding region of the conventionally produced mRNA utilized as the initial amplification template (Figure 3, Step 4). The translational outcome in this case is a polypeptide identical to one translated from a conventional gene-transcribed mRNA progenitor. As seen in Figures 1 and 2, this Type of chimeric pathway is characteristic for amplification of mRNAs encoding alpha- and beta-globin chains $[15,16]$ and all three chains (alpha1, beta1 and gamma1) of laminin [17]. Moreover, in the globin case, the translational product of the amplified globin mRNA was shown to be indeed indistinguishable from conventionally produced globin polypeptides $[15,16]$. This Type of the chimeric pathway is expected to take place in most physiologically occurring cases of mammalian RNA-dependent mRNA amplification.

\section{Type II of the Chimeric Pathway of Mammalian mRNA Amplification Reduces or Changes the Protein- Encoding Information Content of Conventional mRNA Progenitor}

In the chimeric mRNA amplification pathway, the position of the TCE element within the antisense RNA molecule is fixed: It is always strictly 3 '-terminal. In contrast, the intramolecular location of the internal ICE element is variable, and potentially it can be positioned within a segment of the antisense RNA strand corresponding to the coding portion of an mRNA, a scenario diagrammed in steps 3' through 7' of the bottom panel of Figure 4. In this scenario, the chimeric RNA end product consists of a 3'-terminal segment of the antisense strand (the TCE or its fraction) attached, in a 5' to $3^{\prime}$ orientation, to a portion of a conventional mRNA amplification progenitor with a 5 '-truncated coding region. In such a case, the translational outcome would be decided by the position of the first functional (capable of initiation of translation) AUG or another translation initiation-competent codon. If it were in-frame with the protein-encoding information content of conventional mRNA, translation would result in the C-terminal fragment, CTF, of a conventional mRNA-encoded polypeptide. This variant of the chimeric RNA-dependent mRNA amplification pathway would be asymmetric. Indeed, only one end, a 3'-terminal portion of the coding region continued into the 3'UTR, of conventional mRNA would be amplified, and its translation would potentially produce only one end of a corresponding conventional mRNA-encoded polypeptide, its C-terminal fragment, CTF. As discussed below, such asymmetric amplification of beta-Amyloid Precursor Protein ( $\beta$ APP) mRNA appears to be the source of beta-amyloid overproduction in Alzheimer's disease. A CTF of a conventionally encoded protein is 


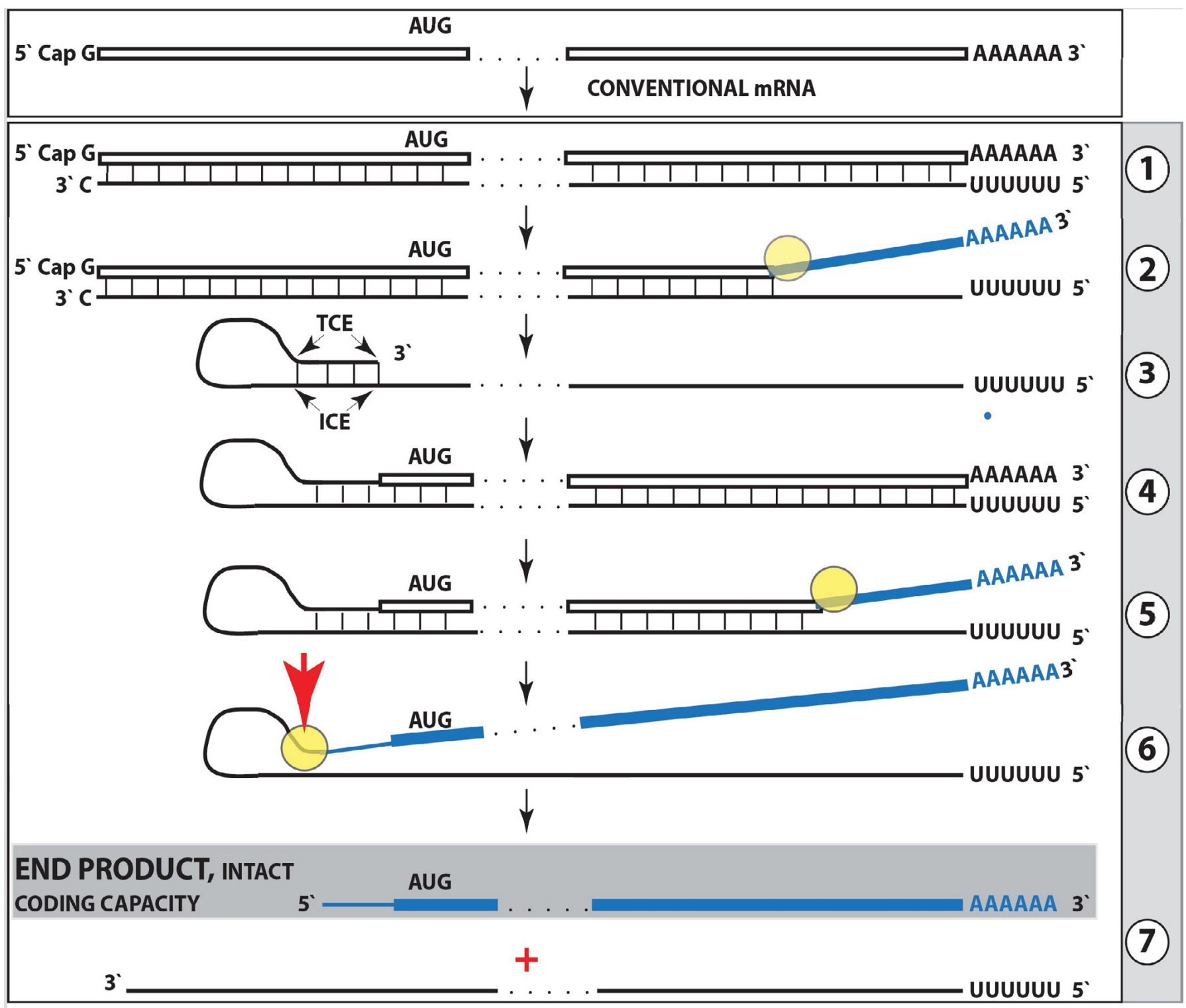

Figure 3: Projected stages of the chimeric Tier of mammalian RNA-dependent mRNA amplification. Top panel: Conventional, genome-transcribed mRNA molecule. Bottom panel: Projected stages of antisense RNA-mediated mRNA amplification. Boxed line: Sense RNA. Single line: Antisense RNA. "AUG": Functional translation initiation codon (could be other than "AUG"). "TCE": 3'-terminal complementary element; "ICE": Internal complementary element, both on the antisense RNA strand. Yellow circle: Helicase/modifying activity complex. Blue lines (both single and boxed): RNA strand, modified and separated from its complement by a helicase complex. Red arrowhead: Position of the cleavage of the chimeric intermediate. Step 1: Synthesis of the antisense strand. Note that, apparently, it is initiated within the poly $(\mathrm{A})$ segment rather than at its end; step 2: Strand separation. Note that it can commence while the poly(U)-containing antisense RNA is still nascent; step 3: Folding of antisense RNA strand into self-priming configuration; step 4: Extension of self-primed antisense RNA; step 5: Strand separation; step 6: Cleavage of the chimeric intermediate; stage 7: End-products of amplification, the 3'- truncated antisense RNA and the chimeric RNA containing a 3'-terminal portion of mRNA and covalently joined cleaved-off 3'-terminal segment of the antisense RNA. Details in "Mechanistic Aspects" sections below.

not the only possible outcome of this Type of chimeric pathway. If the first functional translation initiation codon is not in-frame with the conventional protein-coding information content but precedes in-frame an open reading frame, ORF, an unrelated polypeptide would result. If there were no ORF, an abortive translation would ensue and if a functional translation initiation codon were lacking, the result would be the translational incompetence.

Type III of the Chimeric Pathway of Mammalian mRNA Amplification Results in RNA Containing Protein-Coding Information Non-Contiguously Encoded in the Genome The preceding sections considered RNA-dependent mRNA amplification scenarios where protein-encoding information is contained only in the sense RNA strand. In these scenarios, the chimeric RNA end product either retains the entire coding information of a conventional mRNA, and is translated from the original translation initiation codon, or lacks the 5 -portion of the coding region of a conventionally produced mRNA. In case of the latter, its translation depends either on the availability of a functional in-frame translation initiation codon within the remaining portion of the coding region or, if it is out-of-frame, on the presence of a downstream ORF unrelated to the original information content. However, there is also another possibility. If the TCE of the antisense RNA contains a functional translation initiation codon in the 5' to 3' 


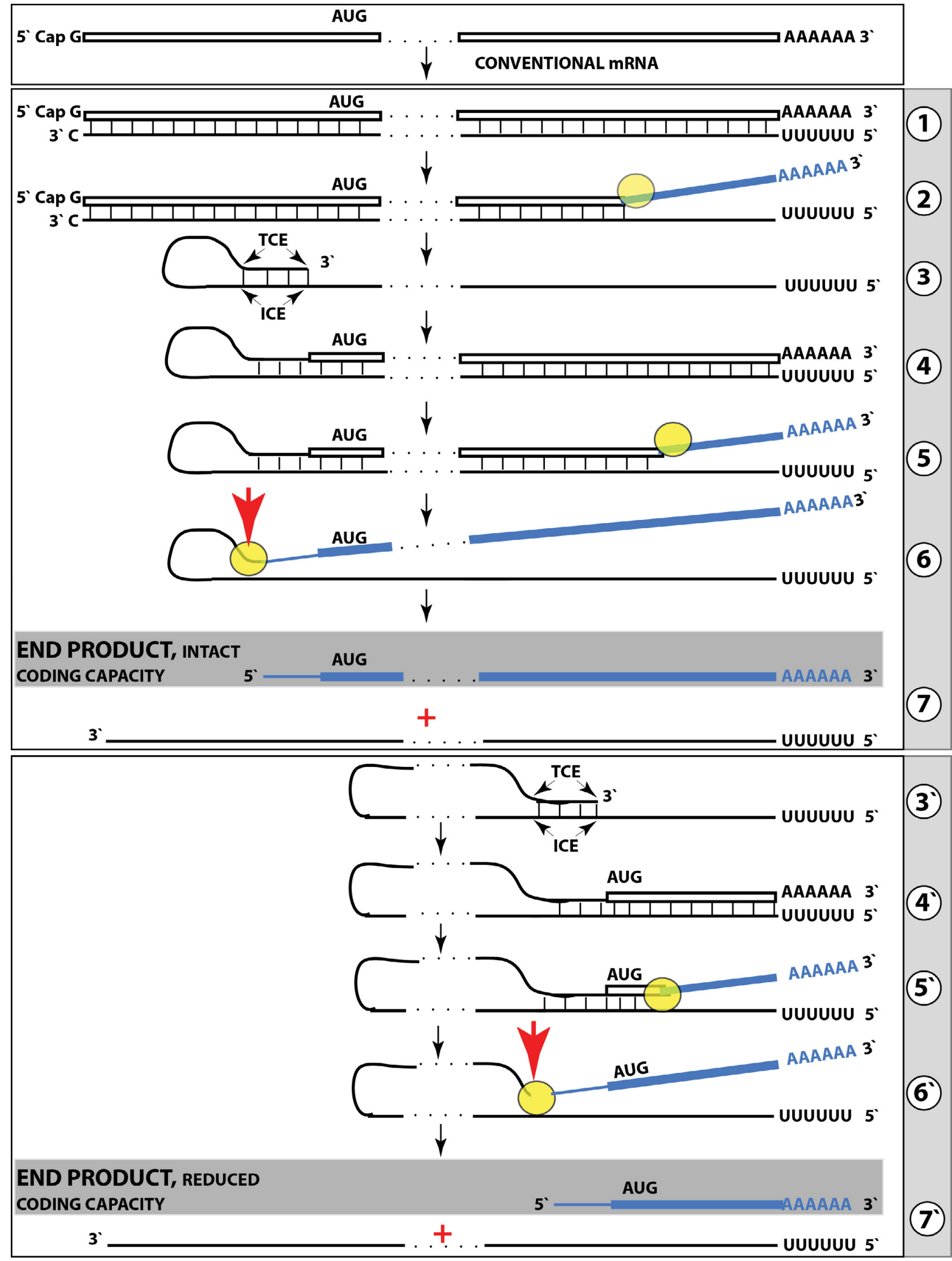


Figure 4: Mammalian RNA-dependent mRNA amplification can result in a chimeric RNAend product encoding C-terminal fragment of a corresponding conventional polypeptide. Boxed line: Sense strand RNA. Single line: Antisense strand RNA. "AUG": Functional translation initiation codon (could be other than AUG). "TCE": 3'-terminal complementary element; "ICE": internal complementary element, both on the antisense RNA strand. Yellow circle: Helicase/ modifying activity complex. Blue lines (both single and boxed): RNA strands, modified and separated from their complements by a helicase complex. Red arrows: Positions of the cleavage of the chimeric intermediate. Step 1: Synthesis of the antisense strand, note that, apparently, it is initiated within the poly(A) segment rather than at its end; step 2: Strand separation, note that it can commence while the poly(U)-containing antisense RNA is still nascent; step 3: Folding of antisense strand into self-priming configuration; step 4: Extension of self-primed antisense RNA; step 5: Strand separation; step 6: Cleavage of the chimeric intermediate; step 7:End-products of RNA amplification. Steps 3'-7' correspond to steps 3-7. Top panel: Conventional, genome-transcribed mRNA molecule. Middle panel: Projected stages of RNA-dependent mRNA amplification. "ICE" is located within a segment of antisense RNA corresponding to the 5'UTR of conventional mRNA; the chimeric RNA end product contains the entire coding content of conventional mRNA. Bottom panel: "ICE" is located within a segment of antisense RNA corresponding to the coding region of conventional mRNA. The amplified chimeric end product contains a 5'-truncated coding region of conventional mRNA. The translational outcome is decided by the position of the first functional translation initiation codon; if in-frame, with the protein-encoding information content of conventional mRNA, translation would result in the C-terminal fragment, CTF, of aconventionally encoded polypeptide; if not-in-frame, multiple potential outcomes, described in text, ensue.

orientation, and if this translation initiation sequence was retained in the chimeric RNA end product after the cleavage of the chimeric intermediate, translation would be initiated from the initiation codon within the antisense portion of the chimeric RNA end product. In such a case, if the folding/self-priming of the antisense RNA occurs within its segment corresponding to the 5'UTR of conventional mRNA, and if the initiation codon within the antisense portion of the chimeric RNA end product is in-frame with the mRNA coding sequence and there are no intervening in-frame stop codons, translation would result in a chimeric protein. It will contain, in its C-portion, the entire polypeptide encoded by a conventional mRNA progenitor, enhanced at its $\mathrm{N}$-end by additional amino acid residues encoded by the antisense portion of the chimeric RNA end product and by a segment of the 5'UTR of conventional mRNA. Thus, interestingly, the proteinencoding information content of the chimeric RNA end product of amplification would be non-contiguously encoded in the genome, as shown in the top panel of Figure 5.

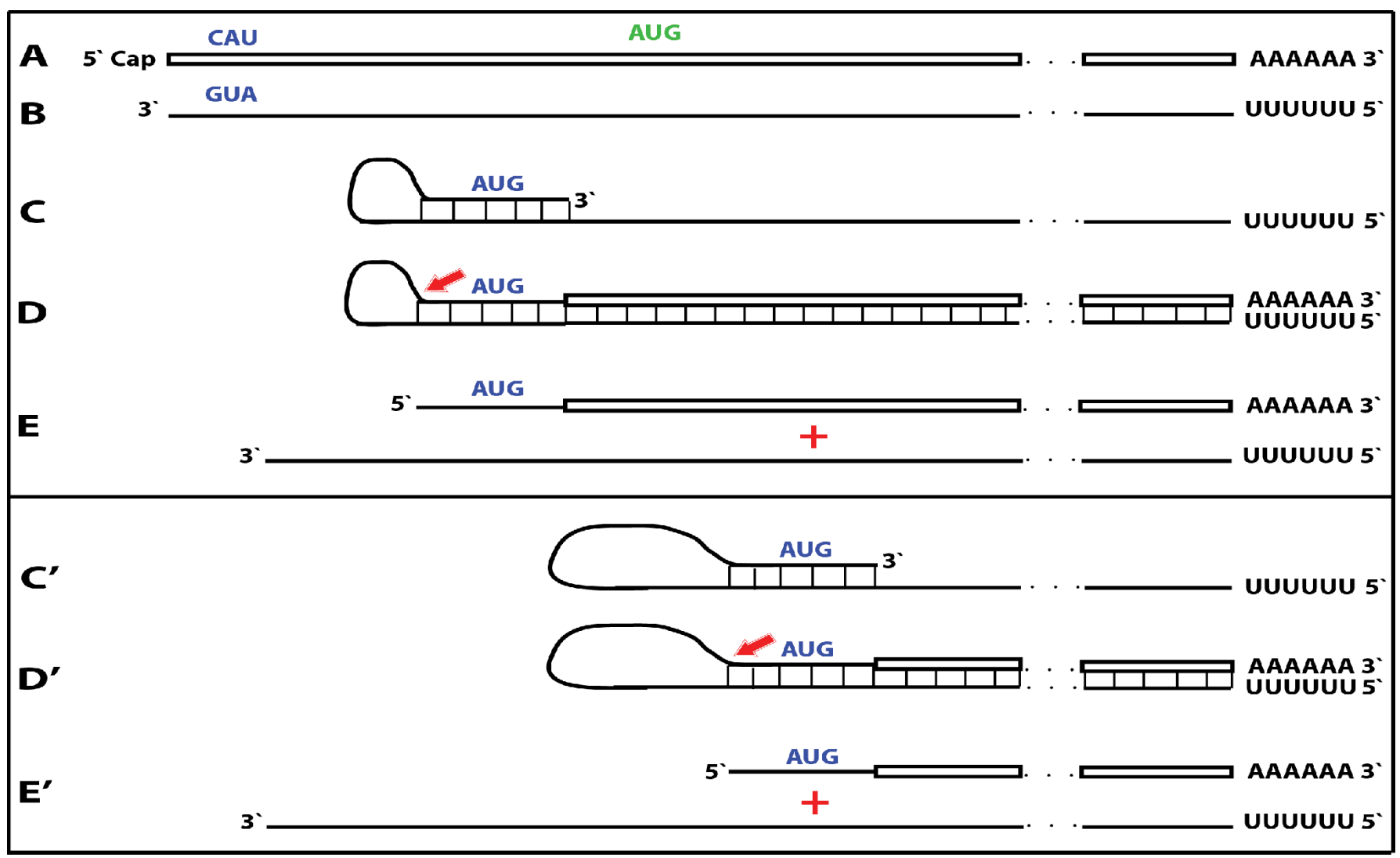

Figure 5: Mammalian RNA-dependent mRNA amplification may enhance protein-encoding information content of a conventional mRNA and generate polypeptides non-contiguously encoded in the genome. Boxed line: Sense strand RNA. Single line: Antisense strand RNA. "AUG" (in green): Translation initiation codon of conventional mRNA progenitor. "AUG" (in blue): Antisense RNA-originated functional translation initiation codon (could be other than AUG). 5'-CAU-3' on the sense RNA: Complement of the 5'-AUG-3' (both in blue) on the antisense RNA. Red arrow: Position of the cleavage of the chimeric intermediate. A: Conventional mRNA; the "AUG" (in green) denotes translation initiation codon. B: Antisense complement of conventional mRNA; 3'-GUA-5' (in blue) is the 5'-AUG-3'. C: Folding of the antisense strand into self-priming configuration. D: Extension of self-primed antisense strand into sense-oriented sequence followed by strand separation and cleavage (red arrow) of the chimeric intermediate. E: The chimeric RNA and 3'-truncated antisense RNA end products of RNA-dependent mRNA amplification. Note that translation of the chimeric end product starts from the antisense RNA-supplied initiation codon (“AUG”, in blue) and produces a chimeric polypeptide non-contiguously encoded in the genome. Steps C', D', and E' correspond to C, D, and E. Top panel: Antisense RNA folding/self priming occurs within its segment corresponding to the 5'UTR of conventional mRNA. Bottom panel: Antisense RNA folding/self priming occurs within its segment corresponding to the coding region of conventional mRNA. Translational outcomes are discussed in the text. 
If, however, the folding/self-priming of the antisense RNA strand occurs within its segment corresponding to the coding region of conventional mRNA, and if the initiation codon within the antisense portion of the chimeric RNA end product is inframe with the mRNA coding sequence, the translational outcome would be a CTF of the conventional polypeptide, enhanced at its $\mathrm{N}$-end by additional amino acid residues encoded by the antisense portion of the chimeric RNA end product, also a chimeric protein non-contiguously encoded in the genome. This variant of the chimeric pathway Type III is illustrated diagrammatically in the lower panel of Figure 5. In both variants shown in Figure 5, if the antisense RNA-derived translation initiation codon were not in-frame with the conventional protein-encoding content, the relevant alternatives described in the preceding section would apply.

Type IV of the Chimeric Pathway of Mammalian mRNA Amplification Activates Dormant Protein-Encoding Information

A scenario considered in this section is one where a conventional gene-transcribed mRNA contains untranslatable protein-encoding information that is expressed as a polypeptide only under specific conditions, for example a particular stress, that activate the RNA-dependent mRNA amplification process, which results, due to the manner of generation and processing of the chimeric intermediate of mRNA amplification, in a translatable chimeric RNA end product. It should be emphasized that regardless of the extent of its physiological occurrence, the understanding of such a scenario is important because it could, and actually does, suggest very useful experimental and bioengineering designs, as described in "Reporter Constructs for the Chimeric Pathway" section below. In this scenario, a conventionally produced RNA encodes a polypeptide but lacks a functional translation initiation codon upstream from and in-frame with the encoded information. Since its potential to produce a protein is present but unrealized under regular circumstances, it can be considered a silent, or "dormant", mRNA. The potential to direct synthesis of a polypeptide can be realized, and a dormant mRNA activated, by the mRNA amplification process. In the chimeric pathway of mRNA amplification, the chimeric RNA end product contains a 5 '-terminal segment contributed by the antisense RNA and not present in the conventional genome-transcribed RNA molecule. If this additional segment contains a functional translation initiation codon, and if this codon is in-frame with the encoded information, the protein-encoding content of a conventional "dormant" mRNA would be activated in the chimeric RNA end product of amplification. The resulting translational outcome would be a chimeric protein consisting of a conventionally encoded polypeptide with the $\mathrm{N}$-end encoded by the antisense RNA. This chimeric protein would be, in similarity to the preceding scenario, also non-contiguously encoded in the genome. The above scenario is diagrammed in Figure 6.

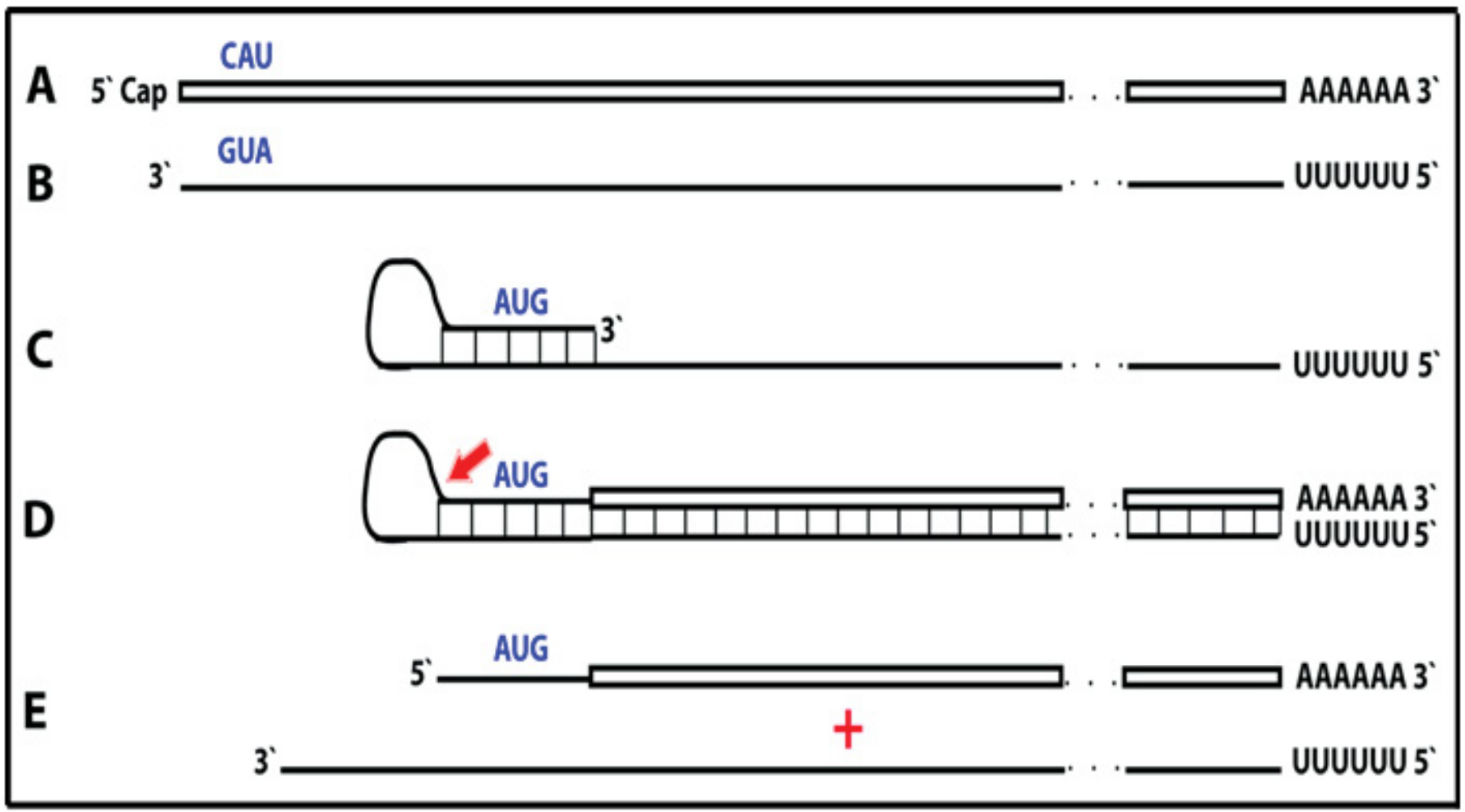

Figure 6: Mammalian RNA-dependent mRNA amplification may activate a dormant protein-encoding information and generate polypeptides noncontiguously encoded in the genome. Boxed line: Sense strand RNA. Single line: Antisense strand RNA. "AUG" (in blue): Functional translation initiation codon (could be other than "AUG"). 5'-CAU-3' (in blue) on the sense RNA: Complement of 5'-AUG-3' on the antisense RNA. Red arrow: Position of the cleavage of the chimeric intermediate. A: Genome-transcribed "dormant" mRNA containing protein-encoding information but lacking in-frame functional translation initiation codon. B: Antisense complement of conventional mRNA. Note that 3'-GUA-5'(in blue) is the 5'-AUG-3'. C: Folding of the antisense RNA strand into self-priming configuration. D: Extension of self-primed antisense strand into sense-oriented sequence followed by strand separation and cleavage (red arrow) of the chimeric intermediate. E: End products of RNA- dependent mRNA amplification. Note that translation of the chimeric RNA end product starts from the antisense RNA- contributed translation initiation codon ("AUG", in blue) and produces a chimeric polypeptide non-contiguously encoded in the genome. 


\section{Activation of Mammalian RNA-Dependent mRNA Amplification: Operational Definition}

The present and several following sections discuss our current understanding of mechanistic and regulatory aspects of the chimeric pathway of mammalian RNA-dependent mRNA amplification. The first stage of this process is its activation. What does the "activation" mean in the context of mammalian mRNA amplification? To formulate its operational definition, it is instructive to review the example of hepatitis delta virus, HDV, an RNA virus that encodes a single protein, hepatitis delta antigen (HDAg). HDAg is neither RdRp nor any other polymerase, yet the virus is capable of vigorous replication in host mammalian cells $[18,19]$. This ability attests to the presence and functionality of RNA-dependent RNA polymerase activity in mammalian cells. Indeed, the RdRp activity was documented in a large variety of mammalian cells and appears to be non-conventional; two possible candidates suggested for this role are the RNA polymerase II complex or its components $[20,21]$ and RdRp activity of the TERT complex [22], both ubiquitously present in all mammalian cells. Under regular circumstances, the RdRp activity in mammalian cells produces only short antisense RNA transcripts. For example, a widespread synthesis of diverse short antisense RNA transcripts initiating at the 3'poly(A) of mRNAs was observed in human cells [23]. It follows that in order to replicate, employing cellular RdRp activity, HDV contributes a factor enabling the production of long transcripts. This factor can only be the HDAg. HDAg was indeed shown to be essential both for the production of long viral transcripts by cellular RdRp, and for the viral replication [24-26]. In its absence only short viral transcripts are generated [24-26]. These observations imply that HDAg constitutes a processivity co-factor of cellular RdRp activity. They also establish a general principle of the requirement for the presence of "inducible" components of the mammalian RdRp complex for its full functionality. Therefore, the activation of mammalian RNA-dependent mRNA amplification can be operationally defined as the assembly of the "competent" RdRp complex capable of transcribing complete complements of the legitimate RNA templates. This, in turn, necessitates either the activation or de novo production of "inducible" RdRp components that are absent or inactive in the cell under regular circumstances. It can be reasoned that, physiologically, the emergence of inducible components of the mammalian RdRp complex is triggered by a variety of cellular stresses and occurs in the context of stress response in general and within the framework of the integrated stress response, ISR, in particular.

\section{Potential Role of the Integrated Stress Response in Activation of the Mammalian mRNA Amplification Process}

The "integrated stress response", ISR, is a complex signaling pathway operating in eukaryotic cells, which is activated in response to a wide range of cellular stresses [27-36]. The "integrating" event in this pathway is the convergence of all stimuli that activate the ISR to the one common point: Phosphorylation of the alpha subunit of eukaryotic translation initiation factor 2, eIF $2 \alpha$, at serine 51 . In mammalian cells, this phosphorylation is catalyzed by members of the family of eIF $2 \alpha$ kinases. This family is comprised of four members: PKR-like ER kinase, PERK; double-stranded RNA-dependent kinase, PKR; general control none-derepressible-2 kinase, GCN2; and heme-regulated kinase, HRI. All four kinases have extensive homology in their catalytic domains but exhibit distinctly different regulatory domains and each requires autophosphorylation and dimerization for full activation [37-42]. As a reflection of their unique regulatory mechanisms, each of these kinases responds to distinct stress stimuli [43]. For example, PERK is located at the endoplasmic reticulum, ER, membrane and is usually activated by ER stress [44-48]. It was also shown to be activated by glucose deprivation, ATP depletion, and oncogene activation [49-52]. GCN2 binds to deacylated tRNAs to become active in response to amino acid deprivation [53] and was also shown to be activated by prolonged glucose deprivation and by UV light [54-56]. PKR is activated mainly by double-stranded RNA, usually during viral infection [57,58]. Other types of stress, such as oxidative and ER stress, growth factor deprivation, cytokines, ribotoxic stress, stress granules, and caspase activity have also been shown to activate PKR in dsRNA-independent manner [59-66]. HRI, unlike other family members that have a broad tissue distribution, is mainly expressed in erythroid cells where it is activated by heme deficiency and is involved in erythroid differentiation [67,68]. It can also be activated, independently of heme, by a variety of other types of stress, including arsenite-induced oxidative stress, heat shock, osmotic stress, proteasome inhibition and nitric oxide [6972]. Recently, HRI activation and, consequently, the occurrence of the ISR were shown to be triggered, via OMA1 to DELE1 to HRI signaling pathway, by mitochondrial distress in a variety of cell types, including neuronal cells $[73,74]$.

Phosphorylation of eIF $2 \alpha$ at serine 51 causes a severe reduction in global cellular protein synthesis, primarily through inhibition of the cap-dependent initiation, while at the same time facilitating cap-independent translation of selected mRNAs, including those encoding specific transcription factors. By the virtue of their ability to interact with nucleic acids, transcription factors are prime candidates for the role of inducible components of the RdRp complex. Alternatively the inducible RdRp co-factors are among products of genes activated by transcription factors produced under the ISR conditions. In turn, the availability of inducible components of the RdRp complex allows the activation of the RNA-dependent mRNA amplification process. This sequence of events is depicted diagrammatically in Figure 7.

The logic of the process illustrated in Figure 7 can be used to explain two documented and one postulated cases of RNAdependent mRNA amplification in mammalian cells. The first one is amplification of mRNAs encoding globin chains in cells undergoing erythroid differentiation $[15,16]$. In this case, it can be envisioned that differentiation-related increase in conventional production of globin polypeptide chains results in sequestration of heme in the hemoglobin tetramer structure, thus forming the hemoglobin. This leads to heme depletion, which triggers the activation of the HRI kinase. Activated HRI kinase phosphorylates eIF2 $\alpha$ at serene 51, thus suppressing total protein production and activating expression of select transcription factors. Either transcription factor(s) themselves or products of some genes activated by them serve as inducible components of the RdRp complex. RNA-dependent amplification of globin mRNAs is activated, yet more globin chains are produced via the mRNA amplification pathway (see relevant discussion at the conclusion of the present section), more heme is sequestered in hemoglobin structures, heme deficiency stress is sustained, the ISR is maintained, and the cycle is perpetuated. 
INTEGRATED STRESS RESPONSE

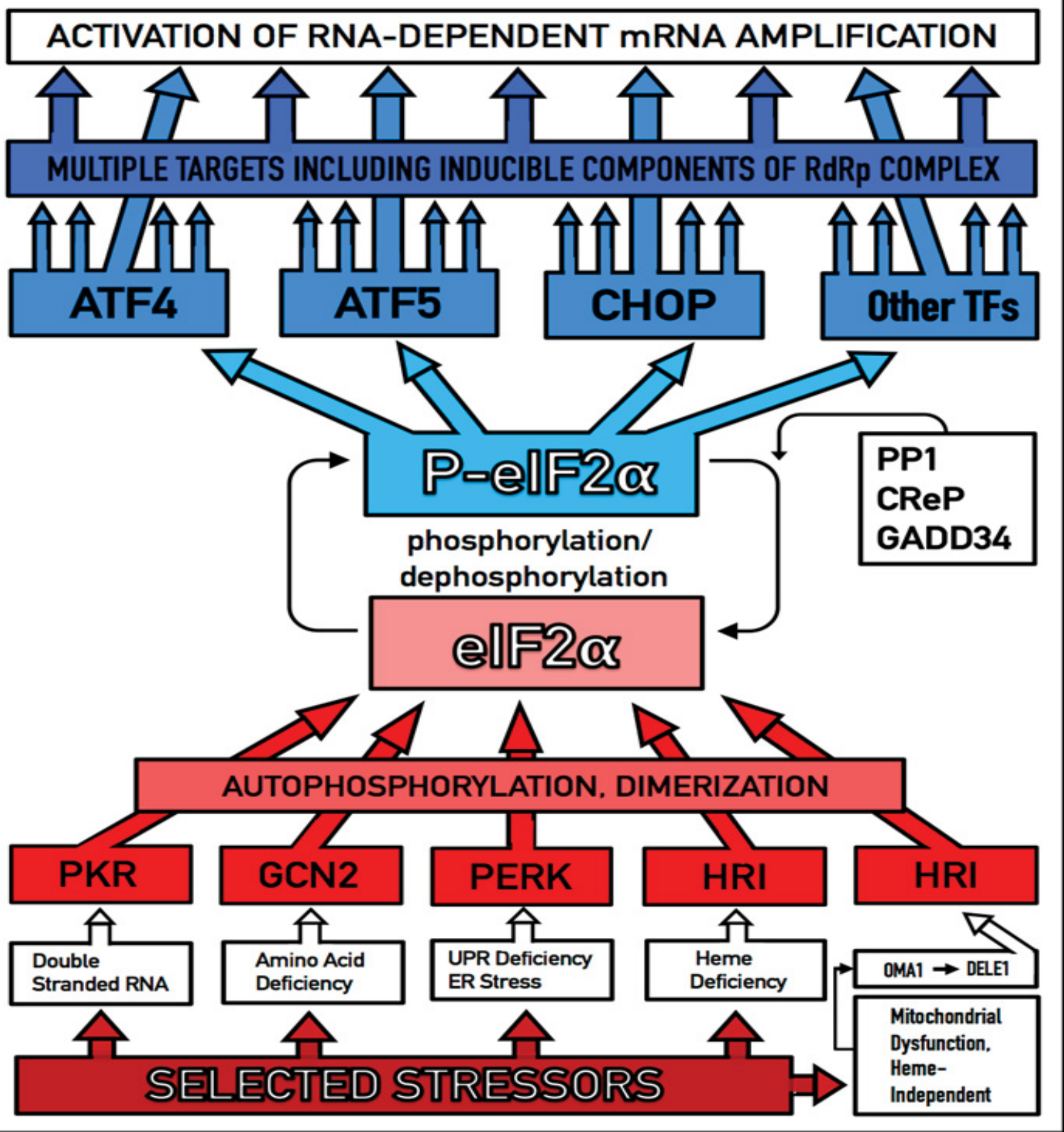

Figure 7: Integrated stress response-mediated activation of mammalian RNA-dependent mRNA amplification. Signals from various cellular stresses, some listed in the Figure, activate, in a stress type-specific manner, one or more members of the family of elF2a kinases: PKR, GCN2, PERK, and HRI. In a number of cases, signaling pathways leading to kinase activation have been elucidated and are described in text. The detailed pathway for heme-independent $\mathrm{HRI}$ activation shown in the Figure depicts mitochondrial stress-induced depolarization activating mitochondrial protease OMA1, which cleaves DELE1 inside of the organelle. One of DELE1 fragments is then released to the cytosol where it binds to and activates HRI. Full activation requires autophosphorylation and dimerization of the kinases. Activated kinases converge on phosphorylation of elF2 $\alpha$ at serene 51 ; this is the "integrating" event and the core of the integrated stress response pathway. elF2 $\alpha$ phosphorylation results in severe global attenuation of cap-dependent translation and also in concomitant initiation of the preferential translation of ISR-specific mRNAs, many encoding transcription factors. Some of these transcription factors, and/or products of genes activated by them, act as inducible components of the RdRp complex; their availability allows assembly of the complex and thus the activation of the mammalian RNAdependent mRNA amplification process. Termination of the ISR is regulated by the constitutively expressed CReP and stress-inducible phosphatase GADD34 that, in conjunction with PP1 phosphatase, dephosphorylate elF2a. 
The second documented example is that of amplification of mRNA encoding alpha1, beta1, and gammal chains of laminin [17] in cells producing and secreting extraordinary quantities of ECM components (in fact, matrigel-producing cells). In this case, physiologically increased conventional expression of genes encoding secreted ECM components "clogs" endoplasmic reticulum and triggers ER stress, which activates PERK. Activated PERK phosphorylates eIF2 $\alpha$, which, in turn, suppresses the bulk of cellular protein synthesis and activates the expression of select transcription factors. Consequently, inducible co-factors of mammalian RdRp are produced, RNA-dependent amplification of laminin-encoding mRNAs is activated, even more laminin polypeptides (and other ECM components) are generated in the mRNA amplification pathway, ER stress is sustained, the ISR is supported, and the whole cycle is self-propagated.

The third example is that of postulated asymmetric amplification of $\beta A P P$ mRNA in Alzheimer's disease [75-82], described in detail below. In this case, a life-long intraneuronal accumulation of conventionally produced beta-amyloid reaches critical levels and triggers mitochondrial dysfunction. This, in turn, activates mitochondrial protease OMA1, which cleaves, still within the mitochondria, protein DELE1. A newly generated fragment of DELE1 is released to the cytosol where it activates the HRI kinase [73,74]. Activated HRI phosphorylates eIF2a, the bulk of cellular protein synthesis is suppressed, select transcription factors are expressed, and the mRNA amplification pathway is activated in neuronal cells. Within this pathway, human beta-amyloid precursor protein-encoding mRNA undergoes asymmetric amplification of Type II described above. It results in a chimeric RNA molecule encoding a C-terminal fragment of $\beta$ APP containing beta-amyloid amino acid sequence at its $\mathrm{N}$-end, the immediate precursor of beta-amyloid peptide, which is generated by gamma-cleavage of the CTF in a non-secretory pathway. As a result, more intraneuronal beta-amyloid is produced via the mRNA amplification pathway, mitochondrial distress persists, the ISR endures, and the cycle is sustained.

Two important notions should be emphasized in the context of the above discussion. First, the ISR could be not the sole activator of mammalian RNA-dependent mRNA amplification. Some types of stress, for example ER stress, activate certain transcription factors by mechanisms distinct from the ISR $[83,84]$. These transcription factors, or products of genes activated by them, could potentially serve as inducible co-factors of mammalian RdRp. The second important notion is that, by the intrinsic logic of the process of ISR-activated mRNA amplification, the cap-less amplified mRNA has to be compatible with phosphorylated eIF $2 \alpha$ and freely translatable under the ISR conditions. Such compatibility could be the consequence of extensive nucleotide modifications of amplified mRNAs and/or their shortened 5'UTRs (on both points see more below). In such a case, amplified mRNA, in addition to being extraordinarily abundant due to the method of its generation, it is also preferentially translated under the ISR conditions, thus augmenting the efficiency of the amplification process.

\section{Generation of Chimeric RNA Intermediates}

Following the activation of the mammalian RNA-dependent mRNA amplification pathway, presumably via the integrated stress response-mediated expression of inducible components of the RdRp complex, the amplification process commences with generation of the complete antisense transcript of a conventionally produced, mature, i.e. spliced, cytoplasmic, amplification-eligible mRNA molecule, as shown in Figure 3, Step 1. The only major prerequisite for an mRNA to serve as the initial amplification template appears to be the presence of the poly(A) segment at its 3 ' terminus $[15,16]$. The vast majority of mammalian mRNA species contains 3'-terminal poly(A) segments. The notion that many, or possibly most, of them could be eligible templates for RdRp was suggested in earlier studies [13]. Subsequent observations by Kapranov and co-investigators showed a widespread synthesis of antisense RNA initiating, apparently indiscriminately, at the 3'-terminal poly(A) of diverse mRNA species in human cells [23]. This, evidently undiscerning, RdRp template eligibility of the bulk of mammalian mRNA species raises questions with regard to mechanisms underlying the manifestly stringent specificity of the mRNA amplification process as seen, for example, in erythropoietic differentiation $[13,15,16]$. The specificity of the amplification process appears to be determined, as discussed below, at the 3' terminus of an antisense RNA transcript by its ability or inability to support production of a complementary sense strand RNA molecule, i.e. amplified mRNA [13,15,16].

The observed, uniformly short, 3'poly(A) region of the chimeric RNA end product of the amplification process $[9,15,16]$, apparently transcribed in a run-off manner from the 5 ' poly(U) segment of the antisense RNA strand, indicates that synthesis of the antisense RNA starts not at the 3' end of mRNA molecules, characterized by a wide size-range of their 3 '-terminal poly(A) segments $[9,15,16]$, but within the $3^{\prime}$ poly(A) segment of the sense RNA strand, in a relatively narrow distance range from the encoded 3'UTR of a conventional mRNA. The manner of antisense RNA initiation remains to be determined; it may involve priming with a uridylated protein, as seen in some viral systems, or occur by a different mechanism. Once transcription is initiated, the RdRp complex proceeds along an mRNA template until a complete antisense RNA complement is produced. Interestingly, at the 3' terminus of the antisense strand, RdRp appears to be capable of transcribing the cap " $G$ " of conventional mRNA despite its inverted orientation [16,17]. The rationale for this conclusion is based on the detected Type One antisense/sense junction sequences of chimeric alpha-globin RNA amplification intermediates $[15,16]$, uniformly containing at the 3 ' termini of their complete antisense portions the " $\mathrm{C}$ " not encoded in the genome, as seen in Figure 1 ; it is summarized in the top panel of Figure 8. If the cap " $G$ " is not transcribed, the antisense strand would terminate with the 3 '-terminal "c" corresponding to the transcription start site of mRNA (highlighted in blue in line A). In such a case, antisense RNA folding/self-priming configuration would be as shown in line A, and, following the extension of self-primed antisense RNA, the antisense/sense junction would consist of the "c/A" (highlighted in blue) as depicted in line B. The experimental results (presented in Figure 1) are different. They show that the sequence of the antisense/sense junction is, in fact, the "cc/A" (line C; highlighted in green). Since the genomic sequence upstream of the transcription start site cannot account for the additional 3 '-terminal " $c$ " in the antisense strand, the remaining possibility is that the " $c$ " in question (line $\mathbf{D}$; highlighted in green) is a transcript of the cap "G" of the sense strand and that the antisense folding into a self-priming configuration occurs as shown in line $\mathbf{D}$.

Similar conclusion was reached in the case of the observed 
amplification of laminin mRNA [17]. It is based on the detected antisense/sense junction sequences of chimeric al laminin RNA amplification intermediates shown in the upper subpanel of the top panel of Figure 2; this conclusion is illustrated in the bottom panel of Figure 8. If the cap "G" is not transcribed, the antisense RNA strand would terminate with the 3'-terminal "u" (highlighted in blue in line $\mathbf{A}$ ) and its folding configuration would be as shown in line A. After the extension, the sense/antisense junction would consist of the " $\mathrm{uC}$ " highlighted in blue and depicted in line $\mathbf{B}$. The experimental results, however, show that the sequence of the sense/ antisense RNA junction is, in fact, the "ucC" highlighted in green in line $\mathbf{C}$. Since the genomic sequence upstream of the TSS cannot account for the additional 3'-terminal " $\mathrm{C}$ " in the antisense strand [85], the only remaining possibility is that the " $\mathrm{C}$ " in question (highlighted in green in line D) is a transcript of the cap " $G$ " of the sense RNA strand and that the antisense RNA folding into a self-priming configuration occurs as shown in line $\mathbf{D}$. It should be emphasized that the above two examples of the capG transcription by RdRp are presented because they are unambiguous. In other cases, like that of beta-globin mRNA amplification, either a "G" occurs in the genome immediately upstream from the TSS, or the antisense component of the sense/antisense RNA junction sequence could be truncated, and the capG-transcribed " $\mathrm{C}$ " removed, in the process of the "chimeric junction shift", a phenomenon discussed below; in these cases, transcription of the capG during generation of the antisense RNA from an mRNA template could be obscured. On the other hand, the two unequivocal examples presented above are convincing enough to warrant generalization.

Following the completion of the antisense RNA complement of a conventional mRNA, strands of the double-stranded RNA structure are separated (Figure 3, Step 2). This is effected by a helicase activity, which mounts the 3 '-terminal poly(A)- containing RNA strand of the double-stranded structure at its 3 ' terminus and proceeds along this strand toward its 5 ' terminus $[15,16]$; strand separation, apparently, can commence while the poly(U)containing antisense RNA strand is still nascent. As the helicase progresses, it, or an associated activity, modifies on average every fifth nucleotide of the poly(A)-containing RNA strand $[15,16]$. Only two species of nucleotides are modified during strand separation; it appears that both are purines, the A and the $G$ $[15,16]$. Moreover, it appears that the same modifying group, with mass number of 37 , is appended to both the $A$ and the $G[15,16]$. The conclusion that both modified bases are purines implies that the modification likely occurs at a position(s) not available in pyrimidine nucleotides. One likely candidate is purine position 3 in the proximity to the Watson-Crick interphase, a position used in the pyrimidines for sugar attachment. The modified chimeric RNA end product appears to be "sticky" in that it forms extensive secondary and tertiary structures [16]. Therefore, the chimeric RNA end product of amplification, containing modified nucleotides, cannot be hybridized to long probes under stringent condition $[15,16]$. Moreover, it appears that the modified nucleotides are either impassable or facilitate the creation of RNA structures that are impassable for a reverse transcriptase. Experimentally, denaturation of the modified chimeric RNA end product of amplification is challenging. The only known method to accomplish this is treatment with methyl mercury hydroxide $[15,16]$. Considering that methyl mercury hydroxide is known to interact with the nucleotide's $\mathrm{NH}$ group during denaturation, and allowing for the mass number requirement of 37, one plausible candidate for the modifying group in question is $\mathrm{LiCH}_{2} \mathrm{NH}_{2}$ $[15,16]$.

At the conclusion of the strand separation stage of the amplification process, a separated conventional mRNA progenitor molecule emerges heavily modified, but otherwise remains intact and can be re-utilized repeatedly as an RdRp template $[15,16]$, whereas its complete antisense RNA complement contains no modified nucleotides [15]. As described below, this outcome, the lack of nucleotide modifications in the antisense RNA transcript, is crucial for the progression of the amplification process.

The generation of the sense RNA strand on the antisense RNA template occurs via the extension of the 3' terminus of a self-primed antisense template and requires the presence within the antisense RNA transcript of two spatially independent complementary elements, whose occurrence in antisense globin RNA molecules was found to be evolutionary conserved across mammalian species [12]. One of these is the strictly 3'-Terminal Complementary Element (TCE), the other is the Internal Complementary Element (ICE). These elements (Figure 3, Step 3) must be complementary to an extent sufficient to form a stable priming structure but may contain both mismatches and unconventional $\mathrm{G} / \mathrm{U}$ pairings. Even if the TCE and the ICE nucleotide sequences are present in the antisense RNA, their proper interaction resulting in a stable self-priming structure is not assured. This is because extensive secondary and tertiary structures, characteristic for any RNA molecule, can obscure and/or interfere with the TCE and the ICE elements and prevent their association. It appears, therefore, that the generation of the sense RNA on the antisense RNA template requires the thermodynamic feasibility, enhanced/enabled by the occurrence of these two complementary and topologically compatible elements, of the antisense RNA strand folding into a stable self-priming configuration. The requirement for terminal localization of the TCE appears to be stringent; an overhang of even a single nucleotide significantly diminishes self-priming efficiency [12]. The crucial importance of the antisense RNA strand remaining unmodified following the separation from its conventional mRNA template (Figure 3, Step 2) plays out at this stage by enabling complementary interactions and the formation of a self-priming structure that otherwise would be impeded. Provided that a self-priming structure is formed, the $3^{\prime}$ end (the TCE/primer element) of the folded antisense strand is extended by the RdRp complex into a sense-orientated RNA molecule terminating with the poly(A) at the 3' end (Figure 3, Step 4), thus generating a hairpin-structured chimeric intermediate consisting of covalently joined sense and antisense RNA strands.

The observed examples of mammalian RNA-dependent mRNA amplification [16,17] described above involve mRNA species transcribed from two types of genes. One type, exemplified by globin chains-encoding genes, contains the "TATA" regulatory element and a rigidly fixed Transcription Start Site (TSS). The other type, exemplified by laminin chains-encoding genes, is TATAless. With the latter, transcription can be initiated from multiple positions and the eligibility for the RdRp-mediated amplification process appears to be regulated, at least in part, by a positional shift of transcription start site. For example, most of the known multiple TSS positions $[86,87]$ in genes encoding all three studied chains of laminin [17] are inconsistent with the eventual generation of 

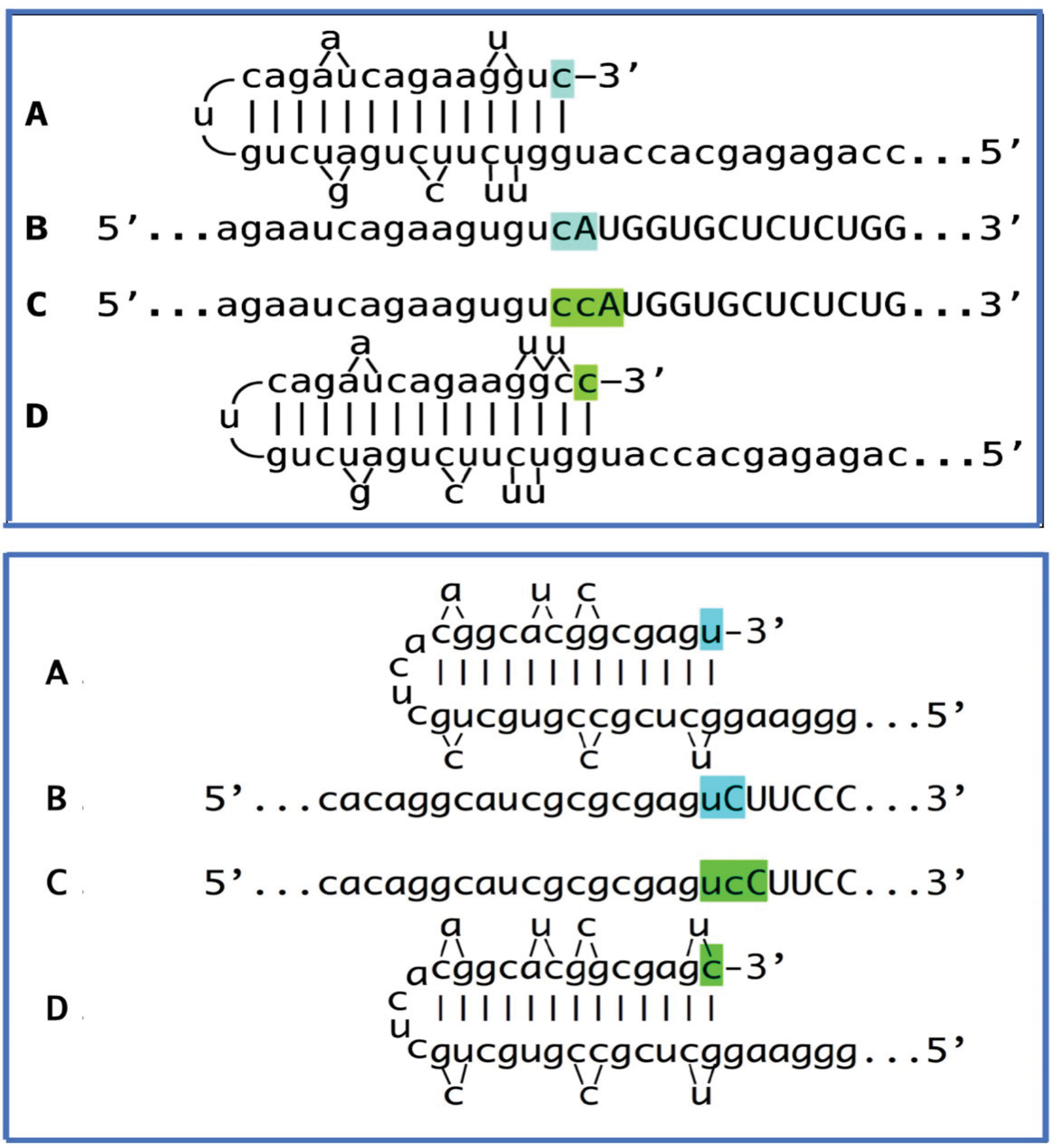

Figure 8: Mammalian RNA-dependent RNA polymerase can transcribe the cap "G" of mRNA. Uppercase letters: Nucleotide sequence of the sense strand; lowercase letters: Nucleotide sequence of the antisense strand. A: Projected self-priming configuration of the antisense RNA strand in the absence of the cap "G" transcription. B: Projected nucleotide sequence of the antisense/sense RNA junction in the absence of the cap "G" transcription. C: Detected nucleotide sequence of the antisense/sense RNA junction. D: Self-priming configuration of the antisense RNA strand as defined by experimental results. Top panel: Transcription of the capG of murine alpha-globin mRNA; data shown is adopted from the top panel of Figure 1. "c" highlighted in blue: 3'-terminal nucleotide (if the capG is not transcribed) of the antisense strand, corresponding to the transcription start site of mRNA; "cA" highlighted in blue: The projected antisense/sense RNA junction structure in the absence of the cap "G" transcription; "c" highlighted in green: Transcript of the cap "G"; "ccA" highlighted in green: The resulting antisense/sense RNA junction structure when the cap "G" is transcribed. Bottom panel: Transcription of the capG of murine alpha1-laminin mRNA; data shown is adopted from the top subpanel of the top panel of Figure 2. "u" highlighted in blue: 3'- terminal nucleotide (if the capG is not transcribed) of the antisense strand, corresponding to the transcription start site ofmRNA; "uC" highlighted in blue: The projected antisense/sense RNA junction structure in the absence of the cap "G" transcription; "c" highlighted in green: Transcript of the cap"G"; "ucC" highlighted in green: The resulting antisense/sense RNA junction structure when the cap "G" is transcribed. Note that in both cases the genomic sequence upstream of the TSS cannot account for the additional 3'-terminal "C" in the antisense strand. 
antisense RNA molecules capable of self-priming within their segments corresponding to the 5'UTRs of mRNA, because positional requirements for the TCE and ICE elements within these molecules are not satisfied. The same is also true for another TATA-less gene, which encodes human beta-amyloid precursor protein and is discussed below. However, a shift in the TSS position may result in compliance with the requirements for the TCE and the ICE and thus can confer on an mRNA the eligibility for the mRNA amplification process. The concepts of such regulation are summarized in Figure 9. If the 3'-distant complementary element of the antisense RNA strand is terminal (Figure 9, panel A), it can form a self-priming structure. If, however, both elements are internal (Figure 9, panel B), the downstream shift of the TSS position can make one of them a 3'-terminal and thus enable selfpriming. When, on the other hand, the antisense RNA strand has no suitable topologically compatible complementary sequences, an upstream shift of the TSS position (Figure 9, panel C) can generate such elements, one of which is 3 '-terminal, and make an mRNA transcript eligible for the amplification process. The events diagrammed in panels $\mathbf{B}$ and $\mathbf{C}$ of Figure 9 can also occur in reverse, making previously eligible mRNAs ineligible for the mRNA amplification process.

\section{Processing of Chimeric RNA Intermediates and Sense/ Antisense "Chimeric Junction Shift"}

The hairpin-structured chimeric intermediate of mRNA amplification consists of covalently joined sense and antisense RNA strands and, therefore, contains an extensive double-stranded region (Figure 3, Step 4). The double-stranded portion of such a structure is separated by a helicase activity invoked above, which mounts the 3'-terminal poly (A) of a newly synthesized sense strand RNA component of the chimeric intermediate and proceeds along this RNA strand in the $5^{\prime}$ direction modifying the molecule as it advances (Figure 3, Step 5). When the helicase complex reaches a single-stranded portion of the hairpin structure, it, or an associated activity, cleaves the molecule either within the TCE, at a TCE/ICE mismatch or, if there are no mismatches, immediately upstream of the TCE; the cleavage was shown to occur between the 5' hydroxyl group and the 3 ' phosphate $[15,16]$ (red arrow, Figure 3, Step 6). The cleavage releases the chimeric RNA end product with shortened 5'UTR and the 3'-truncated antisense RNA (Figure 3, Step 7). If the cleavage were to occur at a TCE/ICE mismatch close to and upstream of the $3^{\prime}$ end of the TCE, i.e. close to the $3^{\prime}$ end of the antisense RNA component of the chimeric intermediate, and a substantial portion of the TCE was retained in the antisense RNA, the self-priming configuration of the antisense RNA could remain stable and be re-used, apparently after 3'-P to 3'-OH phosphatasemediated conversion, to generate another chimeric intermediate. In such a case, the site of the antisense/sense RNA junction would shift upstream from the previous one by the size of the antisense RNA truncation resulting from the initial cleavage, a phenomenon we refer to as the "Chimeric Junction Shift" If such a process occurs more than once, multiple antisense RNA truncations are generated, and every time the position of the antisense/sense RNA junction is shifted upstream. In this context, multiple versions of chimeric intermediate, differing by the sizes of the 3' truncation of their antisense RNA components, would co-exist concurrently. Eventually, the substantial portion of or the entire TCE element would be cleaved off the antisense RNA, and it could not be reutilized to generate a chimeric intermediate. In fact, such a process of sequential cleavages at different TCE positions was observed with chimeric intermediates of globin mRNAs amplification. As can be seen in Figure 1, one shift of the antisense/sense junction was observed with alpha-globin RNA chimeric intermediates and two shifts were seen with beta-globin RNA chimeric intermediates, thus explaining the origin of the observed chimeric junction sequences with 3'-truncated antisense RNA components. The observed "Chimeric Junction Shift" phenomenon also explains the evident absence of the capG-transcribed 3'-terminal " $\mathrm{C}$ " in the antisense components of some chimeric intermediates of the mRNA amplification process.

The Chimeric RNA End Product of Mammalian RNA Dependent mRNA Amplification Is a Proverbial "Dark Matter" mRNA, Whereas Chimeric RNA Intermediates Are Readily Detectable by Conventional Methods of Analysis

If the "visualization" of a nucleic acid species is defined as its detection by hybridization to a labeled complementary probe, or the determination of its nucleotide sequence, the modified chimeric RNA end products of the chimeric mRNA amplification pathway can be categorized as a proverbial "Dark Matter" mRNA because it is invisible to the above methods of detection, i.e. hybridization under stringent conditions or nucleotide sequencing based on the reverse transcription of RNA of interest. This is because, as was mentioned above, nucleotide modifications of the chimeric RNA end product of amplification interfere with and prevent both, hybridization under stringent conditions and reverse transcription-based nucleotide sequencing. In this context, therefore, it is important to lucidly define which constituents of the chimeric mRNA amplification pathway containing its distinguishing feature, the "identifier", i.e. sense/antisense RNA junction sequence, can and which cannot be detected by reversetranscription-based nucleotide sequencing. In the chimeric pathway of mRNA amplification, the cleavage of the chimeric intermediate following strand separation and the associated modifications of its poly(A)-containing chimeric component (sense-oriented RNA and either a portion of or the entire TCE element of the antisense RNA) is the ultimate act in the generation of the chimeric RNA end product. Consequently, it is formed and released already modified $[15,16]$ and, unlike its hairpinstructured chimeric intermediate precursor, is never present in the unmodified or even partially unmodified form and, therefore, cannot be detected by sequencing methods dependent on the lack of modifications. On the other hand, once the extension of the 3' terminus of unmodified self-primed antisense RNA strand commenced, the antisense/sense RNA junction, the "identifier", is generated and it remains unmodified, and therefore detectable, until the extension process is completed. Moreover, only when the extension of the self-primed antisense RNA strand into the senseorientation RNA molecule is concluded by the synthesis of the 3 ' poly (A), transcribed from the $5^{\prime}$ poly $(\mathrm{U})$ of the antisense strand, thus forming a double-stranded poly(A)/poly(U) structure, can the strand separation and accompanying nucleotide modification begin. Accordingly, following the completion of the extension and the commencement of strand separation, the sense/antisense RNA chimeric junction remains further not modified for the duration of the strand separation process downstream from the junction region. Thus, once the junction is created, the time required for the completion of the extension process, cumulatively with that 


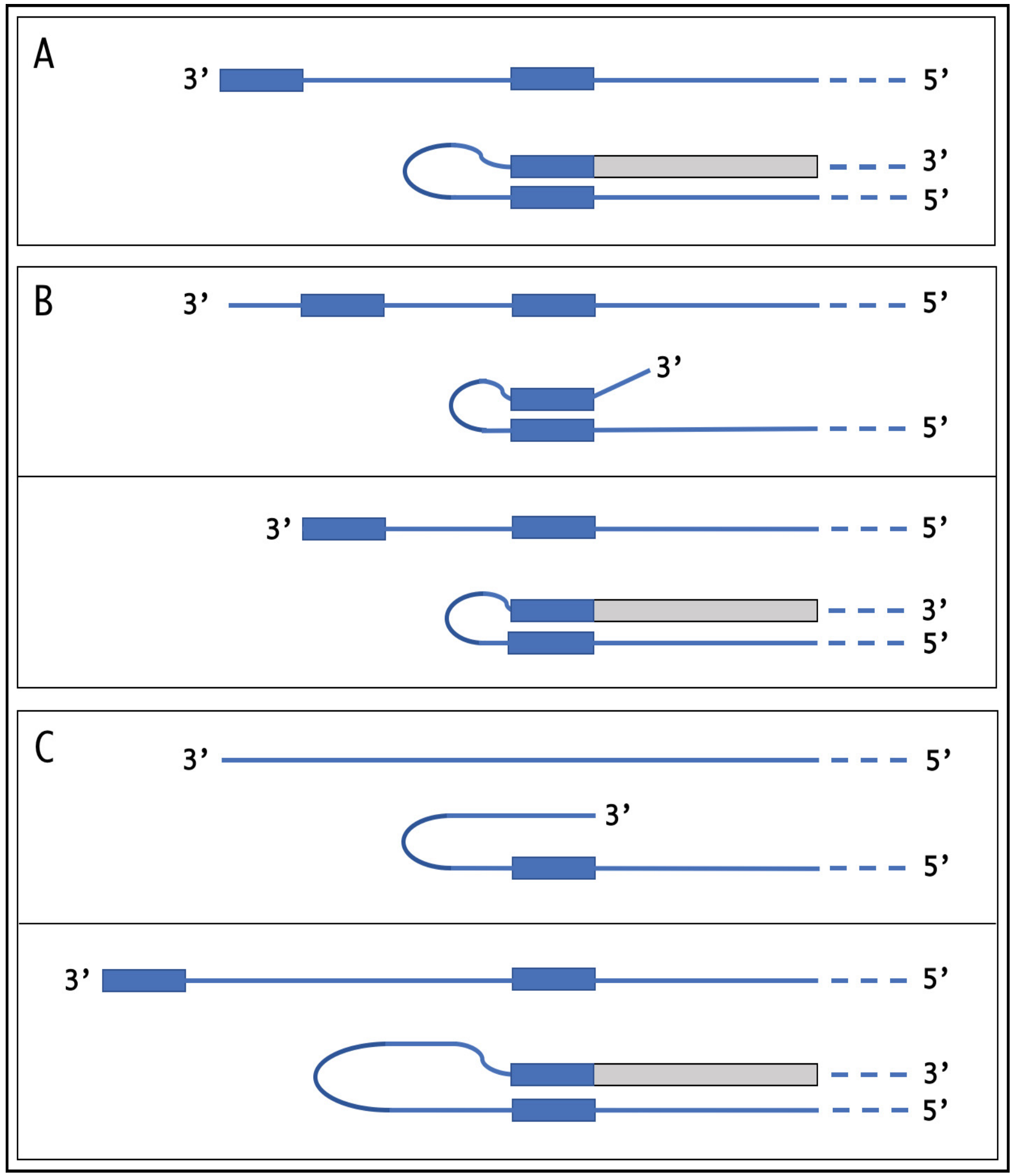

Figure 9: TSS shift as potential regulator of the eligibility of an mRNA for the amplification process. Single line: 3 'terminus of the antisense RNA strand. Filled grey boxes: Sense RNA strand. Filled blue boxes: Topologically compatible complementary elements on the antisense RNA strand. A: One of the complementary elements is 3'-terminal; folding results in a self-priming structure that is extended into the sense strand. B: Both complementary elements are internal, no self-priming is possible (top subpanel); TSS shift in the downstream direction makes one of the elements 3'-terminal and allows self-priming of the antisense RNA and its extension into the sense strand (bottom subpanel). C: There are no complementary elements, no self-priming structure is formed, no extension occurs (top subpanel); TSS shift in the upstream direction generates 3'-terminal complementary element and thus enables self-priming and extension (bottom subpane/). Note that processes depicted in panels $\mathbf{B}$ and $\mathbf{C}$ can occur in reverse, resulting in a loss, rather than the acquisition, of the eligibility. 
required for the strand separation and associated nucleotide modifications downstream from the junction region, constitute a temporal window of opportunity enabling the occurrence of the yet unmodified sense/antisense RNA junction sequences at a certain steady-state level and their detection by conventional hybridization/reverse transcription-based methods.

\section{Possible Functions of Nucleotide Modifications in the Chimeric RNA End Product of Tier One}

The functions of nucleotide modifications occurring during mRNA amplification remain to be elucidated. They could include the facilitation of double-stranded RNA strand separation, stimulation of the cap-independent translation of the cap-less chimeric mRNA end product of amplification, and regulation of its stability. The latter is suggested by a drastic decrease in levels of the chimeric RNA end product of globin mRNA amplification, but not of conventionally produced globin mRNA, during the transition from the erythroblast stage, characterized by rapid hemoglobin accumulation, to the reticulocyte stage where the levels of hemoglobin are only maintained [16] and conventionally produced globin mRNA is apparently sufficient. Arguably, the most important function of nucleotide modifications within the chimeric RNA end product of the amplification process could be that they render the modified mRNA compatible, unlike the bulk of conventional cellular mRNA, with phosphorylated eIF2 $\alpha$. This implies that in addition to being extraordinarily abundant due to the method of its generation, the amplified, and thus modified, mRNA is also preferentially translated under the ISR conditions, thus augmenting the efficiency of the amplification process. The compatibility with phosphorylated eIF $2 \alpha$ also implies a different mode of translation than that of conventional mRNA. Indeed, the modified RNA end product of the amplification process showed strong affinity to ribosomal RNA $[15,16]$. It appears that it forms complexes with ribosomal RNA where modifying groups are sequestered, enabling a codon/anticodon interaction [9]. Shortened 5'UTRs, characteristic for the chimeric RNA end product of amplification [15,16], could also play a role in preferential translation of amplified mRNA under the ISR conditions.

\section{Principal Players of the Chimeric mRNA Amplification Pathway and Their Functions}

Beside the competent RdRp complex that includes its inducible components, generates complete complements of conventional mRNA, and is capable of extending self-primed antisense RNA, the principal players in the chimeric pathway of mammalian RNAdependent mRNA amplification are: The initial amplification template, a conventional gene-transcribed, amplification-eligible mRNA progenitor molecule, and two distinct RNA end products of the amplification process, namely the chimeric RNA end product and the antisense RNA end product, a 3'-truncated antisense RNA complement of the initial mRNA amplification template. Of these three types of RNA molecules, only the first, a conventional mRNA progenitor, can be used repeatedly in the amplification process. This is because only this molecule can be recurrently transcribed, acquiring nucleotide modifications when utilized as a template and separated from its transcript the first time but otherwise retaining its complete integrity, into the antisense RNA containing both elements, the TCE and the ICE, required for the progression of the amplification process. The resulting chimeric
RNA is the "functional" end product, the amplified mRNA, i.e. in most cases it can be translated into a polypeptide. It cannot, however be amplified further in the chimeric pathway because its possible antisense transcript would lack the TCE element and would be, therefore, incapable of self-priming/extension. This leaves the 3'-truncated antisense RNA end product. It also cannot be amplified further in the chimeric pathway because it lacks either a substantial portion of or the entire TCE element (transferred to the chimeric RNA end product; the source of the chimerism). Moreover, it apparently has neither role nor function in Tier One of the mRNA amplification process. Is it, then, an inconsequential "by-product" of the chimeric mRNA amplification pathway? The answer, communicated below, is resoundingly negative.

\section{Mammalian RNA-Dependent mRNA Amplification: Tier Two, Physiologically Occuring Intracellular Polymerase Chain Reaction, IPCR \\ Detection of Uniformly 5'-Truncated and Polyurydilated Globin mRNA Sequences}

In the study of RNA-dependent amplification of globin chains-encoding mRNAs [16], in addition to testing for the presence of chimeric RNA junctions containing both sense and antisense components, a second general search was conducted for 5 '-truncated, sense-orientated globin mRNA sequences containing poly $(\mathrm{U})$ segments appended to their truncated 5' termini. The primary reason for this search was the observation by Kapranov and co-investigators [23] of a class of such mRNA molecules in human cancer cells where a robust synthesis of antisense RNA, initiating at the 3'-terminal poly(A) segments of their sense-oriented mRNA counterparts, was also detected. It was reasoned $[15,16]$ that a connection might exist between these two observations and, as discussed below, it appears that it indeed does. In the search for 5 'poly(U)-containing globin mRNA molecules [16], cytoplasmic RNA from murine differentiating erythroid tissues was analyzed by directional next generation RNA sequencing. A selection of 5'-terminal poly $(\mathrm{U})$-containing fragments detected for both alpha- and beta-globin mRNA is presented in Figure 10 (lines G, $\mathbf{H}$, and $\mathbf{I}$ highlighted in gray in both top, alpha-globin, and bottom, beta-globin, panels) together with projected chimeric pathways for alpha- and beta-globin mRNA amplification. In all detected sequences, globin genome-encoded portions are 5'-truncated. The truncations are not random. Interestingly, they are rather uniformly clustered around the nucleotide positions corresponding to the projected predominant truncations of the antisense RNA "byproduct" of the chimeric amplification pathways for both alphaand beta-globin mRNA. The implications and significance of these observations, and their relevance to the processes of mammalian RNA-dependent mRNA amplification are addressed below.

\section{The iPCR Pathway of Mammalian mRNA Amplification: Physiologically Occurring Intracellular Polymerase Chain Reaction}

What could be the origin of sense-oriented mRNA sequences with non-conventionally templated 5'-terminal poly(U) appended to the 5'UTRs of mRNA truncated at positions corresponding to the apparent cleavage sites within the antisense components of the chimeric intermediates? As discussed above, the accumulated data strongly suggest that the only major requirement for an RNA molecule to serve as mammalian RdRp template is the presence of 


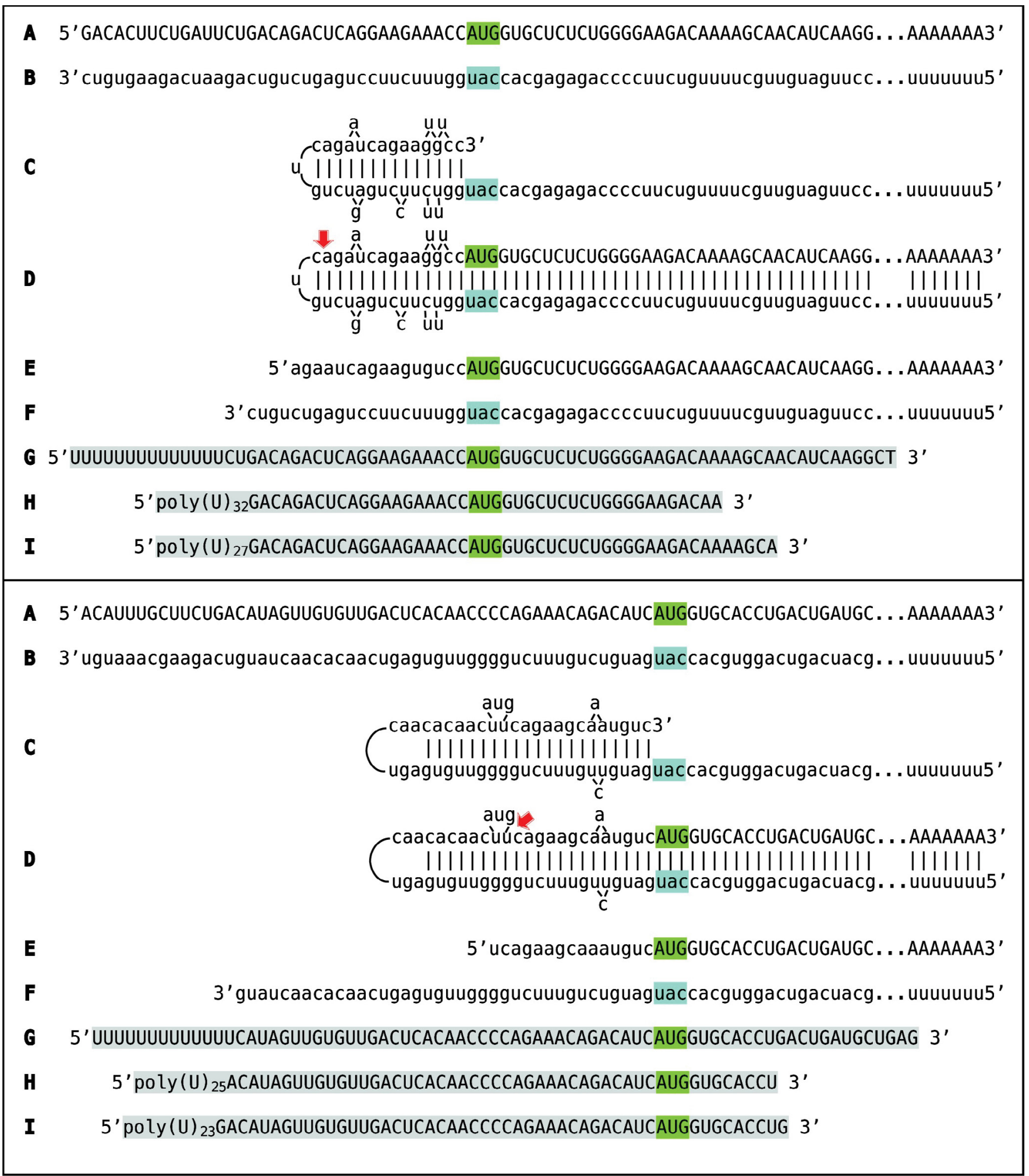

Figure 10: Detection of 5'-truncated sense strand murine globin mRNA sequences containing 5'-terminal poly(U) segments. Uppercase letters: Nucleotide sequence of the sense RNA strand; lowercase letters: Nucleotide sequence of the antisense RNA strand. Highlighted in green: "AUG" translation initiation codon on the sense RNA strand; highlighted in blue: "uac" complement of translation initiation codon on the antisense RNA strand. Red arrows: positions of cleavage of the chimeric intermediate. A-F: Projected pathways of the chimeric Tier of globin mRNA amplification. A: Conventional genomeoriginated mRNA; B: Antisense complement of conventional mRNA; C: Folding of the antisense RNA strand into self-priming configuration, the 3'terminal "c" is a transcript of the 5'capG of mRNA; D: Extension of self-primed antisense RNA strand into sense-oriented sequence. E: Projected chimeric RNA end product. F: Projected cleavage-generated 3'-truncated antisense RNA.G, $\boldsymbol{H}$, and $\boldsymbol{I}$ : selection of detected 5'-truncated sense strand RNA sequences containing 5'-terminal poly $(\mathrm{U})$ segments (highlighted in grey). Note that truncations are clustered around the nucleotide positions corresponding to the projected truncations of the antisense RNA by-product of the chimeric amplification pathways. Top panel: Alpha-globin RNA sequences; bottom panel: Beta-globin RNA sequences. 
the poly(A) segment at its 3' terminus. Provided the presence of the 3'-terminal poly(A) in an RNA molecule is necessary and sufficient for initiation of RNA-dependent RNA synthesis in mammalian cells, in addition to the chimeric pathway of mammalian RNAdependent mRNA amplification, another mRNA amplification paradigm, constituting Tier Two of the amplification process, may be considered. If an antisense transcript is polyadenylated at the 3 ' terminus by a known or a novel cellular poly(A) polymerase, it would become a valid template for mammalian RdRp. Since the antisense RNA strand has, by virtue of its initiation within the 3' poly $(\mathrm{A})$ region of a conventional mRNA, the poly $(\mathrm{U})$ stretch at the 5 ' terminus, its transcription by the RdRp complex would result in a sense strand with the $\operatorname{poly}(\mathrm{U})$ at the 5 ' end and the poly(A) at the 3 ' end, also a legitimate mammalian RdRp template. Since strand separation mechanisms (helicase complex and associated activities discussed above) are in place and the described sequence of events can occur repeatedly, the process will amount to an intracellular polymerase chain reaction, iPCR. If, in such a process, the initial 3' polyadenylation of the antisense RNA is coupled and occurs in conjunction with, and at the site of, the cleavage of the chimeric intermediate, the 5' truncation of the gene-encoded portion of the sense-oriented mRNA transcript from such an antisense RNA template would correspond precisely to the 3' truncation of the antisense RNA end product ("by-product") of Tier One of mRNA amplification, exactly so as was observed experimentally (Figure $10)$.

Such sequence of events is presented diagrammatically in Figure 11. Up to the Step 6, all processes occur exactly as are shown in Figure 3 and described above. In steps 6/7, the cleavage at a TCE/ICE mismatch or at the 5 ' end of the TCE (red arrow) is coupled with polyadenylation of a newly generated 3' terminus of the truncated antisense RNA strand. This event marks a conclusion of the chimeric cycle of amplification. One of its end products, the chimeric RNA marked in Figure 11 as "END PRODUCT, TIER ONE", is identical to the chimeric end product of Figure 3. The other end product of the chimeric pathway of mRNA amplification is different from its equivalent in Figure 3. It is the antisense RNA strand, 3'-truncated in the same position as depicted in Figure 3, but in addition to the 5 '-terminal poly(U), it also contains the 3 '-terminal poly(A) segment (Figure 11, Step 7). This molecule constitutes the initial template of the intracellular polymerase chain reaction, its "progenitor". Indeed, just as in Step 1, RdRp activity initiates transcription within the 3'poly(A) of the antisense RNA template and generates the sense RNA strand containing both the 5'poly(U) segment and the 3'poly(A) segment (Figure 11, Step 8). After strand separation (shown in Figure 11, Step 9 in a simplified form, since strand separation probably commences while the poly(U)-containing RNA strand is still nascent), there are now two templates, each containing the 3 '-terminal poly(A) as well as the 5 'terminal poly(U) segments (Figure 11, Step 10; note that only one of possibly multiple iPCR round is shown), and the iPCR is under way. Strand separation and associated nucleotide modifications probably commence at the 3'-terminal poly(A) as soon as it becomes double-stranded, i.e. when the poly(U)-containing RNA strand is still nascent or even when only a sufficiently long 5 '-terminal poly(U) segment has been generated; in such a case, the helicase complex would be trailing closely the progression of the RdRp complex. Therefore, as detailed further in the "Mechanistic Aspects" section below, at steady-state there would be many more not yet modified, and thus detectable, 5 '-truncated, -terminal poly(U)-containing sense RNA strand sequences than 3'-truncated, -terminal poly(A)-containing antisense RNA strand sequences; both types of sequences are uniquely specific for the iPCR pathway and constitute its "identifiers". The above considerations apply equally to the opposite termini of the same iPCR-amplified molecules: At steadystate there would be many more unmodified 5 '-terminal poly(U)containing antisense RNA sequences than 3 '-terminal poly(A)containing sense RNA sequences; these types of sequences, however, would be indistinguishable from corresponding segments of either conventional mRNA or intermediates and products of the chimeric pathway.

\section{Mechanistic and Regulatory Aspects of Tier Two of Mammalian RNA-Dependent mRNA Amplification, the iPCR Pathway}

Conceptually, amplification of a nucleic acid molecule by a polymerase chain reaction necessitates, beside the presence of the building blocks, the occurrence of a template, the priming arrangement for the initial nucleic acid strand and for its complement, a suitable polymerase, and the procedure for strand separation that doubles the number of template molecules in each cycle. In a conventional PCR reaction, a single-stranded DNA molecule serves as a template, oligonucleotides complementary to the initial DNA strand and to its complement in desired and appropriate positions function as primers (the "forward" and the "return" primers), DNA polymerase of choice extends the 3' end of a primer, generating a double-stranded molecule, and a thermal treatment separates the strands to enable the next cycle of the chain reaction where both complementary DNA strands serve as templates; with each cycle the number of molecules, and thus of templates, in the reaction doubles.

In the intracellular PCR process, iPCR, the eligible initial template is a single-stranded RNA molecule, the 3'-truncated antisense RNA end product of the chimeric mRNA amplification pathway, containing the poly(A) segment at its 3 ' terminus and the $\operatorname{poly}(\mathrm{U})$ segment at its 5 'terminus. The process is driven by the mammalian RdRp complex, which initiates transcription within the poly(A) segment and generates a complete complement of the initial amplification template. The priming arrangements for both the initial template and its complement are reflected in the template's RdRp eligibility requirements that are satisfied by the occurrence of the poly(A) segment at the 3 ' termini of both the initial RNA template as well as of its transcript/complement, and strand separation is carried out by the helicase/modifying activity described above in the discussion of Tier One of mRNA amplification. In the chimeric pathway, when a full-length 5' poly $(\mathrm{U})$-containing antisense RNA strand is generated (transcribed by the RdRp complex from a conventional mRNA progenitor) and separated from its template (steps $\mathbf{1}$ and 2, Tier One, Figure $11)$, it is not an iPCR-eligible template because it lacks the 3'-terminal poly(A) segment. Instead, provided that it contains the topologically compatible 3 '-terminal and internal complementary elements, it self-primes its extension into a sense RNA strand; thus generating the intermediate in the chimeric pathway of mRNA amplification. It is the processing of this intermediate that has the potential to produce, in addition to the chimeric RNA end product, the iPCR-eligible template. This requires chimeric intermediate 


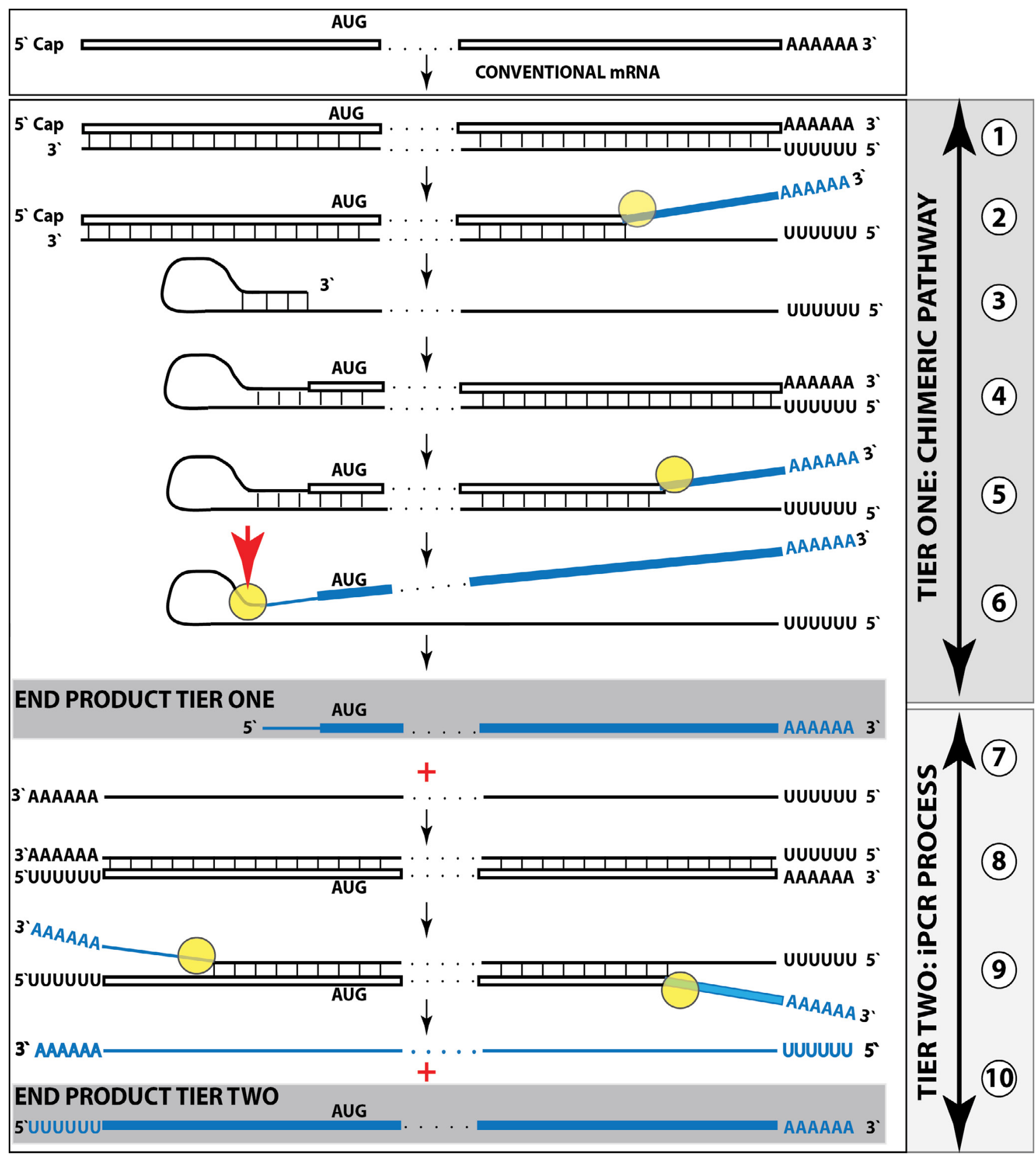

Figure 11: Mammalian RNA-dependent mRNA amplification is a Two-Tier process. Top panel: Conventional, genome- originated mRNA molecule. Bottom panel: Tier One, the chimeric pathway, progressing into Tier Two, the iPCR pathway. Boxed line: Sense strand RNA. Single line: Antisense strand RNA. "AUG": Functional translation initiation codon (could be other than "AUG"). Yellow circle: Helicase/modifying activity complex. Blue lines (both single and boxed): RNA strands, both sense and antisense, modified and separated from their complements by a helicase complex. Red arrowhead: Position of cleavage of the chimeric intermediate. Step 1: Synthesis of antisense RNA strand; step 2: Strand separation; step 3: Folding of the antisense RNA strand into self-priming configuration; step 4: Extension of self-primed antisense RNA into sense RNA; step 5: Strand separation; step 6: Cleavage of the chimeric intermediate coupled with 3'polyadenylation of the truncated antisense RNA; step 7: End-products of the chimeric pathway of the mRNA amplification process; step 8: RdRpmediated synthesis of the sense strand initiated at the 3'poly(A) of antisense RNA; step 9: Strand separation; step 10: iPCR products. Each RNA strand constitutes an iPCR template. The antisense RNA can be further amplified whereas the sense RNA strand can be used either for amplification or for translation. Note that the Figure is simplified: (a) Initiation of transcription at the 3'-terminal poly(A) apparently occurs within the poly $(A)$ segment rather than at its end; (b) Strand separation shown in steps $\mathbf{2}$ and $\mathbf{9}$ apparently commences while the poly(U)-containing RNA strand is still nascent, as described in text. 
cleavage-coupled 3' polyadenylation of the cleavage-truncated antisense RNA strand, which already contains the 5'-terminal poly(U) segment transcribed from the 3'-terminal poly(A) of a conventional mRNA progenitor molecule. The presence of the 3'-terminal poly (A) segment on the antisense RNA would allow the RdRp complex to initiate its transcription and to proceed with the synthesis of the sense RNA strand complement that commences with the 5'-terminal poly(U) and concludes with the 3'-terminal poly(A) segments, also an iPCR-eligible template. The following separation of strands by the helicase/modifying activity would double the number of templates and enable the next cycle of a polymerase chain reaction. Thus, the key feature underlying the feasibility of iPCR is that both the initial cleavage/polyadenylationreleased antisense RNA molecule and its transcript are eligible RdRp templates. Potentially and purely hypothetically, if an antisense RNA transcribed from a conventional mRNA were polyadenylated at the 3 ' end upon conclusion of its transcription, it would, after strand separation, become an eligible iPCR template. This, however, is highly unlikely physiologically, because such a process would completely lack the specificity. In the Two-Tier amplification process, because the generation of the initial iPCR progenitor template is coupled with and enabled by the concluding step of the previous Tier, the specificity of the iPCR pathway is precisely equal in its stringency to that of the preceding chimeric pathway, which, in turn, is defined by the occurrence within an antisense RNA transcribed from a conventional mRNA of the topologically compatible TCE and the ICE features and by the thermodynamic feasibility allowing the initial antisense transcript to properly fold and self-prime its extension. On the other hand, importantly, whether or not Tier Two of mammalian mRNA amplification, iPCR, occurs does not affect in any way Tier One, the chimeric pathway.

The translationally functional end product of the iPCR amplification pathway is a non-chimeric sense strand RNA identical to a conventional genome-encoded mRNA in informational content and in all other attributes except three - it contains modified nucleotides, it is 5'-polyuridylated (and apparently not capped), and it is $5^{\prime}$-truncated in its genome-encoded portion. The truncations are no larger than the TCE of a corresponding antisense RNA, and since TCEs appear to be relatively short, significantly shorter than a typical mammalian 5'UTR, so would the 5' truncations be. Mechanisms underlying the iPCR Tier of amplification could explain the not yet elucidated observations in human cancer cells of a class of unconventional mRNA sequences that differ from their conventional counterparts only in two aspects: (a) their genome-encoded portions are truncated at the 5' end, typically by 14 to 18 nucleotides, not affecting their protein-encoding capacity, and (b) they contain poly(U) segments appended to their truncated 5' termini [23]. In terms of the Two-Tier mRNA amplification process, such molecules could be products of the second Tier of amplification, the iPCR pathway, and the extent of 5' truncations in their genome-encoded portions could reflect the average size of the antisense 3' truncation at the conclusion of the preceding chimeric pathway Tier, i.e. the distance between the cleavage site within the chimeric intermediate and the 3 ' end of the antisense strand, or, in other words, the average size of their TCE elements.

Whereas the occurrence and regulation of Tier One of mammalian mRNA amplification, the chimeric pathway, is entirely independent from Tier Two, the iPCR process, the occurrence of the latter depends on, and is impossible without, the completion of the former. Moreover, the occurrence of the iPCR also requires that the last step of the chimeric pathway, the cleavage of the chimeric intermediate, were coupled with the 3'polyadenylation of the cleavage-truncated antisense RNA strand. Arguably, the efficiency of the iPCR pathway may vary in a regulated manner in accordance with the needs for the production of a protein encoded by an mRNA species being amplified. Considering that the presence of the 3 '-terminal poly(A) segment is presumably a single major template eligibility requirement for the initiation of RdRp-mediated RNA-dependent RNA synthesis [13,15,16], and allowing for the operational presence of the RdRp complex, a key enzymatic component of both the chimeric pathway and the iPCR process, it is reasonable to suggest that the principal regulatory checkpoint for the entry into Tier Two of mRNA amplification could be the rate of the chimeric intermediate cleavage-coupled 3' polyadenylation of the cleavage-truncated antisense RNA strand. In the extreme case, if the polyadenylation (but not the cleavage) were completely suppressed, so would Tier Two be, but neither the outcome nor the efficiency of the Tier One process would be affected in any way.

In the chimeric pathway of mammalian mRNA amplification, the RdRp complex appears to initiate transcription within the 3'-terminal poly(A) segment rather than at its 3' end. Possibly, the initiation "within" the poly(A) segment is not a stringent requirement, and only the distance from the encoded 3'UTR of mRNA is important; then RdRp can also initiate at the end of the 3'poly(A) of the right size range. Otherwise, it can be assumed that the initiation within the 3 'poly $(\mathrm{A})$ is true for the iPCR process as well as for the chimeric pathway. In such a case, successive iPCR rounds would result in the shortening of both the 5'-terminal poly(U) segments and, consequently, the 3'-terminal poly(A) segments. This, in turn, would eventually lead to the loss of template's RdRp eligibility unless the 3'-terminal poly(A) elongation, known to occur in the cell, takes place in parallel with its shortening. This is in contrast to the chimeric mRNA amplification pathway where repeated utilization of conventional mRNA as a template for the antisense RNA synthesis apparently has no impact on the length of its 3'-terminal poly(A). Additional augmentation of the efficiency of the iPCR pathway could occur at the level of the iPCR templates availability, which is comprised of two components: stability of both sense and antisense strands produced in the reaction and the utilization of the mRNA end product (sense-oriented strand) of the second Tier. The iPCR is, as any chain reaction, potentially an exponential process provided that the entire output of every cycle is utilized as templates in the next cycle of a chain reaction. However, if all sense strand RNA products in every cycle were withdrawn due to the recruitment for protein synthesis, this Tier of amplification would be linear, akin to the chimeric pathway. In all probability, some sense-oriented iPCR-produced molecules are removed for translation and some are utilized as templates for further amplification, making the process faster than linear but slower than exponential. As for the stability of the iPCR templates, it could be regulated, at least in part, by nucleotide modifications, present in both RNA strands, as was discussed in the "chimeric pathway" sections above.

Considering that the RNA-dependent mRNA amplification process proceeds trough the double-stranded RNA stages, 
an obvious question is how it evades (if it does) the dsRNA response? One possibility is that the amplification process is compartmentalized and the components of the dsRNA response have no access to it. Another possibility is the involvement of factors protecting from the dsRNA response. What is known experimentally, is that unmodified double-stranded RNA components associated with RNA-dependent mRNA amplification appear to be extremely short-lived as reflected in their very low steady-state levels $[8,9]$. This means that strand separation and associated nucleotide modifications occur very rapidly and double-stranded molecules may have very limited exposure. Moreover, provided that one of functions of nucleotide modifications is strand separation, when already modified RNA is used as a template for RdRp, as in the re-use of conventional mRNA in Tier One, or as occurs routinely in Tier Two, the iPCR pathway, strand separation would take place concurrently with the progression of the RdRp complex and no double-stranded RNA exposure would result. An interesting alternative possibility is that the occurrence of both Tiers of mammalian RNA-dependent mRNA amplification generates a certain steady-state level of double-stranded RNA, which, in turn, supports a steady-state population of activated double-stranded RNA-dependent kinase PKR, a member of the family of eIF2a kinases. Consequently, eIF $2 \alpha$ is phosphorylated, the integrated stress response elicited, the production of inducible components of the RdRp complex sustained, and the mRNA amplification process perpetuated.

Whereas in Tier One only the chimeric RNA end product is modified because the hairpin-structured chimeric intermediate contains a single linear double-stranded 3'poly(A)/5'poly(U) region, a requirement and the starting point for the commencement of the strand separation/modification (initiating on and proceeding along and modifying the poly(A)-containing strand), in the iPCR pathway such regions are present at both ends of a linear double-stranded structure. Accordingly, strand separation and associated nucleotide modifications commence on the poly(A) segments of both, sense and antisense, RNA strands and, therefore, both strands are eventually modified. The distinguishing features of the iPCR pathway Tier, its "identifiers", are the 3 '-terminal region of the cleavage-truncated (or transcribed from the sense 5'poly(U)/3'poly(A)-containing RNA in Tier Two) antisense strand RNA containing the 3 '-terminal poly(A) segment, and the 5 '-terminal region of its corresponding sense strand RNA transcript, with a 5' truncation mirroring a 3 ' truncation of the antisense strand and containing the 5 '-terminal poly(U) segment; both "identifiers" are, in fact, another kind of "junctions". Unlike the chimeric pathway, where the chimeric RNA end product is never present even in partially unmodified form, and its "identifier", a sense/antisense RNA junction, cannot be detected by reverse-transcription based methods, the iPCR pathway presents possibilities to detect both unmodified "identifiers" of Tier Two in both of its RNA end products. The detection opportunities, however, are dissimilar for the sense and the antisense RNAderived iPCR “identifiers". The 3'-truncated and polyadenylated antisense RNA, produced either by the cleavage/polyadenylation of the chimeric intermediate or transcribed and separated from the sense RNA strand containing both 5'poly(U) and 3'poly(A), is unmodified but not for a long duration. It is, probably, very rapidly utilized as RdRp template and as rapidly separated (and modified, thus becoming undetectable, in the process) from still nascent
5'poly(U)-containing sense strand being transcribed from it. Alternatively, this duration could be even shorter, comprised only of the time period between the completion of the 3' polyadenylated antisense RNA synthesis and the commencement of separation/ modification starting at its newly added poly(A) as soon as a double-stranded 3'poly(A)/5'poly(U) structure, an initial helicase substrate, is generated. Thus, a temporal window of opportunity for the detection of an unmodified, and thus detectable, 3'poly(A)containing antisense RNA-derived "identifier" of the iPCR pathway is small, and, correspondingly, its steady-state level, is slight.

A temporal window of opportunity is much greater for the detection of an unmodified 5'poly(U)-containing sense RNA-derived "identifier" of Tier Two of mammalian mRNA amplification. After the commencement of the sense RNA synthesis starting with the 5'poly $(\mathrm{U})$, transcribed from the 3'poly(A) of the antisense RNA, the nascent sense RNA strand remains unmodified until its completion by the synthesis of the 3'poly(A) segment transcribed from the 5'poly(U) segment of the antisense RNA template, which is being modified and separated (unless it is being re-used as a template and therefore is already modified) by a helicase/modifying activity trailing the RdRp complex. Only when a nascent antisense RNA strand is being synthesized starting at the 3'poly(A) of the newly produced sense strand RNA, and the double-stranded 3'poly (A)/5'poly(U) structure is present, can the strand separation of and associated nucleotide modifications in the 5'poly(U)-containing sense RNA strand begin at its 3', the poly(A)-containing, end. And even then, the 5'poly(U)-containing "identifier" remains unmodified for the duration of the antisense RNA synthesis and strand separation/nucleotide modification downstream from it. Thus, once transcription of the sense RNA is initiated, starting with the 5 poly(U) and continuing into a truncated 5'UTR, the "identifier" is generated, and the time required for the completion of transcription, cumulatively with that needed for strand separation and associated nucleotide modifications downstream from the "identifier", provide a relatively large temporal window of detection opportunity. Consequently, at steady-state, there are many more unmodified, and therefore detectable by reverse-transcription based methods, 5'poly $(\mathrm{U})$ containing sense strand RNA-derived "identifiers" of the iPCR process than their 3'poly(A)-containing antisense strand RNAderived counterparts. It should be remembered, however, that the detectable, not yet modified, "identifiers" of the iPCR pathway represent only a very minor subpopulation of the products of Tier Two of mammalian mRNA amplification, with the bulk of its fully modified and thus undetectable products remaining for now, alongside with highly abundant chimeric RNA end product of Tier One, a "Dark Matter" RNA.

\section{The Two-Tier Paradox: Asymmetry of Outcomes}

Of the four Types of the chimeric pathway of mammalian RNA-dependent mRNA amplification described above, each constitutes a variant of Tier One of the mRNA amplification process, is defined by its translational outcome, and potentially can progress to Tier Two. Thus, the Type I of the chimeric pathway is characterized by the location of the ICE element of the antisense RNA strand within its segment corresponding to the 5'UTR of the conventionally produced mRNA. Consequently, the chimeric RNA end product, resulting from this Type of the chimeric Tier of amplification, contains the intact protein-encoding information of 
a conventional mRNA progenitor, and the translational outcome would be a polypeptide identical to the conventionally produced protein. If this Type of the chimeric pathway were to progress to Tier Two, the iPCR pathway, the functional outcome would be the same as in Tier One, namely the amplified sense strand RNA that retains a complete coding information content of a conventional mRNA and produces a polypeptide identical to the conventional mRNA-encoded protein. In the Type II of the chimeric pathway, the ICE is located within a segment of the antisense RNA strand corresponding to the coding region of a conventional mRNA and one of the possible outcomes is the chimeric RNA end product encoding a CTF of a conventional polypeptide. If this Type of the chimeric pathway were to progress to Tier Two of the mRNA amplification process, one may intuitively assume that the iPCRamplified sense RNA strand would also encode the same CTF as the chimeric RNA end product of Tier One and that, in general, any particular translational outcome of Tier One would carry on into Tier Two. This, however, is not the case because of the phenomenon of "Two-Tier Paradox".

The Two-Tier Paradox is defined as follows: Regardless of the translational outcome of the chimeric pathway Tier of mRNA amplification - a conventional polypeptide, a CTF, an unrelated polypeptide, and even an abortive translation or translational incompetence - the sense RNA strand produced in Tier Two, the iPCR pathway, would always retain the entire coding information of a genome-transcribed mRNA progenitor and would always direct translation of a polypeptide identical to its conventional mRNA-encoded counterpart.

As was mentioned above, whether Tier Two of mammalian RNA-dependent mRNA amplification, the iPCR process, does or does not occur is completely inconsequential for Tier One, the chimeric pathway. In turn, any particular translational outcome of Tier One does not affect in any way the translational outcome of Tier Two. This is because, in sharp contrast to and irrespective of any of the potentially multiple translational outcomes of the chimeric pathway, there is only one possible translational outcome of the iPCR process of mammalian mRNA amplification: A polypeptide identical to that produced by a conventional, genometranscribed mRNA progenitor of the amplification process. This discordance reflects the asymmetry in the truncation of sense and antisense constituent components of the chimeric intermediate during its cleavage at the conclusion of Tier One. The reason for this asymmetry is that the truncations of molecules in question are determined by two different, spatially independent, elements. The truncation of the sense strand component of the chimeric RNA end product is defined by the position of the ICE element whereas the TCE position determines the truncation of the antisense strand. Because the ICE can be anywhere in the antisense RNA molecule, so can the sense RNA strand's 5' truncation be, and because the TCE, by definition, can be only 3 '-terminal, the antisense RNA always loses only a short segment at its 3' end. This phenomenon is displayed in Figure 4. Whereas the sizes of the chimeric RNA end products are drastically different in steps $\mathbf{7}$ and $\mathbf{7}$ ', the sizes of the antisense RNA end products in the same steps are identical (the same is true for steps $\mathbf{E}$ and $\mathbf{E}$ ' of Figure 5). The 3'-truncated antisense RNA, provided it is 3 '-polyadenylated at the conclusion of Tier One, gives rise in Tier Two to the 5 'poly(U)-containing sense RNA strand 5'-truncated by exactly the same extent as seen in the 3 ' antisense RNA truncation, which in most, if not all, instances is substantially shorter than a typical 5'UTR of a conventional mammalian mRNA [88]. Consequently, the 5 ' poly(U)-containing sense RNA molecule, derived from the 3' poly(A)-containing antisense progenitor (the initial iPCR template) via the iPCR pathway, retains the bulk of its 5'UTR and the intact proteinencoding portion of a corresponding conventional mRNA. Therefore, regardless of the translational outcome of Tier One, even if it is an abortive translation or translational incompetence, in Tier Two the translational outcome would always be a polypeptide identical to that attained with a conventional mRNA amplification progenitor. The translational outcome of Tier Two would match that of Tier One only when the ICE is positioned within the antisense RNA segment corresponding to the 5'UTR of conventional mRNA progenitor. In all other cases, the outcomes can be quite remarkable. It is conceivable, for example, that the outcome of Tier One is an abortive translation or translational incompetence, yet this "failure" could be "rescued" by the Tier Two process, which would produce an intact conventional polypeptide encoded by an mRNA progenitor. No less remarkable could be cases where Tier One results in a CTF of a conventional protein whereas Tier Two would produce, in parallel, the intact conventionally encoded polypeptide. The possible $\beta$ APP-independent generation of beta-amyloid in Alzheimer's disease (discussed below) provides a good illustration of such a phenomenon. If the antisense $\beta A P P$ RNA strand, resulting from the cleavage of the chimeric intermediate and lacking only its TCE (or a fraction thereof), were 3'-polyadenylated in a cleavage-coupled manner, and utilized as the initial iPCR template in the second Tier of the mRNA amplification process, it would give rise to a 5'-truncated and poly $(\mathrm{U})$-containing sense strand retaining the bulk of the 5'UTR and the complete coding region of the conventional $\beta$ APP mRNA. Following its translation, the intact $\beta$ APP polypeptide would be generated alongside its beta-amyloid-carrying CTF, produced from the chimeric RNA with 5'-truncated coding region in the $\beta$ APPindependent manner, in the first, chimeric pathway, Tier of the mRNA amplification process. Even more striking is the possibility of a polypeptide unrelated to a corresponding conventionally produced protein and a translation product identical to it being generated concurrently by the chimeric pathway and by the iPCR process respectively.

\section{Reporter Constructs For The Chimeric Pathway Of Mammalian RNA-Dependent mRNA Amplification}

The availability of reporters for both Tiers of mammalian RNA-dependent mRNA amplification is essential for the further development of the field. To be effective, these reporter constructs should satisfy quite stringent requirements, namely to generate no "reporting" protein product (presuming that it is a protein, which does the "reporting") in the conventional gene expression process but to produce it only in the pathway the construct is designed to "report" on. To meet these requirements is not straightforward. Fortunately, a certain feature of the mRNA amplification process affords the opportunity to fulfill them. The feature in question is the manner of generation and processing of the chimeric intermediate that leads to both the loss of a portion of conventionally produced mRNA progenitor in the protein-encoding end products of both Tiers of mRNA amplification, and to the acquisition in the chimeric RNA end product of a component not present in the initial mRNA progenitor. This feature allows to implant in and/or 
to remove from the mRNA end products of amplification elements regulating the production of a "reporting" polypeptide.

Accordingly, a reporter construct for the chimeric pathway of mRNA amplification should be designed in such a way as to allow the expression of a "reporting" polypeptide only in this pathway, and to preclude it when the pathway is not in operation. Conceptually, there are two ways to do this. One approach (Type I) is to generate an RNA transcript eligible for the RNA-dependent amplification process and encoding a "reporting" polypeptide, say a tag peptide, but lacking an element required for its translation. Such transcript is "silent" and cannot be translated into a "reporting" protein product under conventional circumstances. However, if this RNA transcript is undergoing RNA-dependent mRNA amplification, an element needed for its translation can be provided by the antisense component of the chimeric RNA end product of amplification. Such design is based on the concept of "Type IV of the Chimeric mRNA Amplification Pathway" described above. Second approach (Type II) is to generate an amplification-eligible RNA transcript encoding a tag peptide and containing all necessary elements for its expression but also incorporating an upstream element preventing translation of the desired polypeptide. This inhibitory upstream element is then eliminated in the 5'-truncated sense RNA component of the chimeric RNA end product of Tier One of mRNA amplification. In both Types of construct design, the TCE and ICE elements are placed in close proximity to ascertain their anticipated interaction.

An example of the Type I of such reporter constructs, encoding a tag peptide that is produced only in the RNA-dependent mRNA amplification chimeric pathway, is shown in the upper panel of Figure 12. In the amplification-eligible RNA transcript, TAGPEPTIDE/UTR highlighted in blue denotes nucleotide sequence encoding a tag peptide followed by the 3'UTR and lacking the preceding AUG translation initiation codon, which is replaced with the ACA codon (highlighted in pink, as is its complement in the antisense RNA). There is also no functional translation initiation codon upstream from and in-frame with the tag peptide-encoding segment; no tag peptide can be translated from this RNA, it is translationally "silent" (line A). In the corresponding antisense RNA (line B), tagpeptide/utr, highlighted in blue, denotes antisense complement of the TAGPEPTIDE/UTR nucleotide sequence; it also contains the 3 '-terminal TCE and the internal ICE sequences (both highlighted in yellow). Moreover, the TCE contains the 5'aug3' codon (shown in 3' to 5' orientation and highlighted in green, as is its complement in the sense RNA strand) in optimal translation initiation context. During the antisense RNA folding into a self-priming configuration (line C), the "aug" codon in the TCE is accommodated by the "ugu", complement of the ACA codon highlighted in pink in the sense RNA. The design of the construct ascertains that the additional 3'-terminal " $\mathrm{C}$ " of the antisense RNA, not encoded in the DNA but a transcript of the 5'cap "G" of the sense-strand RNA [15-17], can be accommodated in the folded self-priming configuration (both 3'-terminal " $C$ " of the antisense RNA and matching "G" at the 5' end of the ICE are highlighted in gray). The self-primed structure is then extended, strands are separated and the cleavage occurs (red arrow) at the 5' end of the TCE (line D). In the chimeric RNA end product of amplification (line E), the TCE-derived "aug" codon, highlighted in green, is positioned, in optimal translation initiation context, upstream from and in-frame with the tag peptide-coding sequence and can initiate its translation. If the amplification process progresses to Tier Two, no tag peptide will be produced in the iPCR pathway, consistent with the Two-Tier Paradox principles (Tier Two translational outcome is identical to that attained with conventional mRNA). With this construct, a tag peptide is produced only if and when the chimeric mRNA amplification pathway is operational; its occurrence reports the activity of the pathway.

An example of the Type II of a reporter construct for the chimeric pathway of mammalian mRNA amplification is presented in the lower panel of Figure 12. mRNA transcribed from this construct (line $\mathbf{A}$ ) is eligible for RNA-dependent amplification and encodes a tag peptide (TAGPEPTIDE/UTR highlighted in blue denotes nucleotide sequence encoding a tag peptide followed by the 3 'UTR). The tag peptide-encoding segment is preceded by the in-frame AUG codon (highlighted in green, as is its complement in the antisense RNA) in optimal translation initiation context. Yet this tag peptide-coding information cannot be translated because upstream from it is located another AUG (highlighted in red, as is its complement in the antisense RNA), also in optimal translation initiation context but out-of-frame with the tag peptide-encoding nucleotide sequence, and it is this upstream AUG that will initiate translation and thus preclude production of the tag peptide. In the corresponding antisense RNA (line B), tagpeptide/utr, highlighted in blue, denotes antisense complement of the TAGPEPTIDE/ UTR nucleotide sequence. It contains the 3'-terminal TCE and the internal ICE sequences (both highlighted in yellow). Its folded configuration (line $\mathbf{C}$ ) can accommodate the additional 3'-terminal "C" of the antisense RNA, not encoded in the DNA but a transcript of the 5'cap "G" of the sense-strand RNA (both 3 '-terminal " $C$ " of the antisense RNA and matching " $G$ " at the 5' end of the ICE are highlighted in gray). The 3 ' terminus of the selfpriming structure is then extended, strands are separated and the cleavage occurs (red arrow) at the 5' end of the TCE (line D). If the upstream "inhibitory" AUG (highlighted in red) in the initial RNA transcript is placed within a segment of mRNA corresponding either to the TCE, or to the loop region of folded antisense RNA (as shown in the Figure), it would be lost in the chimeric RNA end product of amplification (line $\mathbf{E}$ ). The same result could be achieved by placing the upstream AUG within a segment of mRNA corresponding to the ICE element of the antisense RNA, but this could lead to complications stemming from a potential "chimeric junction shift" and generation of an additional upstream AUG codon in the chimeric RNA end product, and may require additional precautions. In this process, the resulting chimeric RNA end product retains only the AUG preceding, in-frame and in optimal translation initiation context, the tag peptide-encoding sequence. With such construct, a reporter peptide would be generated only when the RNA-dependent mRNA amplification process has been activated.

\section{Reporter Construct For Tier Two of Mammalian mRNA Amplification, The iPCR Pathway}

The design of a reporter construct for Tier Two of mammalian mRNA amplification, the iPCR pathway, is significantly more demanding and challenging. Not only should the tag peptide-encoding information in the initial RNA transcript be untranslatable, it should also remain untranslatable in the chimeric RNA end product of Tier One of the amplification process and yet 


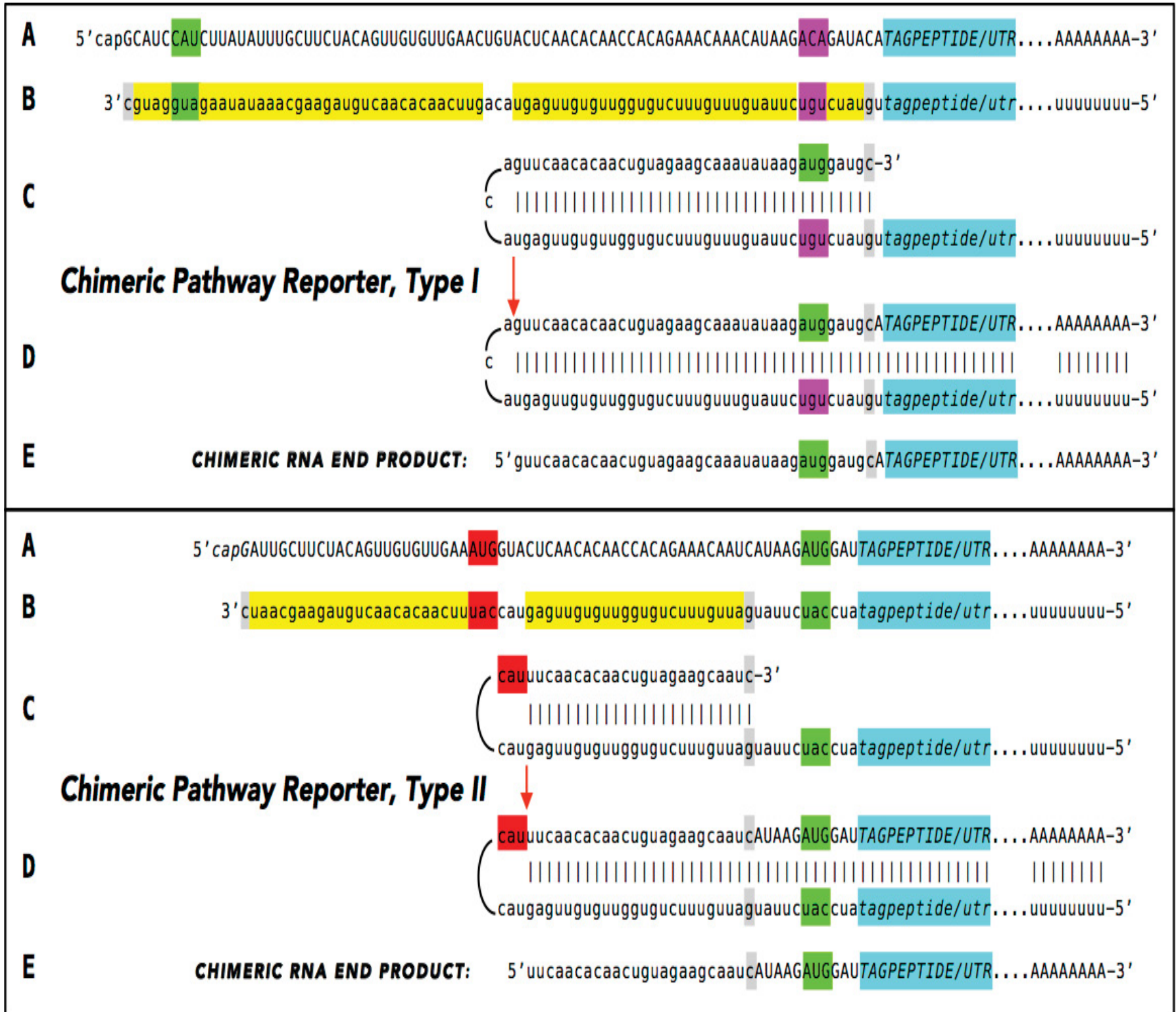

Figure 12: Design of RNA transcripts from reporter constructs producing a "reporting" polypeptide solely through Tier One, the chimeric mRNA amplification pathway. Uppercase letters: Nucleotide sequence of the sense RNA. Lowercase letters: Nucleotide sequence of the antisense RNA. TAGPEPTIDE/UTR, highlighted in blue, denotes nucleotide sequence encoding a tag peptide followed by 3'UTR; tagpeptide/utr, highlighted in blue, denotes complement of the TAGPEPTIDE/ UTR nucleotide sequence in the antisense RNA. Highlighted in green: 5'AUG3' in optimal translation initiation context or its complement on the opposite-oriented RNA strand. Highlighted in red: 5'AUG3' in optimal translation initiation context or its complement on the antisense RNA strand. Highlighted in yellow: the 3'-terminal complementary element, TCE, and internal complementary element, ICE, both on the antisense RNA. Highlighted in gray: 3'-terminal "c" of the antisense RNA not encoded in the DNA, a transcript of the cap"G" of mRNA, and matching "g" at the 5' end of the ICE on the antisense RNA. Red arrow: Position of cleavage of chimeric intermediate following strand separation. Top panel: A - Translationally silent RNA transcript encoding tag peptide and lacking the AUG translation initiation codon that was replaced with the ACA codon highlighted in pink, as is its complement in the antisense RNA (on the choice of the "ACA" see below); there are also no functional translation initiation codons upstream from and in-frame with the tag peptide-encoding segment. B - Antisense RNA; note that it contains the TCE and ICE elements and the 5'aug3' in optimal translation initiation context (shown as 3'gua5' and highlighted in green). C - Antisense RNA folded into self-priming configuration; note that the 5'aug3' is accommodated by the 3'ugu5' highlighted in pink, a complement of the "ACA" in the sense strand (the reason for its choice), and that the 3'-terminal "c", a transcript of the capG, is accommodated by the " $g$ " (both in gray). D - Extension of self- primed antisense RNA generates the chimeric intermediate containing covalently bound sense and antisense RNA strands; position of cleavage following strand separation is indicated by red arrow. $\mathbf{E}$ - Chimeric mRNA end product of mRNA amplification; note that the "aug" translation initiation codon in optimal translation initiation context (contributed by the antisense portion of the chimeric RNA) is in-frame with the tag peptide- encoding nucleotide sequence in the sense portion of the chimeric RNA end product and can initiate its translation. Note that no tag peptide can be produced via the iPCR process due to the lack of functional translation initiation codon in its sense RNA end product. Bottom panel: A - Tag peptideencoding segment in sense RNA strand is preceded in-frame by the AUG codon, highlighted in green, in optimal translation initiation context. However, tag peptide cannot be produced since this RNA contains an additional upstream "AUG" codon, highlighted in red (as is its complement in the antisense RNA), also in optimal translation initiation context, but out-of-frame with tag peptide-encoding nucleotide sequence. B - Antisense RNAcontaining the TCE and ICE elements. C - Antisense RNA folded into self-priming configuration; note that the 3'-terminal "c", a transcript of the capG, is accommodated by the " $g$ " (both in gray). D - Extension of selfprimed antisense RNA generates the chimeric intermediate; position of cleavage following strand separation is indicated by red arrow. $\mathbf{E}$ - Chimeric RNA end product of RNA-dependent mRNA amplification; the upstream AUG has been removed and the chimeric RNA contains only one "AUG" translation initiation codon, in optimal translation initiation context, preceding in-frame the tag peptide-encoding segment and capable of initiating its translation. 
it should be competent of translation into the tag peptide in the mRNA end product of the intracellular PCR pathway. Moreover, in addition to their evident complexity, these demands appear to fly in the face of the principle of Two-Tier Paradox, which posits that regardless of the translational outcome of the chimeric pathway Tier of mRNA amplification, the sense RNA strand produced in the iPCR pathway Tier would always retain the entire coding information of a genome-encoded mRNA and would direct the translation of a polypeptide identical to its conventionally encoded counterpart (described in the "Two-Tier Paradox" section above and further discussed below). Yet, however complex, these difficulties can be circumvented and the above demands satisfied in the following design.

In its initial part, this design exploits a concept utilized in the Type II of a reporter construct for the chimeric pathway of mRNA amplification, which is to generate an amplificationeligible RNA transcript that encodes a reporting polypeptide and contains all necessary elements for its expression but also includes an upstream element preventing translation of the desired polypeptide. This inhibitory upstream element is then removed in the 5'-truncated sense RNA component of the chimeric RNA end product, and an additional measure, described below, is used to prevent its translation into a tag peptide. As shown in Figure 13, mRNA transcribed from this construct (line A) is eligible for RNA-dependent amplification and encodes a tag peptide (TAGPEPTIDE/UTR highlighted in blue denotes nucleotide sequence encoding a tag peptide followed by the 3'UTR). The tag peptide-encoding segment is preceded by the in-frame AUU codon (highlighted in green, as is its complement in the antisense RNA; the choice of the "AUU" rather than "AUG" is explained below) that was shown to retain up to $67 \%$ efficiency of the AUG codon when in optimal translation initiation context [89], which is the case in this design. Yet this protein-coding information cannot be translated into a tag peptide because upstream from it is located another AUG (highlighted in red, as is its complement in the antisense RNA) in optimal translation initiation context and out-of-frame with the tag-peptide-encoding segment, and it is this upstream AUG that will initiate translation and prevent production of the tag peptide. In the corresponding antisense RNA (line B), Tagpeptide/utr, highlighted in blue, denotes antisense complement of the TAGPEPTIDE/UTR nucleotide sequence. It contains the 3'-terminal TCE and the internal ICE sequences (both highlighted in yellow). Its folded configuration (line $\mathbf{C}$ ) can accommodate the additional 3'-terminal "C" of the antisense RNA, not encoded in the DNA but a transcript of the 5'cap "G" of the sense-strand RNA (both 3'-terminal " $\mathrm{C}$ " of the antisense RNA and matching " $\mathrm{G}$ " at the 5 ' end of the ICE are highlighted in gray). The 3 ' terminus of the self-priming structure is then extended, strands are separated and the cleavage occurs (red arrow) at the 5' end of the TCE (line D). The upstream AUG in the initial RNA transcript is located within a segment of mRNA corresponding to the TCE element of the antisense RNA. Consequently, it is removed in the chimeric RNA end product of amplification (line E), which, unless additional measures are taken, would retain the AUU preceding in-frame the tag peptide-coding sequence that could be then translated.

As an "additional measure" to preclude translation of the chimeric RNA end product of Tier One, the 5'guu3' codon (highlighted in pink) is introduced in the antisense RNA in such position that in the folded molecule it matches the 3'uaa5', the complement of the AUU translation initiation codon on the initial RNA transcript. Accordingly, in the resulting chimeric RNA end product of Tier One, the tag peptide-encoding sequence is preceded by the "guu" codon, not known to initiate translation. There is also no functional translation initiation codon upstream from and in-frame with the tag peptide-encoding segment, and, therefore, it remains "silent", i.e. untranslatable. In this construct, the AUU translation initiation codon in the initial transcript was chosen because its complement, the 3'uaa5' (highlighted in green), is also complementary to the 5'guu3', which is used in this design to replace the AUU codon in the chimeric RNA end product of Tier One and thus to translationally incapacitate it. Such complementarity is important because, in this design, the TCE and the ICE elements should be fully complementary in order to ascertain that the cleavage of the chimeric intermediate occurs solely at the 5' end of the TCE element. If a TCE/ICE mismatch were allowed, the cleavage of the chimeric intermediate would occur at this mismatch. This would result in a sense/antisense "chimeric junction shift" if the 3-truncated antisense RNA retains a self-priming configuration and is re-used in the extension process (discussed in the "Processing of Chimeric Intermediates and Chimeric Junction Shift" section above). In such a case, during the extension of the self-primed 3'-truncated antisense RNA, the 3'uaa5' on the antisense RNA (highlighted in green) would be transcribed into the 5'AUU3' codon preceding in-frame and in optimal translation initiation context the tag peptide-encoding nucleotide sequence which would then become translatable in the chimeric RNA end product.

Meanwhile, whereas a tag peptide-encoding information remains untranslatable in both the initial RNA transcript and in the chimeric RNA end product of Tier One, the second end product of the chimeric pathway of mRNA amplification, the 3 '-truncated antisense RNA, retains the conventional complement, 3'uaa5' (highlighted in green), of the AUU translation initiation codon of the initial RNA transcript (line F). If this antisense RNA is 3'-polyadenylated in conjunction with the cleavage of the chimeric intermediate (line $\mathbf{G}$ ), the mRNA amplification process would progress into Tier Two, the iPCR pathway. In this process, the sense RNA strand would be transcribed from the antisense RNA template initiating within its 3'poly(A) segment (line $\mathbf{H}$ ); accordingly, the 3'uaa5' on the antisense RNA template would be transcribed into the AUU codon on the sense RNA strand. Separation of strands of such double-stranded structure would release the mRNA end product of Tier Two (line I; note that only one of potentially multiple iPCR cycles is shown in the Figure). It contains the tag peptide-encoding information preceded in-frame by the AUU codon in optimal translation initiation context and can be translated into a polypeptide "reporting" the occurrence of Tier Two of mammalian mRNA amplification, the intracellular PCR process.

As was mentioned above, the functional reporter construct for the iPCR pathway seemingly violates the principle of the Two-Tier Paradox. However, it does not; the principle remains valid. The Two-Tier Paradox was formulated $[8,15]$ for the defined systems where a 5'-terminal segment of an mRNA corresponding to the 3 '-terminal TCE element of the antisense RNA is always smaller than the 5'UTR of the same mRNA molecule. Considering that the average TCE could be 14-18 nucleotides long, as follows from our interpretation of the results described in $[16,23]$, this assumption 


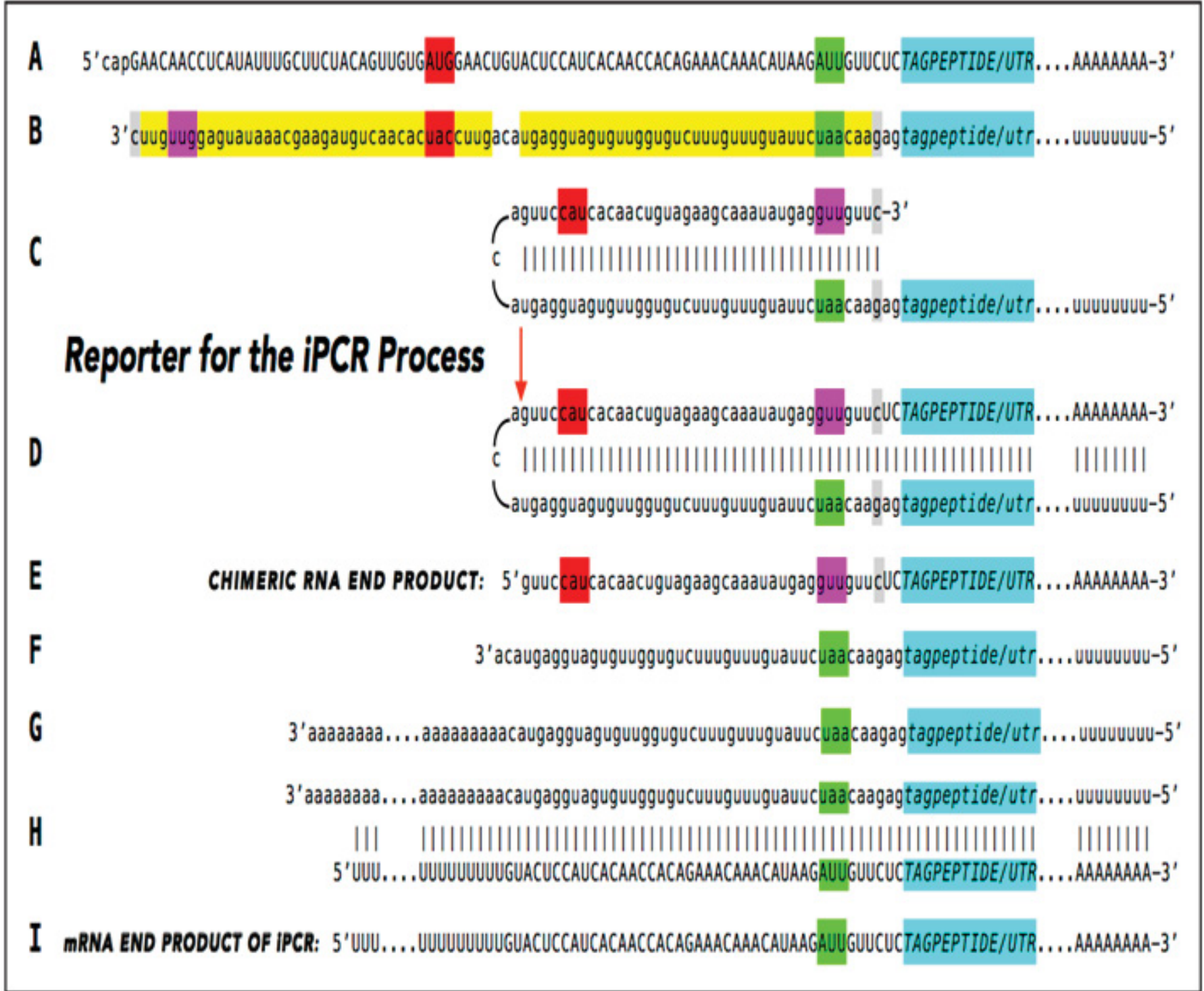

Figure 13: Design of RNA transcripts from reporter constructs producing a "reporting" polypeptide solely through Tier Two, the iPCR pathway of mRNA amplification. Uppercase letters: Nucleotide sequence of the sense RNA. Lowercase letters: Nucleotide sequence of the antisense RNA. TAGPEPTIDE/UTR, highlighted in blue, denotes nucleotide sequence encoding a tag peptide followed by 3'UTR; tagpeptide/utr, highlighted in blue, denotes complement of the TAGPEPTIDE/UTR nucleotide sequence in the antisense RNA. Highlighted in green: 5'AUU3' in optimal translation initiation context or its complement on the antisense RNA strand. Highlighted in red: 5'AUG3' in optimal translation initiation context or its complement on the antisense RNA strand. Highlighted in pink: 5'guu3' on the antisense RNA strand. Highlighted in yellow: the 3'-terminal complementary element, TCE, and internal complementary element, ICE, both on the antisense RNA. Highlighted in gray: 3'-terminal "c" of the antisense RNA not encoded in the DNA, a transcript of the cap"G" of mRNA, and matching " $\mathrm{g}$ " at the 5' end of the ICE on the antisense RNA. Red arrow: Position of cleavage of chimeric intermediate following strand separation. A - Tag peptide-encoding segment in sense RNA strand is preceded in-frame by the AUU codon, highlighted in green, in optimal translation initiation context and capable of initiating its translation. However, tag peptide cannot be produced since this RNA contains an additional upstream "AUG" codon, highlighted in red (as is its complement in the antisense RNA), also in optimal translation initiation context, but out-of-frame with tag peptide-encoding nucleotide sequence. B - Antisense RNA containing the TCE and ICE elements; note that it also contains the 5'guu3' highlighted in pink in its 3'-terminal portion. C - Antisense RNA folded into self-priming configuration; note that the 5'guu3' highlighted in pink is accommodated, via complementary interactions, by the 3'uaa5' highlighted in green, a complement of the AUU codonon the sense RNA strand (also highlighted in green) and that 3'-terminal "c", a transcript of the capG, is accommodated by the "g" (both in gray). D - Extension of self-primed antisense RNA generates the chimeric intermediate; position of cleavage following strand separation is indicated by red arrow. E - Chimeric RNA end product of RNA-dependent mRNA amplification. Note that the upstream AUG has been removed but the "AUU" translation initiation codon preceding in-frame the tag peptide-encoding segment in the initial RNA transcript has been replaced by the "guu" incapable of initiating its translation; there are no functional translation initiation codons upstream from and in- frame with tag-encoding segment, which remains translationally "silent". F - Second end product of the chimeric pathway of mRNA amplification, the 3'-truncated antisense RNA; note that it retains the conventional complement, 3'uaa5' (highlighted in green), of the AUU translation initiation codon of the initial RNA transcript. G - The 3- truncated antisense RNA is 3'-polyadenylated in conjunction with the cleavage of the chimeric intermediate; since it also contains the 5'-terminal poly(U) segment, it constitutes the initial template in the IPCR pathway of mRNA amplification. $\mathbf{H}$ - The sense RNA strand is transcribed from the antisense RNA template initiating within its 3'poly(A) segment. Note the 3'uaa5' highlighted in green on the antisense RNA template is transcribed into the AUU codon on the sense RNA strand. I - The mRNA product of the iPCR pathway released by strand separation (only one of potentially multiple iPCR cycles is shown). Note that it contains the tag peptideencoding information preceded in-frame by the AUU codon in optimal translation initiation context and can be translated into a polypeptide "reporting" the occurrence of Tier Two of mammalian mRNA amplification, the intracellular PCR process. 
appears to be correct for at least the decisive bulk of mammalian mRNA species. The above design of the reporter construct for the Tier Two pathway evades the restrains of the Two-Tier Paradox by having the upstream translation initiation codon positioned within the segment of the construct's RNA transcript corresponding to the TCE of the antisense RNA. In this setting, with regard to the upstream translation initiation codon, the TCE element is larger than the 5'UTR of mRNA; therefore rules of the Two-Tier Paradox do not apply. These rules, on the other hand, remain in effect with regard to the downstream translation initiation codon, the AUU, since for this codon the preceding 5'UTR of the mRNA transcript is larger than the TCE of its antisense RNA complement. Accordingly the translational outcome of the iPCR pathway is identical to that, which would be attained with the initial mRNA transcript if its translation were initiated from the AUU codon, consistent with the Two-Tier Paradox principle; the design, therefore, succeeds in separating regulatory elements into the Two-Tier Paradox rulesrelevant and -inapplicable.

\section{Malfunction Of Physiologically Occurring Mammalian RNA-Dependent mRNA Amplification As Basis Of A Disease: A Case Study}

The potential significance of RNA-dependent mRNA amplification in normal physiology, with every eligible genometranscribed mRNA molecule constituting a potential mRNA amplification progenitor, can hardly be overstated; in documented examples described above this process appears to be at the core of extremely high levels of production of globin chains in erythroid differentiation $[15,16]$ and of laminin chains in extracellular matrix deposition [17]. Malfunctions of this process may be involved in pathologies associated both with the deficiency of a protein normally produced by this mechanism and with the overproduction of a protein encoded by an mRNA species normally not involved in such a process. One of the experimental approaches to assess the involvement of mRNA amplification in pathologies of the first kind would be to interfere in vivo with the extent of complementarity between the two elements, the TCE and the ICE, involved in the antisense RNA self-priming, a core requirement for the progression of the amplification process. In fact, numerous experiments of this sort have been carried out by nature. One of the best opportunities to study the results of such "natural" experimentation is afforded by the occurrence of multiple types of beta-thalassemia. Beta-thalassemia is caused by the reduced or absent synthesis of the beta-globin chains of the hemoglobin tetramer and presents three clinical conditions, the beta-thalassemia carrier state, thalassemia intermedia, and thalassemia major. The beta-thalassemia carrier state results from heterozygosity for the disorder, is clinically asymptomatic and is defined by specific hematological features. Beta-thalassemia major is a severe transfusion-dependent anemia. Beta-thalassemia intermedia comprises a large group of disorders ranging from the mild to severe and heterogeneous at the molecular level. More than two hundreds beta-thalassemia-causing mutations have been identified. The majority of these mutations are single nucleotide substitutions, deletions and insertions. Rarely, there are manipulations of an oligonucleotide, usually leading to a frame shift, even more rare are deletions of the whole gene.

Four known single nucleotide mutations of interest to the subject matter under discussion occur within beta-globin gene segment corresponding to the 5'UTR of the human beta-globin mRNA [90]. They are the following: $+1 \mathrm{~A}>\mathrm{C},+10-\mathrm{T},+22 \mathrm{G}>\mathrm{A}$, and $+33 \mathrm{C}>\mathrm{G}$ (numbering from the transcription initiation site). None of these mutations interferes with translation of human beta-globin mRNA when compared with the wild type in cell-free translation assays. Each of these mutations reduces beta-globin production physiologically in vivo, causes mild beta-thalassemia in affected patients and appears to solely account for the disease. How can such an outcome be accomplished? Taking into account the established physiologically occurring phenomenon of globin mRNA amplification during erythroid differentiation, it is reasonable to venture that these mutations achieve their effect by interfering with the beta-globin mRNA amplification process; in such a case, the decreased production of beta-globin chains would result even if translation of beta-globin mRNA is completely unaffected. Using this assumption as the point of departure and considering that all four mutations are located within segment of the gene corresponding to the 5'UTR of mRNA, the most likely manner of interference is hindrance with the formation and function of self-priming structure on the beta-globin antisense RNA, a crucial stage in the mRNA amplification process. As diagrammed in Figure 14, this appears to be indeed the case. All four mutations evidently decrease the stability of the self-priming beta-globin antisense RNA structure. Moreover, there is more to it than the change in $\Delta \mathrm{G}$. Of the four mutations, three $(+10-\mathrm{T},+22$ $\mathrm{G}>\mathrm{A}$, and $+33 \mathrm{C}>\mathrm{G}$ ) destabilize the entire double-stranded blocks of nucleotides that they anchor, and one $(+1 \mathrm{~A}>\mathrm{C})$ weakens the interaction of the 3'-terminal " $\mathrm{C}$ " of beta-globin antisense RNA, essential for the commencement of the elongation process, with the complementary nucleotide within the self-priming structure. The destabilization of the self-priming antisense RNA structure results in decrease in the efficiency of the extension of the 3 ' terminus of the antisense RNA in particular and of the mRNA amplification process in general; less beta-globin mRNA is produced, less beta-globin polypeptide chains are generated, and the disease ensues. Thus, the results of the "natural" experiments confirm the predictable effects of the interference with and the malfunction of physiologically occurring mRNA amplification. The four classes of beta-thalassemia described above exemplify, therefore, the Type of diseases due to irregularities in the normally occurring mRNA amplification process.

\section{Abnormal mRNA Amplification-Mediated Overproduction Of A Protein: RNA- Dependent BAPP mRNA Amplification As Possible Driver of Alzheimer's Disease}

As was mentioned above, the activation of RNA-dependent mRNA amplification of mRNA species normally not involved in such a process could be at the root of diseases associated with the overproduction of a specific protein in general and of Alzheimer's disease in particular. At the beginning of the present article, in the section entitled "Pivotal Role of AUG encoding Met671 of $\beta$ APP" we proposed that in Alzheimer's disease, in addition to $A \beta$ derived in the $\beta A P P$ proteolytic pathway, beta-amyloid is generated in $\beta A P P-i n d e p e n d e n t$ manner by translation of severely 5 -truncated $\beta A P P$ mRNA where the first functional translation initiation codon is the AUG encoding Met671 of human $\beta A P P$ and contiguously preceding $A \beta$-coding nucleotide sequence. We also proposed that one of several mechanisms capable of generating such 5'truncated $\beta A P P$ mRNA is the $\beta A P P$ mRNA amplification pathway. If this 


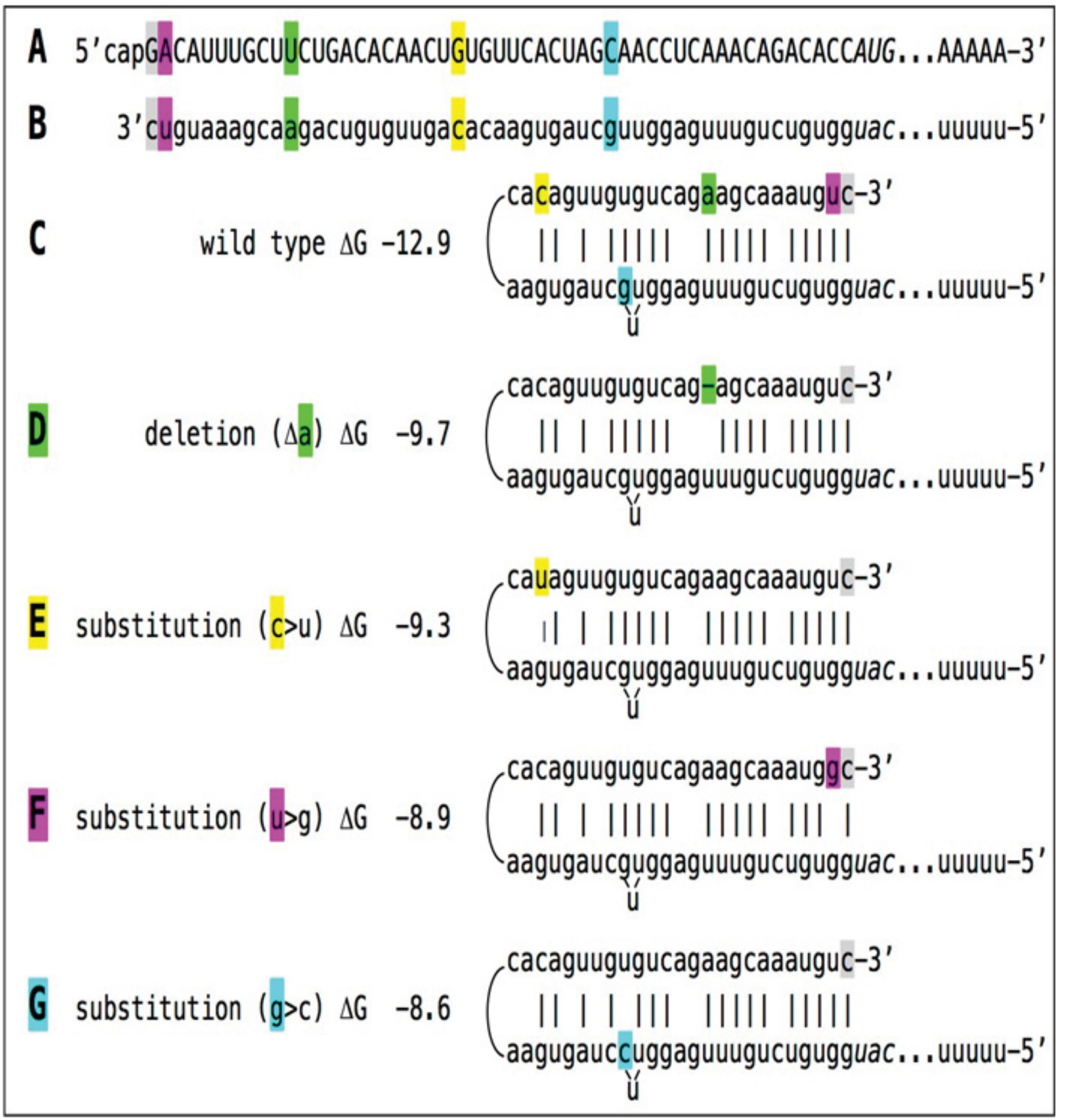

Figure 14: Beta-thalassemia-causing point mutations in the 5'UTR of human beta-globin mRNA: Projected mechanism of interference with the RNA-dependent mRNA amplification process. Uppercase letters: Nucleotide sequence of the sense RNA; lowercase letters: Nucleotide sequences of the antisense RNA. The "G" and the "c" highlighted in grey: Cap nucleotide in mRNA and its complement transcript in the antisense RNA respectively. The 5'AUG3' and the 3'uac5' in italics: Translation initiation codon in mRNA and its complement in the antisense RNA respectively. Highlighted in pink: "A" to "C" substitution in position +1 (counting starts at the TSS) of mRNA and corresponding substitution "u" to "g" in the antisense RNA. Highlighted in green: deletion of " $U$ " in position +10 of mRNA and corresponding deletion of "a" in the antisense RNA. Highlighted in yellow: "G" to "A" substitution in position +22 of mRNA and corresponding substitution "c" to "u" in the antisense RNA. Highlighted in blue: "C" to "G" substitution in position +33 of mRNA and corresponding substitution "g" to " $c$ " in the antisense RNA. Note that all mutations are in the 5'UTR of mRNA and in the corresponding segment of the antisense RNA. Line A: Nucleotide sequence of human beta-globin mRNA, wild type. Line B: Nucleotide sequence of beta-globin antisense RNA, wild type; note the 3'-terminal "c" highlighted in grey, a transcript of the capG of mRNA. Line C: Wild type antisense RNA folding into self-priming configuration; note that the 3'-terminal " $\mathrm{C}$ ", not encoded in the genome, is accommodated by the complementary "g". Lines D, E, F, and G: Folding into self- priming configuration of the antisense RNAs containing one of the mutations described above. Note that each mutation causes destabilization of self-priming structure; this destabilization, in turn, causes decrease of the efficiency of the elongation of the 3' end of the antisense RNA and of the effectiveness of the mRNA amplification process. Less beta-globin mRNA is produced in the mRNA amplification pathway, less beta-globin polypeptide chains are generated, and the disease ensues. 
process were indeed involved, it certainly would occur via the Type II chimeric mRNA amplification pathway. As described above, this Type of amplification is asymmetric in that the priming of the antisense RNA occurs within its segment corresponding to the coding region of mRNA. The generation of mRNA molecule where the AUG normally encoding Met671 of $\beta$ APP is the first translation initiation codon would necessitate a very substantial asymmetry: The AUG in question is situated over 2000 nucleotides downstream from the 5' terminus of $\beta$ APP mRNA; accordingly, the TCE and ICE elements of the corresponding antisense RNA molecule would be separated by about the same distance. The extent of projected TCE/ICE separation raises a legitimate question: Is such priming feasible?

\section{Projected Pathway of Asymmetric Amplification of Human} BAPP mRNA Resulting in Chimeric mRNA where the First Translation Initiation Codon Is the AUG Normally Encoding Met671 of $\beta$ APP

In general, whether an antisense RNA of interest contains topologically compatible TCE and ICE elements, whether these elements can form a self-priming structure and the TCE be extended, and, if the outcome is affirmative, what is the precise location of the ICE (the TCE is, by definition, always 3'-terminal) can be initially evaluated in a simplified model experiment. In such an experiment, an mRNA of interest serves as a template for synthesis of cDNA, initiating at the 3 '-terminal poly(A), and is subsequently removed by RNAse $\mathrm{H}$ activity present in a preparation of reverse transcriptase used (total RNA or total poly(A)-containing RNA can also be used, followed by identification and analysis of the product of interest). If an mRNA is fully transcribed, if complementary elements are present within the antisense strand (cDNA), if one of them is 3'-terminal, and if they are topologically compatible, i.e. mutually accessible within the folded antisense molecule, self-priming and the 3 ' end extension synthesis of a segment of the sense strand would occur. The junction between the antisense and sense components would define the site of self-priming and facilitate identification of the TCE and ICE elements.

Just such an experiment was, in fact, inadvertently carried out with human $\beta A P P$ mRNA [91]. The results of this experiment, "affirmative" in terms defined above, i.e., showing the occurrence of self-priming and the extension of the 3' terminus of the full-size $\beta A P P$ cDNA into a segment of the sense-orientation strand, but misinterpreted and eventually dismissed by the authors as an artifact [92], indicated the occurrence of the topologically compatible TCE and ICE elements within the antisense strand of $\beta$ APP mRNA and defined their nucleotide sequences as well as the position of selfpriming (i.e. the position of the ICE). Based on these results, the TCE/ ICE-guided folding and extension of the antisense strand of human BAPP mRNA [75-82] can be depicted as shown in Figure 15.

An approximately 30 nucleotide-long 3'-terminal segment of the antisense strand of human $\beta$ APP mRNA constitutes the TCE. Its counterpart, the ICE, is separated by nearly 2000 nucleotides, yet these elements \{both the TCE (top line in "a" and "b") and

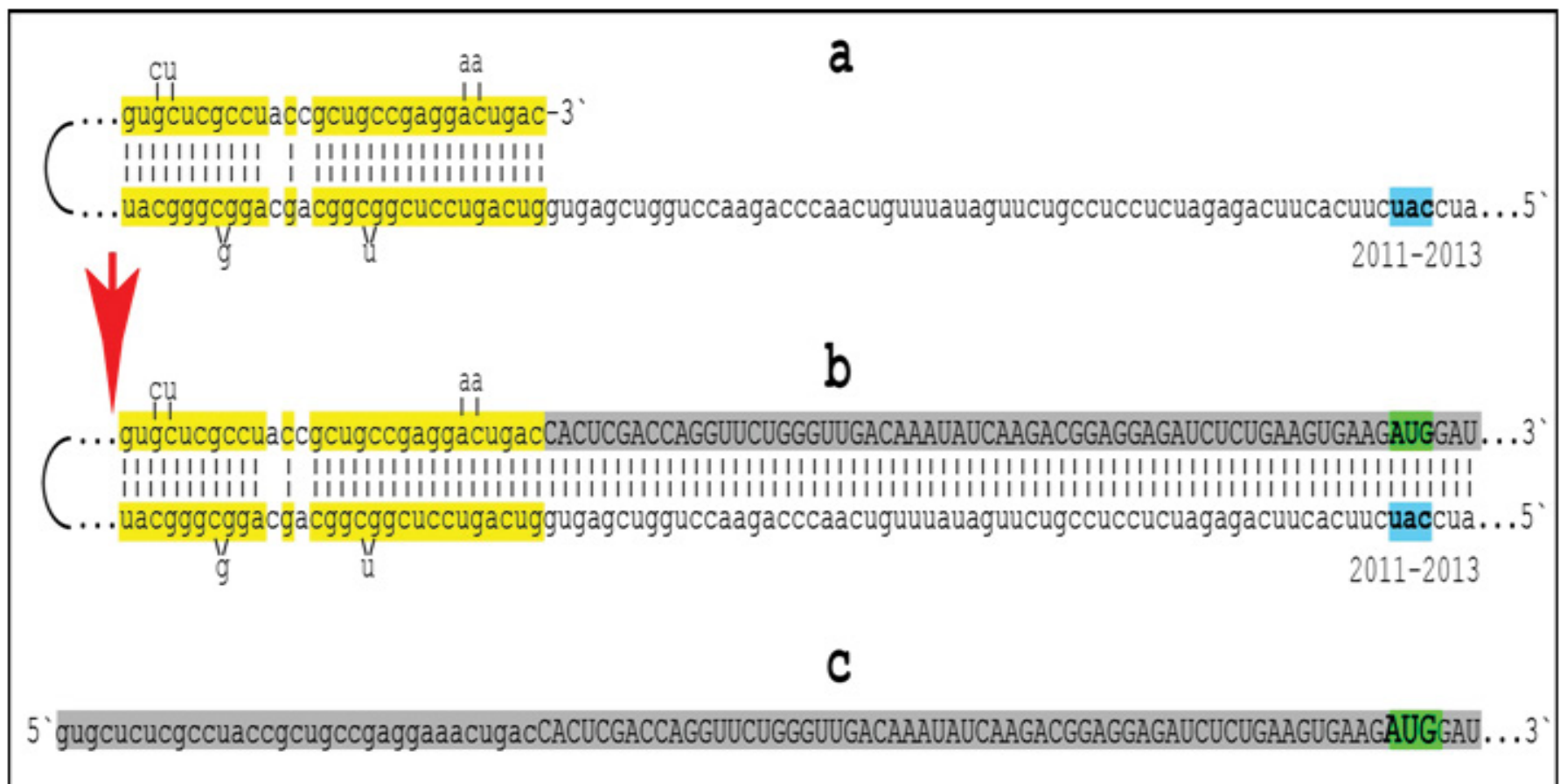

Figure 15: Projected topology of RNA-dependent generation of 5'-truncated mRNA encoding the C99 fragment of human beta- amyloid precursor protein. Lowercase letters: Nucleotide sequence of the antisense RNA. Uppercase letters: Nucleotide sequence of the sense RNA. Double-stranded portions highlighted in yellow: The TCE (top) and the ICE (bottom) elements of the antisense RNA. Note that the TCE and ICE are separated by about 2000 nucleotides. "2011-2013": Nucleotide positions on the antisense RNA (starting from the complement of the AUG encoding Met1 of the $\beta A P P$ ) of the "uac" (highlighted in blue) corresponding to the "AUG" (highlighted in green) encoding Met671 in the $\beta A P P$ mRNA. Panel a: TCE/ICE-guided folding of the $\beta A P P$ antisense RNA. 3'-terminal "c" corresponds to one of multiple transcription start sites of BAPP mRNA located 149 nucleotides upstream from its AUG initiation codon; note that such folding configuration would accommodate the additional 3'-terminal "C" (not shown), a transcript of the capG of $\beta A P P$ mRNA. Pane/ b: Extension of self-primed $\beta$ APP antisense RNA into sense RNA (highlighted in gray) and cleavage (red arrow; may also occur at one of the TCE/ICE mismatches) of the chimeric intermediate following strand separation. Pane/ c: Chimeric RNA end product (highlighted in gray) contains 5 '- terminal antisense segment extending into $\beta A P P$ mRNA truncated within its coding region. Its translation initiates from the "AUG" (highlighted in green and encoding Met671 in conventional $\beta A P P$ mRNA) immediately preceding the beta amyloid-encoding segment. 
the ICE (bottom line in "a" and "b"\} are highlighted in yellow in Figure 15) are topologically compatible and the folding of the antisense molecule results in a self-priming configuration capable of accommodating the additional 3'-terminal " $\mathrm{C}$ " not encoded in the genome, a transcript of the capG of $\beta A P P$ mRNA (the first nucleotide, "C", in the segment highlighted in grey in Figure 15b). The TCE serves as a primer and is extended; thus generating the sense strand as shown in Figure 15b. Strands are then separated as illustrated in Steps 5 and $\mathbf{6}$ of Figure 3, and cleavage occurs either at the mismatches within the TCE or immediately upstream of it as indicated by the arrow in Figure $15 \mathbf{b}$. The resulting chimeric RNA end product, shown in Figure 15c, consists of an antisense segment (the TCE or its portion) continued into a sense-orientated molecule. The translational outcome is decided by the first, 5'most, initiation-competent translation initiation codon. As shown in Figure $15 \mathbf{b}$ and $\mathbf{c}$, the first AUG codon (highlighted in green in Figure 15) is located 58 nucleotides downstream from the antisense portion of the chimeric RNA end product and it is, in fact, the AUG encoding Met671 in the intact $\beta$ APP mRNA! Translation from this position would produce the C-terminal fragment of $\beta$ APP containing beta-amyloid at its $\mathrm{N}$-terminus, the $\mathrm{N}$-terminal Met-containing C99 fragment of beta-amyloid precursor protein,

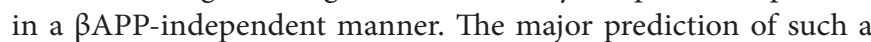
mechanism is a complete inefficiency of beta-secretase inhibition in Alzheimer's disease. This prediction was, in fact, born out in several massive stage III clinical trials [1,78]. Currently, there are no indications that Tier Two, the iPCR pathway, is operational in BAPP mRNA amplification in AD. But if it were employed, it could be expected that, as discussed above, alongside the C99 fragment produced in Tier One, the intact $\beta$ APP polypeptide would be generated, in parallel, as the translational outcome of Tier Two of $\beta$ APP mRNA amplification. Interestingly, if Tier Two of $\beta$ APP mRNA amplification, the iPCR process, were to occur, it would have no decisive effect on the progression of Alzheimer's disease because the bulk of its beta-amyloid output, generated in the $\beta A P P$ proteolytic/secretory pathway, would be secreted into the extracellular pool, in sharp contrast to the apparent intraneuronal retention of the $A \beta$ output of Tier One.

In Humans, Only a Subset of $\beta A P P$ mRNA Transcripts is Eligible for the RNA-Dependent mRNA Amplification Process: Transcription Start Site Usage May Contribute to Susceptibility or Resistance to AD

As was mentioned above, the presence of a regulatory element known as the "TATA-box" is characteristic for a large class of mammalian genes. Usually, it occurs about 25 nucleotides upstream from the Transcription Start Site (TSS) and rigidly defines its position. The mammalian $\beta$ APP gene belongs to a class of TATA-less genes that are characterized by multiple transcription start sites. There are at least five, and possibly more, positions where transcription of human $\beta A P P$ mRNA can be initiated $[75,93]$. Of those, only one, 149 nucleotides upstream from the AUG translation initiation codon, shown in Figure 15, results in an mRNA molecule eligible for the RNA-dependent mRNA amplification process, because only for this transcript would the position of the TCE element on its antisense strand be strictly 3 '-terminal $[75,93]$, and the additional 3'-terminal " $\mathrm{C}$ ", a transcript of the capG of $\beta$ APP mRNA [15-17], would be accommodated in the antisense RNA self-priming structure. Utilization of human $\beta$ APP transcription start sites can be, therefore, one of the factors that define susceptibility or resistance to Alzheimer's disease. The ability to regulate the usage of $\beta$ APP transcription start site(s), or even to shift it at will, could, therefore, open powerful therapeutic applications.

\section{Exclusivity of Alzheimer's Disease to Humans: Animal $\beta A P P$ mRNA is Ineligible for Amplification}

The presence of the poly(A) segment at the 3' terminus of an RNA makes the molecule an eligible RdRp template, but it does not necessarily make it eligible for amplification. For this, the antisense RNA strand should be capable of forming a stable self-priming structure in which its 3' terminus can be extended into a segment of the conventional mRNA molecule. Folding of the antisense RNA in such a self-priming configuration requires, in turn, the occurrence of two complementary and topologically compatible elements, one of which is strictly 3'-terminal. It appears that this requirement is not met in antisense RNA complements of $\beta A P P$ mRNA in non-human mammals. In studied animals, $\beta A P P$ antisense RNA segments corresponding to the TCE and ICE elements of human $\beta$ APP antisense RNA show little, if any, complementarity; moreover, the 3'-terminal segments of various animal antisense $\beta$ APP RNAs have no extensive complementarity with the rest of the molecule. Thus, in non-human mammals, $\beta$ APP mRNA, although a suitable RdRp template by virtue of containing the 3'-terminal poly(A), is not eligible for the RNA-dependent mRNA amplification process. Therefore, if, as suggested above, the extent of increase in beta-amyloid levels sufficient to trigger a cascade of molecular events culminating in AD cannot be reached by the $\beta$ APP proteolytic pathway alone and requires the activation of the $\beta$ APP mRNA amplification pathway, Alzheimer's disease can occur in humans but not in animals, a conclusion consistent with observations in the field. It should be mentioned that in light of the above considerations, the currently used $\mathrm{AD}$ models are inadequate for studies of the disease because they are lacking the $\beta A P P$ mRNA amplification pathway of beta-amyloid production, the major driver of the disease. The reason for this is not the lack of enzymatic apparatus but ineligibility of both, endogenous and transgenic $\beta$ APP mRNAs utilized in these models, for the RNAdependent amplification process [72-75], as well as the lack of means for accelerated activation of the enzymatic machinery of the mRNA amplification pathway [74,75]. Therefore, further development of the field requires generation of conceptually new experimental models. The proposed design of such models, as well as suggested methods of their construction, are discussed elsewhere [81,82]. Potentially optimal, neuronal culture-based, $\mathrm{AD}$ models are described in the "Research Strategies" section below.

\section{Activation of the BAPP mRNA Amplification Pathway in Alzheimer's Disease}

Above, we rationalized that in Alzheimer disease, the overproduction of beta-amyloid is effected by an $\mathrm{AD}$-specific $\mathrm{A} \beta$ production pathway and, moreover, that this pathway is independent from the $\beta$ APP proteolytic processing and that the output of this pathway is retained intraneuronally. The $\beta$ APP mRNA amplification pathway of beta-amyloid generation, discussed above, apparently fits this description since it is, evidently, completely independent from the $\beta$ APP proteolytic/ secretory process. The remaining outstanding questions are: How this pathway is activated and maintained in the disease and why the 
resulting beta-amyloid peptide, unlike its counterpart produced in the $\beta$ APP proteolytic pathway, is retained within neuronal cells rather than being secreted?

Recently, it was suggested [79-82] that in Alzheimer's disease, the $\beta$ APP mRNA amplification pathway in neuronal cells is triggered by mitochondrial dysfunction. Mitochondrial dysfunction in neurons is, in turn, caused by $\beta$ APP-derived $A \beta$ accumulated inside the cell to sufficient levels [79-82,94-110]. Whereas the scale of the postulated intraneuronal retention of beta-amyloid produced in the $\beta A P P$ mRNA amplification pathway is unparalleled in that it applies to the entire output or to the bulk of the output of the $\beta$ APP-independent $A \beta$ generation pathway, a notion of intracellular $\beta$ APP-derived $A \beta$, albeit as a fraction of its secreted counterpart, is not unprecedented. There are two potential sources of intracellular $A \beta$ produced in the $\beta$ APP proteolytic/secretory pathway. The more studied and understood source is cellular uptake of secreted extracellular beta-amyloid. Data obtained conclusively show that soluble extracellular $A \beta 42$ and $A \beta 40$ use endocytosis [111] to enter the cell and that $A \beta 42$ is taken up two times more efficiently than $\mathrm{A} \beta 40$ [112]. Beta-sheetrich $A \beta 42$ aggregates were observed to enter cells at low nanomolar concentrations [113]. In contrast, monomers were shown to bound to plasma membrane and to form aggregates there before cellular uptake and accumulation in endocytic vesicles [114], thus indicating that formation of $A \beta$ aggregates may be a prerequisite for cellular uptake [111,113-115]. Moreover, it was suggested that oligomer-specific $A \beta$ toxicity in cell models is mediated by its selective uptake [111]. Cellular uptake of $A \beta$ was also shown to be ApoE isoform-dependent and mediated by lipoprotein receptor LR11/SorLA [114]. ApoE4, a major genetic risk factor for AD, was shown to be much more efficient in mediating $A \beta$ uptake than ApoE3 and ApoE2 [114]. LRP, another member of the lipoprotein receptor family, binds to $A \beta$ directly or through ligands such as ApoE and undergoes endocytosis, thus facilitating cellular uptake of $A \beta$ [116]. The internalization of extracellular $A \beta$ can also be mediated by $\alpha 7$ nicotinic acetylcholine receptor [117-119], the scavenger receptor for advanced glycation, RAGE [120-122], the formyl peptide receptor-like 1, FPRL1 [123], and N-methyld-aspartate, NMDA, receptors [124]. A $\beta$ internalization was observed in multiple cell types, including neurons, astrocytes, glial cells and macrophages, where it appears to play different roles, and it occurs in cells of normal subjects as well as in cells of ADaffected individuals [125]. Because of its occurrence in healthy humans, this source of intracellular beta-amyloid appears to be insufficient alone to trigger mitochondrial dysfunction, $\beta$ APP mRNA amplification, and, consequently, AD.

The other potential source of intracellular $\beta$ APP-derived $A \beta$, operating only in beta-amyloid producing cells, i.e. neurons in the subject under discussion, is its retention within the cell, apparently essential for reaching critical levels sufficient to cause mitochondrial distress and trigger the disease. Whether $A \beta$ is retained intracellularly or is secreted into the extracellular pool is defined by the location at which the immediate beta-amyloid precursor, the C99 fragment, is cleaved by the gamma-secretase complex. The vast majority of $A \beta$ produced in the $\beta A P P$ proteolytic pathway is generated by cleavage at the plasma membrane and is secreted. However, gamma-cleavage can also occur in the Endoplasmic Reticulum (ER) [126], Golgi and trans Golgi network (TGN) [127], and at endosomal [126], lysosomal [126] and mitochondrial [128] membranes; such cleavages generate intracellularly retained $A \beta$. It has been shown that different isoforms of intracellular $A \beta$ can be generated at different locations. For example, cleavage within the ER produces predominantly A $\beta 42$ [129-133] whereas cleavage within the TGN mostly generates $A \beta 40$ [134]. Interestingly, these locations of intracellular $A \beta$ generation are limited to neurons [130]. Therefore, intracellular retention of $\beta$ APP-derived $A \beta$ is more likely to occur in neurons than in non-neuronal cells. Recent evidence suggests that subcellular localization of presenilin 2 (PSEN2) directs the assembly of gamma-secretase complex to specific cellular compartments and thus contributes to the balance between intracellular accumulation and secretion of $A \beta[112,135]$. Moreover, FAD-associated PSENs mutations were shown to pronouncedly augment the intracellular pool of $A \beta$ by determining localization and substrate specificity of gamma-secretase [135].

Whereas only a fraction of $A \beta$ produced in the $\beta A P P$ proteolytic pathway is retained intraneuronally, the entire output of the predominant $\beta$ APP mRNA amplification pathway of betaamyloid production, or at least the bulk or a substantial fraction of it, presumably remains inside the $\mathrm{AD}$-affected neurons (until they ultimately die as a result, that is). Normally, proteolytic processing of the bulk of $\beta$ APP occurs in the secretory pathway and culminates in gamma-secretase cleavage within the plasma membrane and subsequent secretion of newly generated $A \beta$ into the extracellular space. A nascent $\beta$ APP molecule is chaperoned into the secretory pathway through its $\mathrm{N}$-terminal signal peptide. There is, however, no N-terminal signal peptide in the translational end product of asymmetric amplification of human $\beta A P P$ mRNA. Indeed, the $\beta A P P$ mRNA amplification pathway results in the N-terminal Metcontaining C99 fragment, which lacks $\mathrm{N}$-terminal signal peptide and requires only gamma-secretase cleavage to produce $A \beta$ (or Met-A $\beta$ ). This cleavage can occur on intracellular membranes at a variety of sites, such as ER and TGN [127-132], where gammasecretase cleavage was shown to occur only in neurons [130], and even in mitochondria where all subunits of the gamma-secretase complex were shown to be present $[134,136,137]$. If this cleavage occurs in the ER, the $A \beta 42$ isoform will be predominantly produced [130]. If both ER and TGN sites are utilized, a mixture of $A \beta 42 /$ $A \beta 40$ will result [130]. For $A \beta$ to be retained intraneuronally, its precursor, the $\mathrm{C} 99$ fragment, produced either in the proteolytic pathway or in the AD-specific $\beta A P P$ mRNA amplification pathway, has to be processed, i.e. cleaved by gamma-secretase, on an intracellular membrane. In the $\beta$ APP proteolytic pathway this occurs infrequently, whereas in the $\beta A P P$-independent pathway of $\mathrm{A} \beta$ production, this is, apparently, the only, or at least the major, way. It should be mentioned that C99 fragment contains an internal trans-membrane domain, and when transiently expressed in non-neuronal cells without a signal peptide, it produces $A \beta$ that is secreted $[138,139]$. It appears, therefore that in neurons, $\mathrm{N}$-terminal Met-containing C99 produced in the $\beta$ APP mRNA amplification pathway is processed in a neuron-specific manner on the intracellular membranes [130] and thus is retained. In addition, if the heavily modified mRNA end product of RNA-dependent asymmetric human $\beta$ APP mRNA amplification, encoding the C99 fragment, is translated in a compartmentalized manner, this may also contribute to selection of gamma-secretase cleavage sites on intracellular rather than plasma membranes. If the chimeric $\beta$ APP mRNA amplification pathway were to progress into Tier Two of the amplification process, the impact of $A \beta$ produced in the iPCR 
pathway on the progression of the disease would be rather minor. This is because the iPCR pathway would produce the intact $\beta$ APP; following its proteolytic processing, the bulk of the resulting $A \beta$ would be secreted and thus "disabled" in terms of its capacity to cause mitochondrial dysfunction. On the other hand, because $\beta A P P$ mRNA product of the iPCR pathway would be heavily modified, it could be translated, and the resulting $\beta$ APP cleaved by beta- and gamma-secretases, in a compartmentalized manner on the intracellular membranes. In such a case, the resulting $A \beta$ would be retained intracellularly; this possibility remains to be investigated.

As for how the intraneuronal $A \beta$-initiated mitochondrial dysfunction activates the RNA-dependent mRNA amplification pathway, the missing links were provided in two recent studies $[73,74]$. In these investigations, it was shown that depolarization, a change in the electrical charge, which occurs during mitochondrial dysfunction, activates the protease OMA1, which is located on the inner of the two mitochondrial membranes surrounding the organelle. In turn, the activated OMA1 cleaves or facilitates the cleavage of another mitochondrial protein, DELE1, which resides in the space between the two mitochondrial membranes and is associated with the inner membrane, the locality of OMA1. Following the cleavage, a fragment of DELE1 is released to the cytosol where it binds to HRI and activates it. Activated HRI phosphorylates eIF2 $\alpha$; this leads to the integrated stress response. The ISR, as described above, suppresses global cellular protein synthesis but activates expression of selected transcription factors, notably ATF4, ATF5, and CHOP [66,67]. Some of these transcription factors either act as inducible components of the RdRp complex or enable their expression. Once the competent RdRp complex is assembled, the RNA-dependent mRNA amplification pathway is activated in neuronal cells (Figure 7). Importantly, as reasoned above, by the intrinsic logic of the process of ISR-activated mRNA amplification, the amplified mRNA has to be compatible with phosphorylated eIF $2 \alpha$ and freely translated under the ISR conditions, possibly due to its nucleotide modifications. This implies that the N-terminal Met-containing C99 fragment generated in the $\beta$ APP mRNA amplification pathway is produced via preferential translation from its amplified and thus modified mRNA template and, consequently, that intraneuronally retained $A \beta$ (or Met- $A \beta$ ) resulting from its processing, is further enriched (relative to the bulk of cellular proteins) in $\mathrm{AD}$-affected neurons under mitochondrial distress and the ISR conditions, thus augmenting the efficiency of the mRNA amplification process.

\section{Activation of the BAPP mRNA Amplification Pathway in "Swedish" FAD Mutants: A Case Study}

As an illustration of the concepts of activation of the $\beta$ APP mRNA amplification pathway discussed above, it is instructional to briefly review the case of the Swedish $\beta$ APP mutation. Swedish mutation is the first-discovered $\beta A P P$ mutation resulting in early onset Alzheimer's disease (familial AD). It affects amino acid residues 670 (normally lysine) and 671 (normally methionine) of $\beta A P P$ and replaces them with asparagine and leucine respectively [140]. These two residues are immediately adjacent to the site of beta-secretase cleavage, which occurs between positions 671 and 672 in both wild-type and the Swedish $\beta$ APP mutant. Therefore, it was initially assumed that the Swedish mutation causes the disease by increasing the efficiency of $\beta$ APP cleavage by beta-secretase and thus elevating the production of $\mathrm{A} \beta[140,141]$. This is, however, not the case; the efficiency of beta-cleavage of the Swedish $\beta$ APP mutant is similar to that of wild-type $\beta A P P$. And yet, the Swedish $\beta A P P$ mutation reliably and uniformly triggers early onset Alzheimer's disease in its carriers. The proffered interpretation, that in this case the beta-cleavage outcompetes the alpha-cleavage of $\beta A P P$ and thus increases the production of $A \beta$ [141], appears insufficient and necessitates an alternative explanation.

In the framework of the $\beta A P P$ mRNA amplification theory of Alzheimer's disease, where the emergence of symptomatic AD is equated with the activation of the $\beta A P P$ mRNA amplification pathway, it can be assumed that the occurrence of the Swedish mutation substantially accelerates the commencement of $A \beta$ generation in the mRNA amplification pathway. In this respect, it could be argued that the operation of the $\beta A P P$ mRNA amplification pathway of $A \beta$ production is inconsistent with the Swedish mutation because the mutant $\beta$ APP mRNA no longer encodes Met671, postulated to initiate translation of the amplified $\beta A P P$ mRNA. This argument, however, would not be valid for the following reason. In wild-type $\beta A P P$ mRNA, the nucleotide sequence encoding residues 670-672 is: AAG AUG GAU. In the Swedish mutant this sequence is changed to: AAU CUG GAU. The CUG triplet, which replaces the AUG in the mutant $\beta$ APP mRNA, was shown to initiate translation with $82 \%$ efficiency of the AUG when it is situated in the optimal translation initiation context [89]. It is in the Swedish $\beta A P P$ mRNA mutant (the A in position -3 and the $\mathrm{G}$ in position +4 relative to the $\mathrm{C}$ of the CUG), and thus would be operational in the initiation of translation.

Therefore, if and when activated, the generation of $A \beta$ via the $\beta$ APP mRNA amplification pathway would be (almost) perfectly efficient in cells carrying the Swedish mutation. The question is how the occurrence of this mutation accelerates activation of the $\beta$ APP mRNA amplification pathway? The generic answer is: Location, location, location. In this particular case, it is the location of $\beta$ APP cleavage by beta- and gamma-secretases. Whereas in wild-type the beta-cleavage occurs in the endosomal compartments [142] and leads, eventually, to secretion of $A \beta$, in Swedish mutation carriers it takes place to a large extent in the Golgi complex [140,141], where gamma-secretase was also shown to be present and gammacleavage to occur [127]. It follows that in Swedish mutation carriers, a substantial fraction of $A \beta$ produced by $\beta$ APP proteolysis would be retained intracellularly because gamma-secretase cleavage of C99 occurs on intracellular membranes. Initially, however, it was speculated [141] that the processing of $\beta$ APP containing the Swedish mutation occurs not in the Golgi complex itself but in Golgi-associated secretory vehicles, and, therefore, it culminates in secretion of the resulting $A \beta$. Subsequent investigations indicated that this is not the case. Indeed, the notion of intracellular retention of beta-amyloid generated by the proteolytic processing of $\beta$ APP containing the Swedish mutation was validated experimentally. It was shown [143] that a significant portion of $\beta$ APP containing the Swedish mutation is processed in a pathway, which is distinctly different from the conventional $\beta$ APP secretory proteolytic process (that occurs in a post-Golgi compartment). Not only is it processed in the Golgi complex itself, but the processing also results in the intracellularly retained $A \beta$ and, consequently, in its rapid accumulation within the cell [143]. Thus, what is accelerated (or, rather, substantially shortened) in carriers of the Swedish $\beta$ APP mutant is the duration of $A \beta$ accumulation to critical intraneuronal 
levels sufficient to trigger mitochondrial dysfunction, to initiate the ISR and to activate the $\beta$ APP mRNA amplification pathway and elicit symptomatic manifestation of the disease. Consequently, in Swedish mutation carriers, symptomatic AD uniformly ensues much sooner (early onset) than in sporadic late onset cases seen in wild-type $\beta$ APP carriers where it takes most of the lifetime to build up sufficiently high levels of intracellular $A \beta$.

\section{The Engine that Drives Alzheimer's Disease}

To summarize the preceding sections, the intraneuronal accumulation of $A \beta$, conventionally produced in the $\beta$ APP proteolytic pathway to levels sufficient to trigger mitochondrial dysfunction and, consequently, activation of the $\beta$ APP mRNA amplification pathway, plays the role of a starter motor in getting car engine moving in a self-sustainable manner. In this context, importantly, the rate of intraneuronal retention of $\beta$ APP-derived beta-amyloid, combined with that of its uptake, apparently plays the decisive role in defining the susceptibility to AD. The "Starter Motor", once switched on, activates the "Engine" that drives Alzheimer's disease. Indeed, mitochondrial distress leads to activation of the mitochondrial protease OMA1 followed by the cleavage, still within the mitochondria, of DELE1, release of one of DELE1 fragments into the cytosol, its binding to and activation of the HRI kinase, consequent phosphorylation of eIF $2 \alpha$, and commencement of the integrated stress response. Within the framework of the ISR, it also leads to the activation of the RNAdependent mRNA amplification machinery and, provided $\beta$ APP mRNA is eligible, of the predominant, $\mathrm{AD}$-specific, pathway of $\mathrm{A} \beta$ generation, the $\beta A P P$ mRNA amplification process [79-82]. The entire output of this pathway, or at least the bulk or a substantial fraction of it, is retained intraneuronally. Drastically increased levels of intracellular $A \beta$ promote, in turn, further mitochondrial dysfunction, the OMA1 to DELE1 to HRI signaling pathway is maintained, and the integrated stress response and, consequently, the $\beta A P P$ mRNA amplification process, are sustained, thus generating self-perpetuating beta-amyloid overproduction/ mitochondrial dysfunction mutual feedback cycles, which constitute the $\mathrm{AD}$ "Engine". These relationships are presented diagrammatically in Figure 16. It depicts the mutual feedback cycles as a two-stroke "Engine", the "Engine" that drives betaamyloid overproduction and, consequently, Alzheimer's disease; ultimately, it triggers neuronal death. To develop sporadic AD, it takes, apparently, a lifetime of intraneuronal accumulation of conventionally produced $A \beta$ to critical levels sufficient to trigger mitochondrial dysfunction, the integrated stress response and, consequently, the RNA-dependent mRNA amplification pathway, combined with certain susceptibility factors (e.g. appropriate TSS usage permitting the operation of $\beta A P P$ mRNA amplification). In familial AD, because of abnormal $\beta$ APP proteolysis and/or other factors, critical intraneuronal levels of conventionally produced $\mathrm{A} \beta$, or of its more "toxic" isoforms with increased capacity to aggregate and to trigger mitochondrial dysfunction, are reached sooner and the disease occurs earlier in life.

Dynamics of AD in the New Paradigm: Alzheimer's Disorder is a "Fast" Disease Preventable by Therapeutic Intervention Initiated Even Late in Life, and Treatable at the Symptomatic Stages

The New Paradigm theory of AD posits that Alzheimer's disorder is a "fast" disease $[81,82]$. This is in sharp contrast to a view, prevailing until recently, that $\mathrm{AD}$ is a quintessential "slow" disease, which develops throughout the life as one prolonged process. Dynamics of the disease in these two Paradigms is presented in Figure 17. Its interpretation in the Old Paradigm is shown in panels $\mathbf{A}$ and $\mathbf{B}$. This dynamics is single-phased and can be divided into asymptomatic (red lines) and symptomatic (red blocks) portions. A $\beta$, it is assumed, is overproduced (and secreted) solely in the $\beta$ APP proteolytic/secretory pathway. As its extracellular levels increase, it triggers neurodegeneration (red lines) starting early in life. Damages accumulate and manifest symptomatically (red blocks) late in life in sporadic cases (panel A). In familial $\mathrm{AD}$ cases, where mutations in the $\beta \mathrm{APP}$ gene or in presenilins increase production of either common $A \beta$ isoform or of its more toxic isoforms, neurodegeneration reaches critical threshold sooner and AD symptoms occur earlier in life, mostly in the late 40s and 50s (panel B). In this Paradigm, the disease is considered untreatable in the symptomatic phase and there are currently no preventive $\mathrm{AD}$ therapies, but if they were available, according to this viewpoint, it would be largely futile to intervene late in life in SAD case or at mid-age in case of FAD because, although $\mathrm{AD}$ symptoms have not yet manifested, the irreversible damage has already occurred during the preceding decades. In the Old Paradigm, to be effective, preventive therapeutic intervention should be initiated early and continued for life.

Dynamics of the disease in the New Paradigm, illustrated in panels $\mathbf{C}(\mathrm{SAD})$ and $\mathbf{D}(\mathrm{FAD})$ of Figure 17, is radically different [80-82]. This dynamic is biphasic. In the first phase, only the $\beta A P P$ proteolytic pathway of $A \beta$ production is in operation. This phase is a slow process of intraneuronal beta-amyloid accumulation. It occurs via cellular uptake of secreted $A \beta$ and the intracellular retention of a fraction of $\beta$ APP-derived $A \beta$. These processes are common to Homo sapiens, including healthy humans, and to non-human mammals, and result neither in noticeable damage (black lines), nor in any manifestation of the disease; there is, in fact, no disease in this phase. The second phase occurs exclusively in humans and commences with the intraneuronal $\mathrm{A} \beta$-induced mitochondrial dysfunction, which, in turn, mediates the activation of the $\beta$ APP mRNA amplification pathway shortly before symptomatic onset of the disease. In this phase, the rate of production and the extent of intraneuronal accumulation of retained $A \beta$ sharply accelerate, causing, after a lag period when amplified Met-C99-encoding RNA accrues and intraneuronal A $\beta$ further accumulates (black lines), significant neurodegeneration (red lines), and triggering $\mathrm{AD}$ symptoms (red blocks); this phase is fast. In this Paradigm, a preventive therapy for $\mathrm{AD}$, an $\mathrm{AD}$ "statin", would be effective when initiated at any time prior to the commencement of the second phase (panel E). Accordingly, one of conceivable preventative therapeutic approaches is the suppression of mitochondrial distress; this would preclude or interrupt the mutual feedback cycles discussed above and expectantly block the activation of or disable the "Engine" that drives the disease. The feasibility of such strategy is supported by a recent study with C. elegans [144]. In this investigation, the neuronal beta-amyloidinduced mitochondrial dysfunction was modeled on $\mathrm{AD}$-related observations and achieved by expressing human A 342 specifically in neurons (GRU102). Significantly, treatment with an anti-diabetes drug, metformin, reversed $A \beta$-induced metabolic mitochondrial defects and normalized lifespan of GRU102 that otherwise was substantially reduced, thus establishing metabolic mitochondrial 

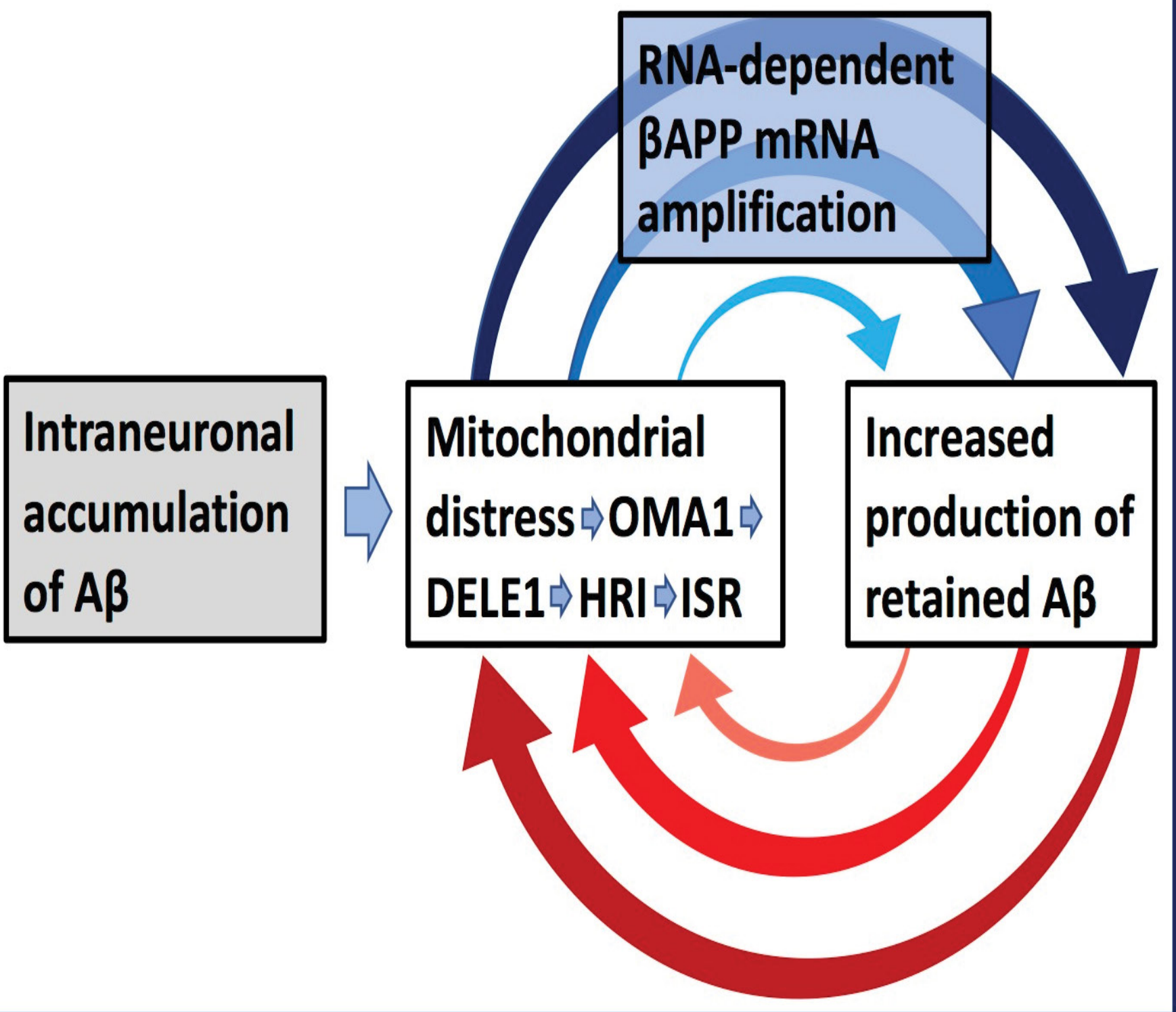

Figure 16: The "Engine" that drives AD: Self-propagating mutual feedback cycles of mitochondrial dysfunction-mediated overproduction of $A \beta$ and vice versa in Alzheimer's disease. Highlighted in grey: "Starter Motor" - Intraneuronal accumulation of A $\beta$ via both cellular uptake of secreted peptide and retention of a fraction of beta-amyloid produced in the $\beta A P P$ proteolytic pathway; Box highlighted in blue: Asymmetric RNA-dependent $\beta A P P$ mRNA amplification resulting in mRNA encoding the Met-C99 fragment of $\beta A P P$, a molecular basis of A $\beta$ overproduction in Alzheimer's disease. Large horizontal arrow: Life-long intraneuronal accumulation of $A B$ to critical levels triggers mitochondrial dysfunction and acts as a "Starter Motor", which initiates the self-sustainable "Engine" that drives AD. Arched arrows: Self-propagating mutual feedback cycles; Blue arches: Mitochondrial dysfunction-caused depolarization activates OMA1, which cleaves or facilitates the cleavage of another mitochondrial protein, DELE1; a fragment of DELE1 is released to the cytosol where it binds to HRI and activates it. Activated HRI phosphorylates elF2 $\alpha$, the ISR commences, the bulk of cellular protein synthesis is suppressed, select transcription factors are expressed, inducible components of the RdRp complex are produced, and the mRNA amplification pathway is activated, with the entire output of A $\beta$ being retained intraneuronally. Red arches: Drastically increased levels of intraneuronal $A \beta$ promote, in turn, further mitochondrial dysfunction; the OMA1 to DELE1 to HRI signaling pathway is preserved, the integrated stress response is sustained, and the BAPP mRNA amplification process is maintained, thus generating self-perpetuating beta-amyloid overproduction/mitochondrial dysfunction mutual feedback cycles.

dysfunction as a promising and viable therapeutic intervention target in Alzheimer's disease. Moreover, there are good reasons to believe that with a drug interrupting mutually perpetuating $\beta$ APPindependent $A \beta$ production/mitochondrial dysfunction cycles, the "Engine" that drives Alzheimer's disease, it would be possible not only to preempt the disease but also to stop and to reverse, i.e. to cure it even when early AD symptoms have already manifested. This assumption is based on observations made in studies where
$\mathrm{AD}$ symptoms, as well as neurodegeneration, were reversed by suppressing, via BACE inhibition, overproduction of betaamyloid in mouse AD models generating $\mathrm{A} \beta$ solely in the $\beta \mathrm{APP}$ proteolytic pathway $[145,146]$. The New Paradigm of Alzheimer disease establishes the feasibility of prevention of and interference with the disease and suggests an array of novel therapeutic targets described below. Most importantly, after years of despair in the field, it makes a case for optimism [82]. 


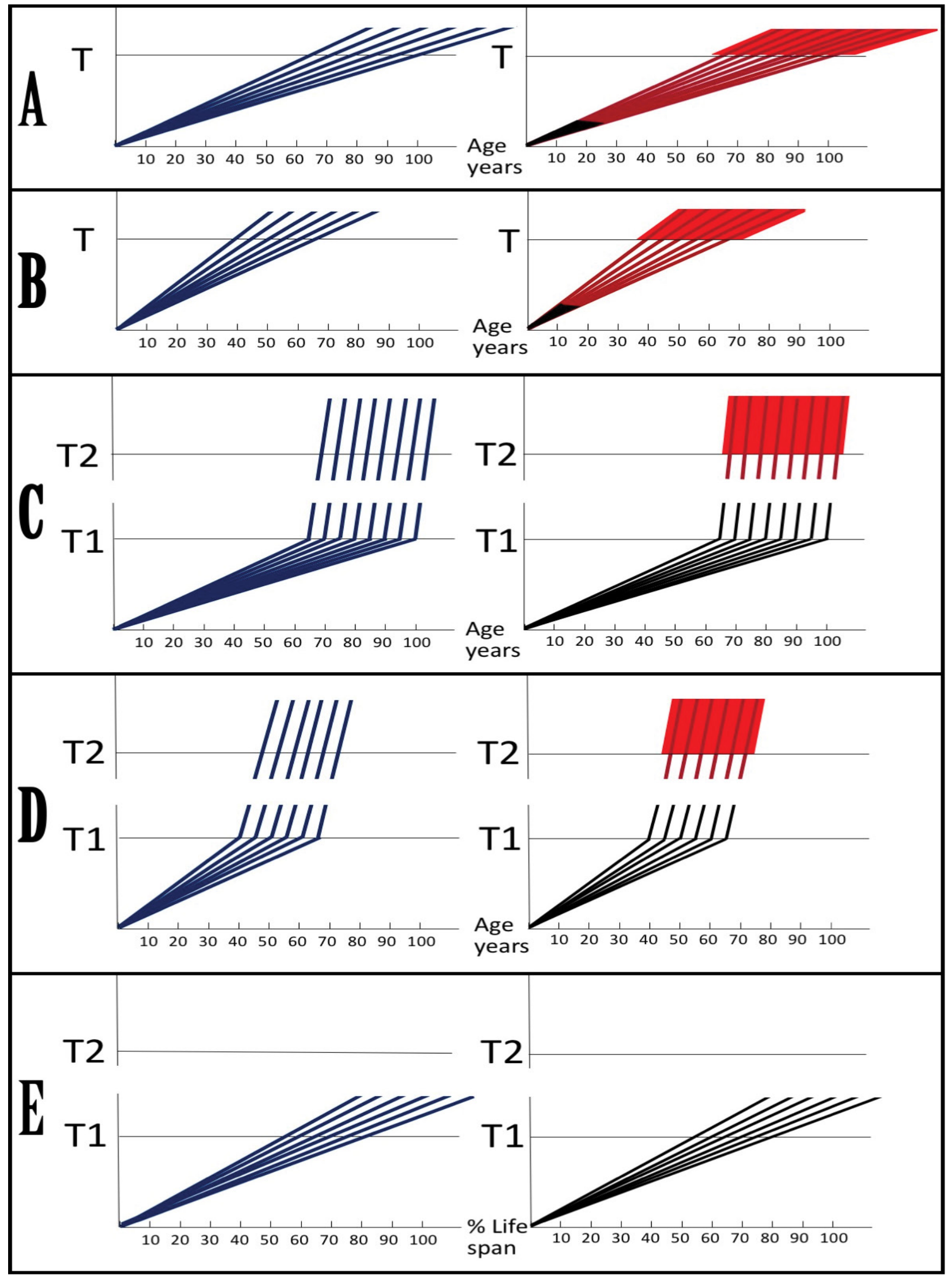


Figure 17: Dynamics of Alzheimer's disease in two Paradigms. Left panels: Dynamics of A production; Right panels: Dynamics of neurodegeneration. Blue lines: Levels of beta-amyloid; Red lines: Extent of neurodegeneration; Black lines: Indicator lines, no noticable neurodegeneration; Red blocks: Symptomatic manifestation of AD. T: Threshold of symptomatic manifestation of AD (reflects levels of extracellular $A \beta$ and assumed consequent extent of neurodegeneration); T1: Threshold of activation of RNA-dependent $\beta$ APP mRNA amplification (reflects intraneuronal A $\beta$ levels and the consequent extent of $A \beta-$ coupled mitochondrial dysfunction); Numerous genetic factors such as the occurrence of various alleles of ApoE gene, as well as certain epigenetic factors, influence the age when thresholds $T$ and $T 1$ are reached, hence, the fanning lines. T2: Threshold of symptomatic occurrence of AD (reflects levels of intraneuronal A 3 degree of mitochondrial dysfunction and consequent extent of neurodegeneration). Panels $\mathbf{A}, \boldsymbol{B}$ : View of the dynamics of $A D$ in the Old Paradigm (A: Dynamics of SAD; $B$ : Dynamics of FAD). Levels of extracellular $A \beta$ increase, neurodegeneration starts early and accumulates throughout the life. When threshold $T$ is reached, AD symptoms manifest. Panels $\boldsymbol{C}$, $\mathbf{D}$ : The outlook on the dynamics of $A D$ in the New Paradigm (C: Dynamics of SAD; $\mathbf{D}$ : Dynamics of FAD). Levels of intraneuronal $A B$ increase, the extent of mitochondrial dysfunction reaches threshold T1, the integrated stress response is triggered, and RNA-dependent BAPP mRNA amplification is activated. There is no noticeable neurodegeneration until after a lag period (when amplified RNA encoding the C99 fragment of $\beta A P P$ accrues and intraneuronal $A \beta$ further accumulates) following the crossing of T1 threshold and activation of $\beta A P P$ mRNA amplification; when the extent of neurodegeneration reaches threshold T2, AD symptoms manifest. Panel E: Dynamics of A $\beta$ production and neurodegeneration in non-human mammals and in humans not susceptible to AD or successfully treated: T1 threshold is crossed but BAPPP mRNA is not amplified in animals because it is not eligible for RNAdependent amplification process. There is no noticeable neurodegeneration; T2 threshold is not reached, no AD symptoms manifest, no disease occurs. Note: Scenario depicted in panel (E) would occur in humans not susceptible to Alzheimer's disease due to variations in $\beta A P P$ TSSs utilization or for other reasons, or when the $\beta A P P$ mRNA amplification pathway of $A \beta$ production is effectively suppressed by therapeutic intervention.

\section{Alternative Mechanisms of Generation Of 5'-Truncated BAPP mRNA Where The First Translation Initiation Codon Is The AUG Normally Encoding Met671 Of BAPP}

The reason why RNA-dependent mRNA amplification was suggested above as the likely mechanism underlying the generation of 5'-truncated $\beta$ APP mRNA, where the first translation initiation codon is the AUG normally encoding Met671 of $\beta A P P$, is its plausibility. Indeed, as described in detail above, the mRNA amplification pathway was shown to be associated with the overproduction of specific proteins in other systems; the integrated stress response, potentially responsible for the activation of the RdRs machinery, is clearly elicited in AD-affected cells via OMA1 - DELE1 - HRI axis of mitochondrial distress; finally, human $\beta A P P$ mRNA appears to be eligible for precisely the type (Type II) of mRNA amplification capable of generating suitably 5 '-truncated mRNA end product. The compendium of these factors makes the involvement of the mammalian RNA-dependent mRNA amplification pathway in generation of $\mathrm{A} \beta$ in $\mathrm{AD}$ highly probable.

There are, however, other cellular mechanisms capable of generating 5'-truncated $\beta$ APP mRNA with the AUG normally encoding Met671 of $\beta$ APP being the first functional translation initiation codon. The initiation of translation from this particular AUG codon appears to be one common feature of all mechanisms capable of $\beta$ APP-independent production of $\mathrm{A} \beta$. The other postulated common feature is the intraneuronal retention of the entire output, or of the bulk or at least of a substantial fraction of the output of $A \beta$ produced via initiation of translation from the AUG normally encoding Met 671 of $\beta$ APP.

One of the alternative mechanisms potentially capable of supporting the $\beta \mathrm{APP}$-independent $\mathrm{A} \beta$ production pathway is the initiation of transcription within the $\beta A P P$ gene. Another is the cleavage of $\beta$ APP mRNA at the appropriate position upstream of the Met671-encoding AUG. It is of interest to evaluate how much the alternative interpretations, positing that 5 -truncated $A \beta$-encoding mRNA is produced by a cleavage of the $\beta A P P$ mRNA or by the initiation of transcription within the $\beta$ APP gene, affect the $\beta$ APP mRNA amplification-based notions presented in the preceding sections. Surprisingly, very little. Indeed, consider the following. Both mechanisms under discussion are assumed to operate not constitutively but rather in an inducible manner in the disease. It follows that both need to be activated in AD. In both cases, the activation would require the production of an inducible protein.
In case of the initiation of transcription within the $\beta$ APP gene it would be a transcription factor or a co-factor; in case of a specific cleavage within $\beta$ APP mRNA it would be a distinct nuclease or its component. The production of both can be plausibly induced in the context of the integrated stress response, which is elicited in AD-affected cells via OMA1 - DELE1 - HRI axis of mitochondrial dysfunction. Thus, the timing and the mechanism of activation in both cases under discussion are the same as in the $\beta$ APP mRNA amplification scenario.

With regard to the operation of the alternative mechanisms and to the dynamics of the disease potentially caused by them, to show how little the alternative interpretations of the origin of 5 -truncated $A \beta$-encoding mRNA changes the argumentation offered in the $\beta$ APP mRNA amplification scenario, it is instructive to analyze three key figures presented above (Figures 7, 16 and 17) in the terms of alternative interpretations. The only part of Figure 7 that would be different is the box "RNA-dependent mRNA Amplification", which would be changed to " $\beta$ APP-independent generation of intraneuronally retained $A \beta$ ". The only component of Figure 16 that would be different under alternative interpretations is the box in the upper part of the "Engine" portion of the figure. Instead of "RNA-dependent $\beta$ APP mRNA amplification" it would stand for TF (transcription factor or a co-factor) required for the initiation of transcription within the $\beta$ APP gene, or for $\beta$ APP mRNA-cleaving enzyme, or simply for " $\beta$ APP-independent generation of intracellularly retained $A \beta$ " (by any means). Otherwise, the mode of operation would be identical to that shown in Figure 16: Intracellular accumulation of $A \beta$ produced in the $\beta$ APP proteolytic/secretory pathway ("Starter Motor") triggers mitochondrial dysfunction and, through its OMA1-DELE1-HRI axis, elicitation of the ISR and the activation, via induction of either a TF or a $\beta$ APP mRNA-cleaving enzyme or of any component required for operation of the $\beta A P P$-independent $A \beta$ production pathway, of the production of 5 '-truncated $A \beta$-encoding mRNA. The entire output of $A \beta$ derived from $\mathrm{N}$-terminal Met-containing C99 translated from 5'-truncated $\beta$ APP mRNA, or the bulk or at least a substantial part of it, is retained intraneuronally, further promoting mitochondrial distress and sustaining operation of the $\mathrm{AD}$ "Engine" driving the disease.

The OMA1-DELE1-HRI pathway is not the sole mediator of the $\mathrm{AD}$-associated, intraneuronal $\mathrm{A} \beta$-triggered elicitation of the ISR and consequent activation of the $\beta$ APP-independent $A \beta$ generation pathway. In Alzheimer's disease, $A \beta$ is known to activate, in a separate pathway, PKR, another eIF2 $\alpha$ kinase and 
an elicitor of the ISR. It appears, therefore, that in $\mathrm{AD}$, there is a redundancy in the $A \beta$-originated elicitation of the integrated stress response, presumably leading to the activation of the $\beta A P P-i n d e p e n d e n t ~ A \beta$ generation pathway in affected neurons. Moreover, the latter can potentially be initiated via intraneuronal $A \beta$-triggered processes other than the ISR. The generalized notion of the genesis of Alzheimer's disease and driving forces underlying its progression and endurance is depicted in Figure 18.
As for Figure 17, it would be literally identical in its entirety; the only difference would be the interpretation. Briefly, the interpretation of panels $\mathbf{A}$ and $\mathbf{B}$ would remain unchanged. In affected individuals, extracellular $A \beta$ produced in the $\beta$ APP proteolytic/secretory pathway starts accumulating early. This accumulation is accompanied by irreversible neurodegeneration. When neurodegeneration reaches a critical threshold, it manifests symptomatically. In this Paradigm, to achieve therapeutic effect,

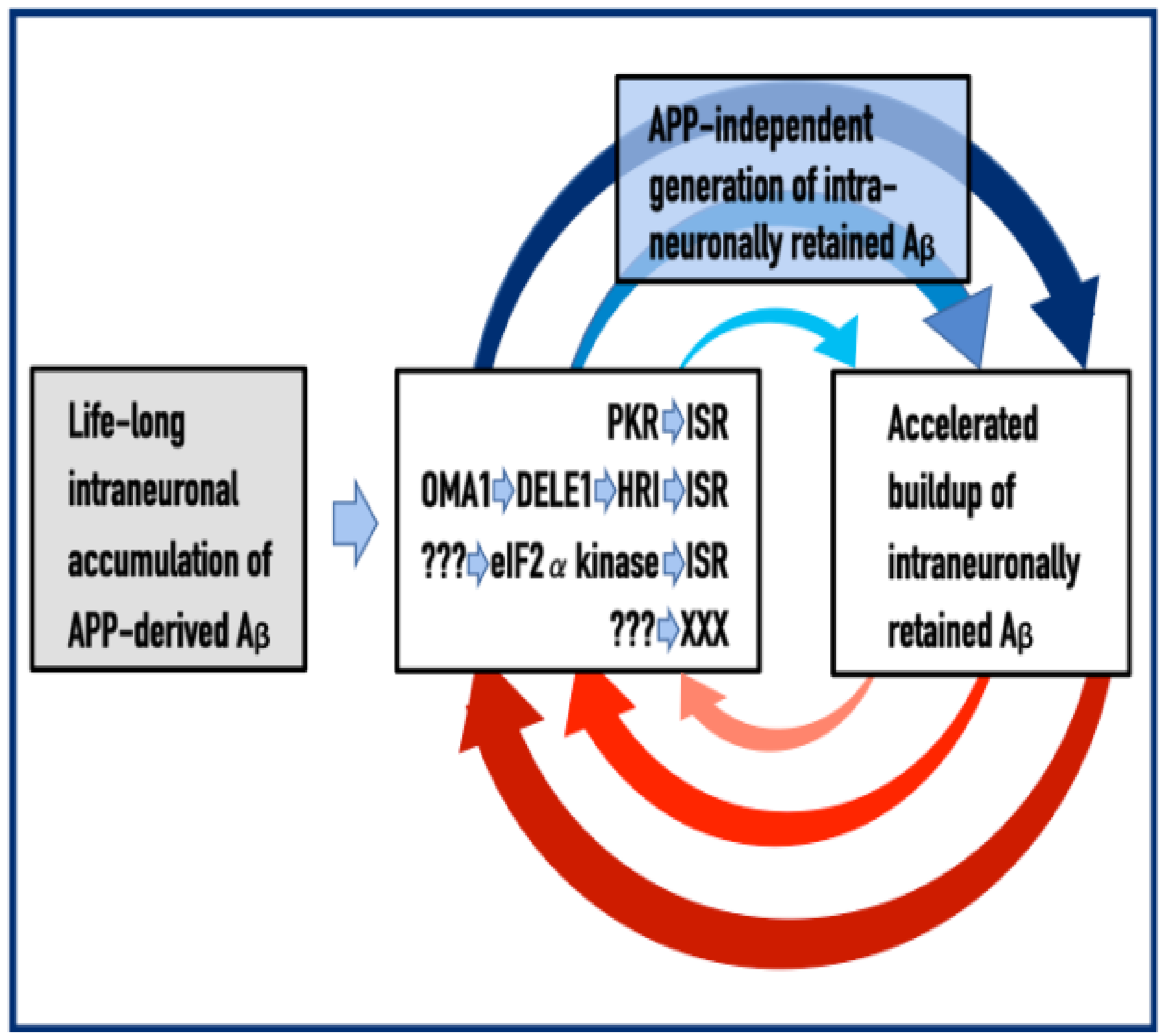

Figure 18: The etiology and Primum Mobile of Alzheimer's disease. Left Box (highlighted in grey): The "Starter Motor" - life-long intraneuronal accumulation of betaamyloid, through cellular uptake of secreted peptide and/or intracellular retention of a fraction of $\beta A P P$-derived A of the Figure). Middle Box: Several pathways, both actual (top two) and hypothetical (each line represent a pathway), of the A $\beta$-mediated elicitation of the integrated stress response or of the A $\beta$-triggered activation of yet unknown processes capable of initiating the $\beta A P P$ independent $A \beta$ production mechanism. Top Box (highlighted in blue): $\beta A P P$-independent generation of $A \beta$ via one of the following mechanisms - RNA-dependent $\beta A P P$ mRNA amplification; the internal initiation of transcription within the $\beta A P P$ gene; cleavage within $\beta A P P$ mRNA; the internal initiation of translation within $\beta A P P$ mRNA. Regardless of the mechanism employed, translation initiates at the AUG normally encoding Met671 of $\beta$ APP (which contiguously precedes Asp1 of C99 and of A 3 ) and results in C100 (N-terminal Met-containing $C 99$, converted post-translationally into C99 as described below), which is processed (by gamma-secretase cleavage) into $A \beta$ (or Met- $A \beta$ eventually converted to $A \beta$ ) that is retained intraneuronally. Right Box: Since the entire output, or at least the bulk, of A $\beta$ generated in the $\beta A P P$-independent pathway is retained within the cell, its intraneuronal levels rapidly increase. This sustains the operation of one or more of A $\beta$-mediated pathways shown in the Middle Box, which, in turn, support the

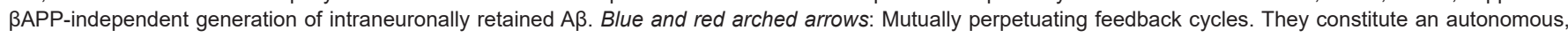
self-contained, two-stroke engine, the Engine that drives Alzheimer's disease. The disease commences and manifests symptomatically only following the activation of the AD Engine. Alzheimer's is, therefore, a "fast" disease that can be treated and potentially cured at the symptomatic stages. 
treatment should start early and continue for life. In panels $\mathbf{C}$ and $D$, illustrating the New AD Paradigm, intraneuronal accumulation of $\mathrm{A} \beta$ produced in the $\beta \mathrm{APP}$ proteolytic/secretory pathway does not cause any significant neurodegeneration. When a threshold T1 is reached, mitochondrial dysfunction is triggered, OMA1DELE1-HRI axis is activated and the integrated stress response is elicited. A transcription factor (or a co-factor) required for the initiation of transcription within the $\beta A P P$ gene is induced or the $\beta A P P$ mRNA-cleaving enzyme and/or a component of its complex are expressed under the ISR conditions. 5 '-truncated $A \beta$-encoding $\beta A P P$ mRNA is produced and translated into N-terminal Metcontaining $C 99$, which is processed into $A \beta$ (or Met- $A \beta$ ) that is retained intraneuronally. After a lag period when 5 '-truncated $\mathrm{A} \beta$-encoding RNA accrues and intracellular $\mathrm{A} \beta$ further accumulates, the persistent mitochondrial dysfunction, enabled by the operation of the $\mathrm{AD}$ "Engine" described above, causes a substantial neurodegeneration and symptomatic manifestation of the disease. In this AD Paradigm, therapeutic treatment can start and be effective any time prior to the activation of the TF or the $\beta A P P$ mRNA-cleaving machinery and even at the symptomatic stages. Panel $\mathbf{E}$ shows the kinetics of intracellular $A \beta$ accumulation in animals where suitably 5 '-truncated $A \beta$-encoding mRNA cannot be expressed or $\beta$ APP mRNA cannot be cleaved, or in humans where the operation of the AD "Engine" was successfully suppressed by therapeutic intervention. In either case, there is no significant neurodegeneration and no disease.

With regard to the exclusivity of $\mathrm{AD}$ to humans, in the framework of the alternative mechanisms it could be explained by nucleotide sequence specificity requirements for the internal initiation of transcription of the $\beta$ APP gene or for the $\beta$ APP mRNA cleavage, which are met in humans but not in animals. As described below, therapeutic strategies for Alzheimer's disease in the alternative mechanisms cases are also identical to those suggested in the $\beta$ APP mRNA amplification scenario.

Internal Initiation of Translation From The AUG Codon Normally Encoding Met671 Of BAPP Cannot Be Ruled Out In Alzheimer's Disease

Shortly after publication of the proposal that in Alzheimer's disease, $A \beta$ is generated independently of $\beta A P P$ via internal initiation of translation of $\beta$ APP mRNA from the AUG codon normally encoding Met671 of $\beta$ APP [5], several groups set out to test it. In one study [6], frameshift mutations were introduced upstream of the AUG in question. In another study, a stop codon was inserted upstream of it [7]. In both studies, the rationale was that if the internal initiation of translation from the AUG in question does occur, it would not be affected by the mutations upstream of the initiation site, and the Met671-initiated CTF of $\beta A P P$ would be generated from mutated $\beta$ APP mRNA. On the other hand, if the internal initiation of translation does not happen, no such CTF of $\beta$ APP would be produced from mutated mRNAs. In both studies the latter was shown to be correct. Accordingly, both studies concluded that the internal initiation of translation of $\beta$ APP mRNA from the AUG normally encoding Met671 of $\beta$ APP could be ruled out $[6,7]$.

But can it really be universally "ruled out"? The answer is a definitive "NO". This situation appears to be a typical case of comparing apples and oranges. The initial proposal [5] stipulated that in Alzheimer's disease, $A \beta$ can be produced via the internal initiation of translation from the auspiciously positioned AUG codon normally encoding Met671 of $\beta$ APP. The key words in the preceding sentence are "in Alzheimer's disease". It follows that this phenomenon is proposed to occur in an inducible manner, only in $\mathrm{AD}$-affected cells. The "ruling out" conclusion was reached, in both studies [6,7], with non-neuronal cells and certainly not under the $\mathrm{AD}$ conditions, As such, this conclusion may be correct for constitutive processes in normal cells but is simply inapplicable to inducible processes occurring in the disease (although, purely by chance, it could be correct in $\mathrm{AD}$ ). In terms of our discussion above, it could be envisioned that the integrated stress response triggered, via the OMA1-DELE1-HRI axis of mitochondrial dysfunction, by intraneuronal accumulation of $A \beta$ induces expression of a translation factor, or a co-factor that, in turn, enables internal initiation of translation of human $\beta$ APP mRNA from the AUG normally encoding Met671 of $\beta$ APP. In short, despite its initial rejection, this option is back on the table. It should be emphasized that all considerations of the preceding section equally apply to this option.

\section{C100 (N-Terminal Met-Containing C99) And N-Terminal Met-Containing $\mathbf{A} \beta$ Generated Independently Of $\beta$ APP Can Be Distinguished From Their Counterparts Produced In The BAPP Proteolytic Pathway}

As discussed above, in their proposal that translation of $\beta$ APP mRNA could be initiated from the AUG codon normally encoding Met671 of $\beta$ APP, Breimer and Denny suggested in 1987 [5] that the resulting translation product would be indistinguishable from the C99 fragment produced in the $\beta$ APP proteolytic pathway because the translation-initiating N-terminal Met would be cleaved-off co-translationally, as happens in the bulk of cellular proteins, by $\mathrm{N}$-terminal methionine aminopeptidases 1 and 2 (MAP1 and MAP2) [147]. Now, however, we know that this is not the case. For this cleavage to occur, both the translation-initiating $\mathrm{N}$-terminal Met and the penultimate residue have to fit in the active site of the enzyme. This is not always possible and depends on the size of a penultimate residue [148-150]. The co-translational cleavage of the N-terminal Met can occur only if it contiguously precedes one of the following seven amino acid residues [151] listed in order of increasing size (there are additional rules, which are not relevant to the case under discussion; some of them are invoked in cases discussed below): Glycine ( $\mathrm{Rg}=0$; $\mathrm{Rg}$ : radius of gyration of the side chain, in Angstroms), alanine ( $\mathrm{Rg}=0.77)$, serine $(\mathrm{Rg}=1.08)$, cysteine $(\mathrm{Rg}=1.22)$, threonine $(\mathrm{Rg}=1.24)$, proline $(\mathrm{Rg}=1.25)$ and valine $(\mathrm{Rg}=1.29)$. All other residues have larger $\mathrm{Rg}$ values culminating with arginine $(\mathrm{Rg}=2.38)$. With $\mathrm{Rg}$ of a penultimate residue larger than 1.29 Angstroms, the translation-initiating $\mathrm{N}$-terminal Met cannot be cleaved by MAP1 or MAP2. The Met671 of $\beta A P P$ is followed by the Asp672 ( $\mathrm{Rg}=1.43)$. It ensues that in the translation product initiated at the AUG normally encoding Met671 of $\beta$ APP, the $\mathrm{N}$-terminal Met is not removed co-translationally and the primary product is, in fact, C100 (i.e. N-terminal Met-containing C99) rather than C99.

If in a protein, the $\mathrm{N}$-terminal Met is not removed cotranslationally by MAP1 or MAP2, this does not mean that this protein would retain its translation-initiating Met for the entirety of its lifetime. In most such cases, $\mathrm{N}$-terminal Met is eventually 
removed by one of multiple aminopeptidases with a broad specificity [147]. A good example is mammalian cytoplasmic gamma-actin where the translation-initiating $\mathrm{N}$-terminal Met is followed by Glu $(\mathrm{Rg}=1.77)$ but is nevertheless efficiently removed post-translationally by an aminopeptidase other than MAP1/MAP2 [152]. Importantly, the removal of N-terminal Met by aminopeptidases other than MAP1 or MAP2 is always post-translational rather than co-translational. In such cases, the continuous translation of a protein (i.e. continuous input of N-terminal Met-containing polypeptides) would generate a certain steady-state population of protein molecules containing the $\mathrm{N}$-terminal Met despite its continuous post-translational removal. Such population could be detected by protein sequencing analysis.

If in Alzheimer's disease, translation initiating at the AUG normally encoding Met671 of $\beta$ APP indeed occurs (regardless the manner of generation of 5'-truncated $\beta A P P$ mRNA and including the possibility of internal initiation of translation), the $\mathrm{N}$-terminal Met would not be removed co-translationally for reasons discussed above but it would be removed post-translationally. We know this because neither $\mathrm{C} 100$, i.e. N-terminal Met-containing C99, nor $\mathrm{N}$-terminal Met-containing $\mathrm{A} \beta$ were seen in samples from $\mathrm{AD}$ affected brain [175]. This, of course, would be consistent with the lack of translation from the AUG encoding Met671 of $\beta$ APP in $\mathrm{AD}$, but if such translation does occur, $\mathrm{C} 100$ and Met- $\mathrm{A} \beta$ still would not be seen. This is because the analyzed samples were collected postmortem, and in dying cells protein synthesis stops while proteolytic processes remain active. In such circumstances, with no input of new N-terminal Met-containing molecules and with continuous removal of $\mathrm{N}$-terminal Met from pre-existing molecules, no N-terminal Met-containing C99 (C100) or A $\beta$ $($ Met-A $\beta$ ) would remain in a sample.

Provided that in $\mathrm{AD}$, translation initiates at the $\mathrm{AUG}$ normally encoding Met671 of $\beta$ APP from mRNA generated either in the $\beta A P P$ mRNA amplification pathway or by the initiation of transcription within the $\beta A P P$ gene, or by cleavage of $\beta A P P$ mRNA, or by the internal initiation of translation within $\beta A P P$ mRNA, the primary translation product would be $\mathrm{C} 100$ containing Met residue at its $\mathrm{N}$-end. The continuous generation of $\mathrm{C} 100$ and continuous post-translational removal of its $\mathrm{N}$-terminal Met, which converts it into C99, would generate a steady-state population of $\mathrm{C} 100$ of a size defined by the rate of its input versus the rate of the removal of its $\mathrm{N}$-terminal Met. The detection of such molecules would constitute indisputable proof of the occurrence of $\beta \mathrm{APP}$-independent generation of $\mathrm{C} 100 / \mathrm{C} 99$ and, consequently, of $A \beta$ (or Met- $A \beta$ ). If the rate of the removal of the $\mathrm{N}$-terminal Met from $\mathrm{C} 100$ is slower than the rate of $\mathrm{C} 100$ processing by gammasecretase, a population of intracellularly retained $\mathrm{N}$-terminal Metcontaining $A \beta$ would also be present, and could be detected, in $\mathrm{AD}$-affected cell or $\mathrm{AD}$ model system. If the opposite is true, i.e. the rate of removal of $\mathrm{N}$-terminal Met from $\mathrm{C} 100$ being faster than the rate of $\mathrm{C} 100$ cleavage by gamma-secretase, very little or no $\mathrm{N}$-terminal Met-containing $\mathrm{A} \beta$ peptides would be present in $\mathrm{AD}$ affected cells yet a steady-state population of $\mathrm{C} 100$ would persist. Therefore, the occurrence of C100 (N-terminal Met-containing C99) and, possibly, of N-terminal Met-containing $A \beta$ would identify the origin of these molecules and report the activity of the $\beta A P P-i n d e p e n d e n t A \beta$ production pathway. For reasons outlined above, the analysis of the presence of $\mathrm{C} 100$ or N-terminal Metcontaining $A \beta$ should be conducted in samples of living cells, i.e. samples from appropriate AD models. Human neuronal cell-based $\mathrm{AD}$ models suitable for such analysis are discussed below.

\section{Research Strategies In The New Alzheimer's Paradigm \\ Constructing AD Model: Activation of the Endogenous $\beta A P P-I n d e p e n d e n t A \beta$ Production Pathway in Human Neuronal Cells}

In designing research strategies for Alzheimer's disease, the major decision that needs to be made is the choice of the experimental model system. It can be argued that the best model system is human neuronal cells where processes could be easily activated that potentially lead to the endogenous $\beta$ APPindependent production of the C100 fragment of $\beta$ APP and, subsequently, of $A \beta$ or Met- $A \beta$, which can be detected. Approaches for the activation of the $\beta A P P$-independent $A \beta$ generation pathway and methods of detection of $\mathrm{C} 100$ and Met- $\mathrm{A} \beta$ produced in this pathway, as well as methodologies for evaluation of the nature of mechanisms involved, are described below. The main attribute of human neuronal cells as an $\mathrm{AD}$ model system is that it carries human $\beta A P P$ gene, with $\beta A P P$ mRNA eligible for the mRNA amplification process and/or for other processes resulting in the $\beta A P P-i n d e p e n d e n t ~ A \beta$ production possibly operating in human cells within the framework of the exclusivity of AD to humans. Sources of human neuronal cells are numerous and include neuronal cell lines, neuronal progenitor cell lines, and neuronal cells derived from iPSCs originated from either normal subjects or $\mathrm{AD}$ patients.

But even if such studies are successful and prove the occurrence of $\beta$ APP-independent generation of $A \beta$, a connection with Alzheimer's disease will have to be ascertained in order to establish the cause-and-effect relationship. In animal model systems, the evaluation of symptoms of the disease can provide such connection. Could "symptoms" of AD be observed in cultured neuronal cells? The answer is affirmative. Indeed, it was shown [153] that in cultured human neuronal cells, overproduction of $\mathrm{A} \beta$ can trigger formation of neurofibrillary tangles (NFTs), the major hallmark of the Alzheimer's disease; this study is analyzed in detail below. Observation of NFTs in human neuronal cells with the activated $\beta \mathrm{APP}$-independent $\mathrm{A} \beta$ production pathway would provide direct connection with the disease and be consistent with a notion that activation of $\beta$ APP-independent generation of intraneuronally retained $A \beta$ is required for the development and progression of $\mathrm{AD}$.

Methods for activation of $\beta$ APP-independent production of $A \beta$ from the endogenous $\beta$ APP gene in human neuronal cells are suggested by Figure 16 (as interpreted in the "Alternative Mechanisms" section above). One approach is to greatly accelerate the operation of the "Starter Motor", i.e. intracellular accumulation of $A \beta$ to levels sufficient to trigger mitochondrial dysfunction and to activate the self-perpetuating AD "Engine" - mutual feedback cycles of mitochondrial distress and $\beta$ APP-independent production of intraneuronally retained $A \beta$. This can be achieved by supplying cells with exogenous $A \beta 42$, either by importing the peptide or by expressing it from appropriate constructs. These constructs would encode $A \beta 42$, and translation would be initiated from the AUG codon preceding the $A \beta$ nucleotide sequence. However, for purposes of the proposed experiment, the translation-initiating AUG in a construct cannot precede the $A \beta$ sequence contiguously. 
This is because, as described below, a major detection assay for the activity of the $\beta$ APP-independent $A \beta$ production pathway is for the occurrence of $\mathrm{N}$-terminal Met-containing $\mathrm{A} \beta$ (along with C100). If, in a construct, the initiating AUG precedes the $A \beta$ coding sequence contiguously, the translation product would be Met- $A \beta$, and its presence would interfere with the detection of endogenously produced Met-A $\beta$.

Therefore, the design of a construct, or of a transgene, should be such that the initiating $\mathrm{N}$-terminal Met would be removed co-translationally, by MAP1 or MAP2. To achieve this, one of seven residues compatible with the co-translational removal of $\mathrm{N}$-terminal Met by MAP1/MAP2 [151] should be inserted between the initiating Met and the first aspartate of $A \beta$. The guide for which particular residue should be inserted is the $\mathrm{N}$-end rule, which defines the extent of stability (half-life) afforded to a polypeptide by its N-terminal residue [150]. According to this rule, the best choice would be valine. This, however, is not an option because, when the initiating Met is followed by Val or Thr (position 1, P1), the removal of the $\mathrm{N}$-terminal Met depends on the identity of a residue in P2. If it is Asp, Glu, or Pro, the N-terminal Met cannot be removed co-translationally [151]. Therefore, if in a construct/ transgene P1 is occupied by Val and P2 by Asp, the translation product would be Met-Val-A $\beta$. The second best choice, according to the $\mathrm{N}$-end rule, is Gly. If $\mathrm{P} 1$ is occupied by Gly, the $\mathrm{N}$-terminal Met would be readily removed co-translationally by MAP1/ MAP2 [151], and the resulting product would be Gly-A $\beta$, easily distinguishable from the anticipated Met-A $\beta$ generated in the endogenous $\beta$ APP-independent pathway.

Another approach to activate the endogenous $\beta$ APPindependent $A \beta$ production pathway in human neuronal cells is to bypass the stage of initial intracellular accumulation of $\beta$ APPderived $\mathrm{A} \beta$ and activate the main $\mathrm{AD}$ "Engine" directly (see Figure 16 , as interpreted in the "Alternative mechanisms" section above). The obvious target in this approach is to activate OMA1. The aim of such intervention is to trigger a cascade leading, through cleavage of DELE1 and export of one of its fragments to the cytosol [73,74], to activation of the HRI kinase [73,74,154], elicitation of the integrated stress response $[73,74]$, and the activation of the endogenous $\beta$ APP-independent $A \beta$ production pathway either via the RNA-dependent $\beta$ APP mRNA amplification pathway or through one of the alternative mechanisms described above.

In addition to the OMA1-DELE1-HRI-ISR axis, there is another OMA1-anchored axis: OMA1-OPA1, with OPA1 responsible for control of mitochondrial fragmentation and fusion [155-159]. There are, accordingly, two types of known OMA1 activators $[73,74]$, one that induces both OMA1-originating axes and that includes oligomycin and CCCP (mitochondrial ionofor Carbonyl Cyanide Chloro Phenyl hydrazine), and another type that activates only OMA1-DELE1-HRI axis. The latter includes doxycycline, antimycin A, and rotenone. Activation of only one of the OMA1originating axes, OMA1-DELE1-HRI, could be sufficient to activate the endogenous $\beta$ APP-independent $A \beta$ production pathway; if not, agents activating both axes simultaneously should be tried.

The third approach is to trigger phosphorylation of eIF $2 \alpha$ and elicitation of the integrated stress response independently of mitochondrial dysfunction, via stressor-specific activation of one of the other three kinases capable of eIF $2 \alpha$ phosphorylation, namely
PKR, GCN2, and PERK. This is a long-shot approach because it was shown that activated OMA1-cleaved DELE1 is capable of activating expression of certain transcription factors downstream of phosphorylated eIF2 $\alpha$ and independently of it $[73,74]$. DELE1activated gene expression may be needed to supplement the effects of the ISR in activating the $\beta$ APP-independent $A \beta$ production pathway; if this is the case, activation of the integrated stress response independently from mitochondrial distress would not be capable of triggering the $\beta A P P$-independent $A \beta$ generation pathway.

\section{Detection of the Activity of the $\beta A P P-I n d e p e n d e n t A \beta$ Production Pathway in Human Neuronal Cells}

As discussed above, the generation of $A \beta$ in the $\beta$ APPindependent manner in Alzheimer's disease can be executed by a variety of mechanisms. The common feature for all these mechanisms is that the primary translation product of the $\beta$ APPindependent $A \beta$ production pathway would in every case be the $\mathrm{C} 100$ fragment of $\beta$ APP retaining the translation-initiating methionine at its $\mathrm{N}$-terminus. This methionine will be eventually removed post-translationally by aminopeptidases with broad specificity, other than MAP1 and MAP2, and thus C100 would be converted into C99. Provided, however, that the input of C100 is continuous, its steady-state population would, despite the continuous removal of the N-terminal Met, persist in cells and could be detected. Moreover, if the rate of post-transcriptional removal of the translation-initiating $\mathrm{N}$-terminal methionine from $\mathrm{C} 100$ is slower, or at least not much faster, than the rate of its cleavage by gamma-secretase, a steady-state population of $\mathrm{N}$-terminal Met-containing $\mathrm{A} \beta$ molecules would, for reasons applied above, also occur in cells and could be detected by enrichment and sequencing. The detection of the C100 fragment of $\beta$ APP (N-terminal Met-containing C99), or of N-terminal Metcontaining $A \beta$, or of both would constitute irrefutable proof of the occurrence of the $\beta \mathrm{APP}$-independent $\mathrm{A} \beta$ generation pathway, and the detected molecules would function as both "identifiers" of their origin and reporters of the activity of the pathway.

Provided that the C100 fragment of $\beta$ APP or N-terminal Metcontaining $\mathrm{A} \beta$, or both species, are detected, it would be of interest to identify the mechanism(s) enacting the $\beta$ APP-independent $A \beta$ production. To ascertain or to exclude the involvement of RNA-dependent mRNA amplification in general and of the $\beta A P P$ mRNA amplification pathway in particular would be relatively straightforward. To test whether the RNA-dependent mRNA amplification process is operational in cells, reporters detailed in Figures 12 (Tier One) and 13 (Tier Two) above could be employed. Positive signals obtained with a reporter for Tier One or for Tier Two of the mRNA amplification pathway (or with both) would indicate that this pathway is operational. It would not prove, however, that $\beta A P P$ mRNA is being amplified. This can be evaluated by Next Generation Sequencing of cytoplasmic RNA followed by a search for sense/antisense $\beta$ APP RNA chimeric junction sequences of a type shown in Figure 15 above. The identification of such chimeric junction sequences would affirm the occurrence of $\beta$ APP mRNA amplification, and, in combination with the detection of C100 and/or N-terminal Met-containing $A \beta$ molecules, would leave little, if any, doubt that the latter are produced in the $\beta$ APP mRNA amplification pathway.

If such sense/antisense chimeric junction sequences were 
not detected, it would suggest that $\mathrm{C} 100$ and/or Met-A $\beta$ are produced by one of the alternative mechanisms described above. This conclusion could be confirmed by editing out of the $\beta$ APP genes segment corresponding to the TCE element of $\beta$ APP antisense RNA. The removal of this segment would prevent $\beta$ APP mRNA amplification but would not interfere with the alternative mechanisms possibly underlying the $\beta$ APP-independent $A \beta$ production pathway. Conceptually similar experiment could be performed by deleting a segment of the $\beta$ APP gene corresponding to the ICE element of $\beta$ APP antisense RNA. This, however, could interfere with the alternative mechanisms of $\beta$ APP-independent generation of $A \beta$. Another test could be assaying for the occurrence of a defined class of 5'truncated $\beta$ APP mRNA encoding CTF of $\beta$ APP where the first functional translation initiation codon would be the AUG normally encoding Met671 of $\beta$ APP. Since, as described above, the chimeric RNA end product of $\beta$ APP mRNA amplification would be undetectable by conventional means, the identification of such class of RNA would confirm the activity of one of the alternative mechanisms. Moreover, structure of this RNA could indicate the identity of an alternative mechanism of $\beta \mathrm{APP}$-independent generation of $\mathrm{A} \beta$, i.e. the presence of the 5 ' cap, for example, would suggest that the internal initiation of transcription of the $\beta \mathrm{APP}$ gene is in operation, whereas the lack of the cap structure would indicate $\beta$ APP mRNA cleavage, and the inability to detect a class of appropriately 5 '-truncated $\beta A P P$ mRNA would be consistent with the internal initiation of translation from the intact $\beta$ APP mRNA. Finally, with a proof for the operation of the $\beta A P P$-independent $A \beta$ production pathway obtained, a question would arise: Is this phenomenon related to Alzheimer's disease? To answer this question, one should look for the occurrence of neurofibrillary tangles, i.e. tauopathy, in human neuronal cells where the $\beta A P P-i n d e p e n d e n t ~ A \beta$ generation pathway has been activated. The rationale for this is presented in the following section.

\section{Tauopathy in Human Neuronal Cells with the Activated} $\beta A P P-I n d e p e n d e n t$ A $\beta$ Production Pathway: A Case Study

Below we present the analysis of a study where, for the first time, neurofibrillary tangles were observed in an Alzheimer's disease model system overexpressing $A \beta$. In our opinion, it is highly probable that in this study, involving human neuronal cells, investigators inadvertently activated endogenous $\beta$ APPindependent $A \beta$ production pathway. If this conclusion were correct, it would provide a connection with the major $\mathrm{AD}$ hallmark, suggest that formation of NFTs depends on activation of the $\beta A P P-i n d e p e n d e n t ~ A \beta$ production pathway and be consistent with the notion that this pathway is required for the development and progression of Alzheimer's disease.

In this study by Choi et al. [153], human $\beta$ APP harboring both, Swedish FAD mutation (K670N/M671L) and London FAD mutation (V717I), as well as presenilin 1 (PS1) with $\triangle \mathrm{E} 9$ FAD mutation, were overexpressed in human neural progenitor cells from polycistronic lentiviral constructs and differentiated in three-dimensional matrigel cultures. The effect of Swedish FAD mutation was discussed above. London FAD mutation facilitates generation of more toxic $\mathrm{A} \beta 42$ and so does PS1 with $\triangle \mathrm{E} 9 \mathrm{FAD}$ mutation. $\mathrm{AD}$ is characterized by two major hallmarks, $\mathrm{A} \beta$ plaques and neurofibrillary tangles. According to the amyloid cascade hypothesis of $\mathrm{AD}$, excessive accumulation of secreted
$\mathrm{A} \beta$ (manifested in the appearance of $\mathrm{A} \beta$ plaques) leads to formation of neurofibrillary tangles composed of aggregated hyperphosphorylated tau [160-162]. Prior to study under analysis [153], no AD model serially linked these two events. Mouse models of $\mathrm{AD}$ exhibit plaques deposition and $\mathrm{A} \beta$-induced synaptic and memory defects, but they do not recapitulate the NFTs pathology $[163,164]$. AD patient's iPSC-derived neurons show elevated levels of toxic $A \beta$ species and exhibit phosphorylated tau but form neither plaques nor neurofibrillary tangles [165-170]. In study by Choi et al. [153], the 3D-differentiated neuronal cells exhibited robust extracellular deposition of $A \beta$ plaques. They also exhibited high levels of detergent-resistant, silver-positive aggregates of phosphorylated tau (NFTs) both in the soma and neurites. Moreover, suppression of $A \beta$ generation in the $\beta A P P$ proteolytic pathway (by beta- or gamma-secretase inhibitors), starting early in differentiation, not only decreased extracellular deposition of $\mathrm{A} \beta$ but also attenuated tauopathy thus successfully recapitulating for the first time in a model system both $A \beta$ deposition and tau pathology. Authors of this study interpreted the results as serially linking the extracellular $A \beta$ deposition (pathological in Authors' view) to the formation of neurofibrillary tangles, and as validation of amyloid cascade hypothesis of $\mathrm{AD}$, which proposes that extracellular accumulation of $A \beta$ drives tauopathy.

The interpretation of the results of the study by Choi et al. [153] within the conceptual framework of the present article is quite different. Efficient expression from multiple lentiviral copies results in robust overproduction of mutated $\beta$ APP. As was discussed above, Swedish FAD mutation facilitates proteolytic cleavages of the $\beta$ APP mutant on intracellular membranes and, consequently, intraneuronal retention of the resulting $A \beta$ to much greater extent than seen with wild type $\beta$ APP [143]. Secreted A $\beta$ does not diffuse in matrigel and accumulates in the vicinity of the cell. A part of it aggregates and forms plaques. Another part of it is taken up by the cell and, because its concentration near the cell is high, the uptake is effective. Moreover, since most of secreted $A \beta$ is $A \beta 42$, its cellular uptake is efficient. This is because $A \beta 42$ is taken up preferentially, with the efficiency twice that of other $A \beta$ species [112]. These two processes, retention of a significant fraction of $\mathrm{A} \beta$ produced in the $\beta$ APP proteolytic pathway and cellular uptake of a portion of highly overproduced (and over-secreted) $A \beta$, result in rapid intracellular accumulation of $A \beta$ enriched, due to London FAD mutation, PS1 $\triangle \mathrm{E} 9$ FAD mutation, and differential preferential uptake of $A \beta 42$, in a more toxic $A \beta$ variant, $A \beta 42$. Significant intraneuronal accumulation of $A \beta 42$ produced in the $\beta$ APP proteolytic pathway alone could, possibly on its own, be sufficient to induce tauopathy. More likely, in our opinion, is the following.

Intraneuronal accumulation, by mechanisms described above, of toxic $A \beta 42$ produced in the $\beta$ APP proteolytic pathway reaches critical threshold and triggers mitochondrial dysfunction, activation of the OMA1-DELE1-HRI axis, phosphorylation of eIF $2 \alpha$ and elicitation of the integrated stress response. In the framework of the ISR, factor(s) necessary for the operation of the $\beta A P P-i n d e p e n d e n t A \beta$ generation pathway are expressed and the pathway is activated. The entire output of $\mathrm{A} \beta$ produced in this pathway, or at least the bulk or a substantial fraction of it, is not secreted but is retained intracellularly; this further dramatically increases intraneuronal levels of $A \beta$ and perpetuates, by sustaining mitochondrial dysfunction, activity of the $\beta$ APP-independent $A \beta$ 
production pathway. Sufficiently high levels of intraneuronal $A \beta$, resulting from the sustained, mitochondrial dysfunction-driven, operation of the $\beta \mathrm{APP}$-independent $\mathrm{A} \beta$ generation pathway, could be requisite for the tauopathy characteristic for Alzheimer's disease. Indeed, sufficiently increased levels of intracellular $A \beta$ could lead to the inhibition of the ubiquitin-proteosome system, facilitation of the build-up and phosphorylation of tau protein, and formation of NFTs [171-174]. If, in the system described by Choi et al. [153], $A \beta$ is produced independently from $\beta A P P$ via RNAdependent $\beta A P P$ mRNA amplification, this can occur only with endogenous $\beta$ APP mRNA and not with $\beta$ APP mRNA transcripts from lentiviral constructs. This is because during the assembly of lentiviral constructs, nucleotide sequence encoding $\beta$ APP mRNA segment corresponding to the TCE element of the antisense RNA has been removed, rendering lentiviral construct-derived $\beta A P P$ mRNA ineligible for the mRNA amplification process. On the other hand, if $\beta \mathrm{APP}$-independent $\mathrm{A} \beta$ generation occurs via one of the alternative mechanisms described above, it could operate with endogenous $\beta$ APP mRNA as well as with lentiviral constructsderived $\beta A P P$ mRNA transcripts.

In light of the above, there is high probability that in the experimental system described by Choi et al. [153], the $\beta$ APPindependent $\mathrm{A} \beta$ generation pathway has been inadvertently activated, either through RNA-dependent amplification of endogenous $\beta A P P$ mRNA or via an alternative mechanism. If this were indeed the case, steady-state populations of C100 (N-terminal Met-containing C99) and, possibly, N-terminal Metcontaining $A \beta$ would be present in cells and could be detected. We urge research groups working with this system to test for the presence of these molecules.

It should be noted that in the system under discussion [153], as, in fact, in human brain, $A \beta$ plaques are apparently not severely pathological, certainly not to the extent commonly assigned to them. Indeed, there is no good correlation between amount of plaques and $\mathrm{AD}$, and in a significant fraction of normal aged individuals, the amount of amyloid plaques in brain is similar to or greater than in a typical $\mathrm{AD}$ patient. It appears that plaques are mostly the consequence and reflection of $A \beta$ secretion, possibly even playing actually a protective role, and their amount and size are functions of the rate of $A \beta$ production in the $\beta A P P$ proteolytic/ secretory pathway. In this context, the observed "link" discussed above is not between $A \beta$ plaques and NFTs, as concluded by the Author's, but rather between $A \beta$ production, its intracellular levels and NFTs. Accordingly, in the study under discussion [153], betaand gamma-secretase inhibitors attenuated tauopathy not because they prevented plaque formation but because they suppressed, starting early in the progenitor's differentiation, the production of $\mathrm{A} \beta$ in the $\beta$ APP proteolytic pathway, and blocked its intracellular accumulation, i.e. stopped the AD "Starter Motor". Therefore, the results obtained by Choi et al. [153] could potentially be achieved in the absence of extracellular amyloid plaques, simply by overproduction, from a construct or a transgene, of $\mathrm{A} \beta 42$ (not requiring proteolytic processing) that would be retained intracellularly. In such a case, unless three-dimensional culturing is required for neural differentiation of progenitor, cells can be maintained in a liquid medium rather than in matrigel.

The great value of the study by Choi et al. [153] is the determination that tauopathy, the intraneuronal formation of neurofibrillary tangles and the major hallmark of Alzheimer's disease, can be achieved in human neuronal cell model of AD by overproduction of $A \beta$, thus establishing the connection between molecular processes under investigation and the disease.

\section{Therapeutic Strategies In The New AD Paradigm \\ Interfering with the AD "Starter Motor": Prevention of Intracellular Accumulation of $A \beta$ Produced in the $\beta A P P$ Proteolytic Pathway}

The preceding sections describe methods of generation of a potential human neuronal cells-based model of Alzheimer's disease. Such model, if and when available, would constitute a platform not only for studying pathogenic mechanisms of AD but also for drug screening. As with the development of approaches for activation of $\mathrm{AD}$-related processes, selection of therapeutic targets is also suggested and guided by Figure 16 (as interpreted in the "Alternative Mechanisms" section above). One obvious target is the $\mathrm{AD}$ "Starter Motor", i.e. intracellular accumulation to critical, mitochondrial dysfunction-inducing, levels of $A \beta$ produced in the $\beta$ APP proteolytic pathway. Its suppression would prevent the activation of the self-perpetuating AD "Engine" driving the disease. Several types of drugs that potentially can achieve this are available. It is sufficient to mention two types that were discussed in the Introduction section above. One type includes inhibitors of $\beta$ APP proteolysis such as verubecestat and other BACE inhibitors. These drugs reduce production of $A \beta$ in the $\beta$ APP proteolytic pathway, and therefore limit its intracellular retention and its secretion, which, in turn, also reduces its cellular uptake. In addition to $\beta$ APP, however, BACE has multiple cellular substrates and the feasibility of prolonged systemic administration of its inhibitors is questionable. Another type of potential drugs is $A \beta$ antibodies. These drugs reduce extracellular levels of $A \beta$ and, accordingly, reduce its cellular uptake. Because they do not interfere directly with intracellular retention of $\beta \mathrm{APP}$-derived $\mathrm{A} \beta$, their efficiency in reducing the rate of intracellular accumulation of $\mathrm{A} \beta$ is limited. The main problem, however, is that, to be effective, these types of drugs should be administered for very long periods of time, probably many years, prior to a typical timing of the manifestation of AD symptoms. For possible use of gamma-secretase modulators as potential $\mathrm{AD}$ drugs, please see below. Since these types of drugs do not affect operation of the main $\mathrm{AD}$ "Engine" (Figure 16, as interpreted in the "Alternative Mechanisms" section above), they would be completely inefficient once Alzheimer's disease symptoms manifested, indicating that the self-sustaining AD "Engine" has been switched on.

\section{Interruption of the AD "Engine": Blocking Mitochondrial Distress to the Integrated Stress Response Signaling Pathway}

Within the conceptual framework of the present article, the real "treatments" of Alzheimer's disease could only be those drugs that interfere with the operation of the self-perpetuating $\mathrm{AD}$ "Engine" that drives the disease. Such drugs, when developed, are expected to be effective in halting the disease in humans. Moreover, at the early symptomatic stages of $\mathrm{AD}$ in humans, when neurodegeneration is not yet substantial, it could be anticipated that they would reverse symptoms of the disease and thus provide the cure. Importantly, the same therapeutic strategies would be indicated regardless of the nature of a mechanism enacting $\beta$ APP- 
independent generation of $\mathrm{A} \beta$. Just as the $\mathrm{AD}$ "Engine" consists of two components, mitochondrial dysfunction and $\beta$ APPindependent production of intraneuronally retained $A \beta$, there are two potential categories of effective AD drugs. Each component of the $\mathrm{AD}$ "Engine" is anchored by a major physiological mechanism: The integrated stress response and the enzymatic machinery involved in $\beta$ APP-independent generation of $A \beta$. These types of mechanism could be vital for survival and cannot be interfered with therapeutically without significant deleterious consequences; they are "untouchable". There are, nevertheless, other targeting options and valid therapeutic approaches for both components of the AD "Engine".

One set of approaches includes those interfering with constituent elements of the OMA1-DELE1-HRI-ISR signaling pathway. Within this pathway, we cannot, as discussed above, target the ISR, including phosphorylation of eIF2a. It would, probably, be also futile to target the HRI kinase. This is because it was shown that when one of the four eIF2 $\alpha$ kinases is suppressed, another eIF2 $\alpha$ kinase can be activated, even by stressor(s) apparently specific for the suppressed kinase, and compensate for the inhibited kinase [27]; in other words, if the HRI is suppressed, stress of mitochondrial dysfunction is likely to result in elicitation of the integrated stress response through an eIF $2 \alpha$ kinase other than HRI. Moreover, as described above, it appears that cleaved DELE1 is capable of activating expression of various transcription factors downstream of phosphorylated eIF2 $\alpha$ independently of the latter $[73,74]$. This would make the inhibition of the binding of DELE1 fragment to HRI also inefficient as a therapeutic approach. This leaves two legitimate targets: OMA1 and DELE1. OMA1 anchors two axes: One leading, through DELE1 and HRI, to elicitation of the ISR, and another axis resulting in the activation of OPA1, a protein involved in regulation of mitochondrial fragmentation and fusion [155-159]. These two axes are distinct and independently regulated; as described above, OMA1 activators are known, which trigger either only OMA1 to ISR signaling pathway or both axes simultaneously $[73,74]$. In the same fashion, OMA1 inhibitors can be screened for, that suppress either only OMA1 to ISR pathway or both OMA1-anchored axes. When available, both types of inhibitors could be tested in human neuronal cells with activated $\beta A P P-i n d e p e n d e n t ~ A \beta$ production pathway for their ability to suppress this pathway. Inhibitors of DELE1 can also be of more than one type. They can suppress either DELE1 cleavage by OMA1 or the export of DELE1 fragment from mitochondria to the cytosol, or inhibit its activity downstream of eIF2 $\alpha$. On the other hand, inhibition of the interaction of DELE1 fragment with and activation of the HRI kinase would probably be futile for reasons given above. When available, DELE1 inhibitors should be tested in human neuronal cells-based $\mathrm{AD}$ model for their potential to interfere with $\beta A P P$-independent generation of $A \beta$. Provided that DELE1 and OMA1 inhibitors are similarly efficient in suppression of the $\beta A P P$-independent $A \beta$ production pathway, it would be preferable to target DELE1 because interfering with OMA1 could potentially be significantly more deleterious in $\mathrm{AD}$ patients.

\section{Interruption of the AD "Engine": Preventing Intracellular Accumulation of $A \beta$ Generated in the $\beta$ APP-Independent Pathway}

The second category of potential $\mathrm{AD}$ drugs that interfere with operation of the $\mathrm{AD}$ "Engine" includes agents that prevent intraneuronal accumulation of $A \beta$ produced in the $\beta$ APP-independent pathway. The primary translation product of this pathway is the C100 fragment of $\beta$ APP ( $\mathrm{N}$-terminal Met-containing C99, eventually converted post-translationally into $\mathrm{C} 99$, as described above). The production of $A \beta$ (or $N$-terminal Met-containing $A \beta$ ) requires cleavage of this fragment by gamma-secretase. When C100 is produced in non-neuronal cells from a construct not encoding a secretion signal peptide, it is, nevertheless, cleaved on plasma membrane and the resulting $A \beta$ is secreted [138]. This occurs because C100/C99 contains an internal trans-membrane domain [138]. As stated above, the two core notions of the New Paradigm of $\mathrm{AD}$ are: $\mathbf{1}$. In the disease, in addition to the $\beta \mathrm{APP}$ proteolytic process, $\mathrm{C} 100$ and, subsequently, $A \beta$ are produced independently of $\beta$ APP and 2. The entire output of A $\beta$ generated in the $\beta$ APPindependent pathway, or the bulk or at least a substantial fraction of it, is retained intraneuronally. To satisfy the latter, it has to be presumed that, to escape secretion of $\mathrm{A} \beta, \mathrm{C} 100$ (or C100-derived C99) is cleaved by gamma-secretase on an internal cellular membrane. Such type of cleavage indeed occurs in neuronal cells [130], and it can be assumed that $\mathrm{C} 100$ produced in the $\beta$ APPindependent pathway is processed into $A \beta$ in such manner. Therefore, an agent could be screened for, which facilitates a shift of the location of gamma-secretase cleavage of C100 (or C99; both, C100- and $\beta$ APP-derived) from internal membranes to the plasma membrane, and, consequently, expedites secretion of the resulting $A \beta$. This is plausibly achievable because the desired shift would occur from one physiological condition to another. Such agent, if found, could constitute an efficient AD drug. This is because within the framework of the New Paradigm of AD, the $A \beta$ toxicity is attributed primarily to the intraneuronally retained peptide, with extracellular $A \beta$, which is continuously cleared out, of no great concern. Moreover, if excessive extracellular A $\beta$ does cause problems, these problems can be addressed by simultaneous administration of existing $\mathrm{A} \beta$ antibodies drugs. If such agent is found, it should be tested for its ability to stop, by collapsing intraneuronal $\mathrm{A} \beta$ levels, the $\mathrm{AD}$ "Engine" and, consequently, to inhibit $\beta$ APP-independent production of $A \beta$.

Another approach to reduce or prevent intraneuronal accumulation of $\mathrm{A} \beta$ produced in the $\beta \mathrm{APP}$-independent pathway is to interfere with its generation. It could be argued that this can be done by inhibition of gamma-secretase and/or activation of alpha-secretase. This is, however, apparently not the case. Gammasecretase is a prominent component of the Notch signaling pathway. It has numerous cellular substrates other than C99 and its inhibition by candidate $\mathrm{AD}$ drugs was shown to be lethal in human clinical trials. Alpha-secretase, a member of the ADAM family of proteases, also has well over hundred known cellular substrates. Its activators were, in fact, developed but did not fair well in human clinical trials. A class of promising agents that could possibly be of therapeutic value is modulators of gamma-secretase [176-183]. Cleavage of C99 by gamma-secretase is not a single-step process. The initial cleavage is made downstream of the future $\mathrm{C}$-terminus of $A \beta$, between residues 49 and 50 of $A \beta$ (dominant site in wild-type cells) or between residues 48 and 49 of $A \beta$. Subsequent processive cleavages, proceeding every few amino acids in the upstream direction, result in the final gamma-cleavage and release of $A \beta$. The final gamma-cleavage can occur at various positions starting with residue 43 of $A \beta$ (generating $A \beta 43$ ) and down to position 37 of $A \beta$ (generating $\mathrm{A} \beta 337$ ). Typically, gamma-secretase processing of C99 results in a spectrum of $A \beta$ species in neuronal cells, with a peak at 
certain position (e.g. A $\beta 40$ in wild- type neuronal cells and $A \beta 42$ in some FAD mutants). "Modulators" of gamma-secretase are agents that facilitate generation of shorter, less toxic, $A \beta$ species. Under a gamma-secretase modulator, a spectrum remains but the peak is shifted upstream ("inverse modulators" also have been found that facilitate generation of longer $A \beta$ variants, i.e. a shift of the peak of a spectrum of $\mathrm{A} \beta$ species in the opposite direction). Potentially, gamma-secretase modulators could be effective as $\mathrm{AD}$ drugs. Their effectiveness, however, would be limited since intracellular levels of $A \beta$ would not be reduced, and toxic $A \beta$ species would still be produced, even in the modulator-shifted spectrum. In fact, they could be more effective in prevention of the disease, at the "Starter Motor" activation stage, if their administration is initiated early enough.

\section{Interruption of the AD "Engine": Activation (Yes Activation!) of Beta-Secretase as Highly Promising Therapeutic Strategy}

It would be hard to find an example that illustrates drastic difference between the Old and the New Paradigms of Alzheimer's disease better than one described below. The best potential AD drug, developed within confines of the Old AD Paradigm (in which $A \beta$ is produced, in health and disease, solely in the $\beta A P P$ proteolytic/secretory pathway), is a BACE inhibitor, highly efficient in animal models but completely ineffective in mitigating human $\mathrm{AD}$ symptoms. As argued below, potentially, the best $\mathrm{AD}$ drug effective in human patients and designed within the framework of the New AD Paradigm (i.e. in AD, in addition to the $\beta$ APP proteolytic/secretory pathway, $A \beta$ is also generated in the $\beta$ APP-independent pathway and retained intraneuronally) could be an agent with the diametrically opposite activity, namely a BACE activator.

If one envisions the ideal way to reduce or suppress generation of $A \beta$ in both the $\beta A P P$ proteolytic pathway and, importantly, in the $\beta$ APP-independent pathway, it would be to introduce an activity that cleaves within $\mathrm{A} \beta$ portion of $\mathrm{C} 100$ or $\mathrm{C} 99$ and, possibly, even within $\mathrm{A} \beta$ as long as it remains within the cell. The resulting fragments would be harmless, non-toxic or non-amyloidogenic. In other words, such cleavage would be amyloidolytic. As in a dream-come-true scenario, such activity is already present in $\mathrm{A} \beta$-producing cells, both normal and $\mathrm{AD}$-affected. Moreover, this activity is a familiar one: BACE1, i.e. beta-secretase. Indeed, consider the following [184-187]. 1. BACE1 was shown to cleave not only (albeit predominantly) at the $\beta$-site, prior to residue 1 of $\mathrm{A} \beta$ (between residues 671 and 672 of $\beta$ APP) but also at the $\beta$ '-site between residues 10 and 11 of $A \beta$. Whereas cleavage at the $\beta$-site generates C99 and, after gamma-secretase cleavage, A $\beta 1$-XX (XX stands for any number between 37 and 43 ), cleavage at the $\beta$ '-site generates C89 and, after gamma-secretase cleavage, A $\beta 11-X X .2$. BACE1 cleaves with equal efficiency at the $\beta$ '-site of both $\beta$ APP and C99. 3. There is every reason to believe that intracellular $A \beta$ would also be a substrate for and target of the $\beta^{\prime}$-cleaving activity of BACE1 (this wasn't tested on presumption that cleavage of C99 by gamma-secretase is synonymous with secretion of $A \beta$, and, therefore, its unavailability to cellular enzymes). 4. Overproduction of human BACE1 in mice increases both the rate of cleavage at the $\beta$ '-site and the ratio of $A \beta 11-X X / A \beta 1-X X$. 5. Overproduction of human BACE1 strongly decreases deposition of extracellular $A \beta$ in mouse brain. 6. Protective effect of the Icelandic FAD mutation $\mathrm{A} 673 \mathrm{~T}$ is associated with and appears to be due to the increased rate of BACE1-mediated cleavage at the $\beta^{\prime}$-site. Since C100 (eventually converted to C99) generated in the $\beta$ APP-independent $A \beta$ production pathway, and intracellularly retained $A \beta$ (or Met$A \beta$ ) produced from it, are valid substrates for BACE1 cleavage at the $\beta^{\prime}$-site, the increase of the activity of BACE1, or its small molecule-mediated differential shift toward preferential $\beta$ '-site cleavage, or both, could be of significant therapeutic value in AD.

Moreover, recent studies showed that BACE1 is also capable of cleavage between residues 34 and 35 of human $A \beta$ (yet unnamed BACE1 cleavage site), and that the rate of this cleavage correlates with the activity of BACE1 and substantially increases when BACE1 is overexpressed; this cleavage generates an intermediate $(\mathrm{A} \beta 34)$ in $\mathrm{A} \beta$ degradation/clearing process [188-192]. Taken together, the above observations strongly suggest that a sufficient increase in the activity of BACE1 in Alzheimer's disease, or its differential shift toward cleavages at the $\beta$ ' and/or $34 / 35$ sites of $A \beta$, could initiate amiloidolytic processing of C100/C99 and A $\beta /$ Met$A \beta$, reduce intracellular $A \beta$ to harmless form, and be of substantial therapeutic benefit. There is, therefore, good probability that an agent capable of sufficient activation of beta-secretase would constitute an efficient AD drug. Any increase in BACE1 activity could be useful but the ideal agent to be screened for would be the one that differentially increases BACE activity at $\beta^{\prime}$ and A $334 / 35$ sites versus its activity at the $\beta$-site. When available, such agent could be tested for its ability to suppress production of $A \beta$ in the $\beta A P P-i n d e p e n d e n t ~ A \beta$ generation pathway, and thus to stop the AD "Engine" in human neuronal cells-based AD model. The resulting drug is expected to be effective in halting the disease in humans and even reversing and curing it at the early symptomatic stages of $\mathrm{AD}$.

\section{Therapeutic Strategies for Symptomatic AD: General Conclusions}

In light of the above discussion, it can be concluded that Alzheimer's disease can be treated, even when its symptoms have already manifested, and, moreover, potentially can be cured at the early symptomatic stages. Of two phases associated with the disease, the "Starter Motor" and the AD "Engine", only the latter can be productively targeted therapeutically. The initial manifestation of symptoms of the disease signify its beginning rather than the endstage, and coincide causatively with the activation of the AD "Engine", which, in its operational state, is completely autonomous and fully independent of the "Starter Motor" (just as a car's engine is when it is switched on). Therefore, targeting the "Starter Motor" at the symptomatic stages, for example by inhibiting BACE1, would be futile.

As for the AD "Engine", there are two conceptual approaches of targeting it. One is to interfere with the elicitation of the integrated stress response, and, consequently, with the activation of the RNAdependent mRNA amplification pathway or an alternative process capable of generating A $\beta$ independently of $\beta$ APP. This approach could be effective but is potentially problematic because of the redundancy in the elicitation of the ISR and the possibility that in AD, $\beta$ APPindependent production of $A \beta$ could be initiated by intraneuronal $\mathrm{A} \beta$-triggered processes other than the ISR, as shown in Figure 18. Indeed, the inhibition of the OMA1-DELE1-HRI signaling pathway would not affect the capacity of $A \beta$-activated PKR kinase to activate and sustain the ISR. Supplementary suppression of PKR, on the other hand, may trigger compensatory activation of other eIF2 $\alpha$ kinase(s) that would sustain the operation of the ISR and of a mechanism 
generating $A \beta$ independently from $\beta$ APP. Better understanding of dynamics of the interaction of A $\beta$-OMA1-DELE1-HRI-ISR and A $\beta$ PKR-ISR pathways in Alzheimer's disease would be helpful in defining proper targets in this approach.

Another, apparently the preferable, approach to interfere with the $\mathrm{AD}$ "Engine" is to reduce the intraneuronal amounts of $\mathrm{A} \beta$ to the levels that are not sufficient to support operation of the pathways leading to the activation of the integrated stress response or to the ISR-unrelated initiation of $\beta$ APP-independent production of $A \beta$. This would preclude the activity of a mechanism generating $A \beta$ independently of $\beta A P P$, and the AD "Engine" would collapse. There is more than one way to enact this strategy. One is to export $A \beta$ out of the cell. This can be achieved by shifting the predominant site of gamma-cleavage from internal membranes to plasma membranes (both are physiologically utilized sites) and, consequently, facilitating the secretion of the resulting $A \beta$. Another way is to facilitate the amyloidolytic processing of $\mathrm{C} 99$ and $\mathrm{A} \beta$ by activating BACE1 and/or its activities capable of cleaving within $A \beta$ (or Met- $A \beta$ ) or $A \beta$ segment of $C 99$ (or C100). Moreover, there is a good probability that BACE1 activators would stimulate BACE2, which is also capable of cleaving in the middle of $A \beta$, or at least facilitate the identification of BACE2 activators. $A \beta$ cleaving activity of BACE2 would not only supplement that of BACE1 but would also complement it because of their distinctly different cellular localization. Both strategies hold great promises of producing a drug capable of treating and, potentially, curing Alzheimer disease at the symptomatic stages.

\section{Conclusion}

The present article describes the New Paradigm of Alzheimer's disease. In the Old Paradigm, formalized in the Amyloid Cascade Hypothesis $(\mathrm{ACH})$ theory of $\mathrm{AD}$, beta amyloid is produced solely in the $\beta$ APP proteolytic/secretory pathway. The overproduction of $A \beta$ results in its extracellular accumulation commencing early in life. This triggers a cascade of molecular and cellular events, including formation of neurofibrillary tangles, which results in neurodegeneration. When the extent of neurodegeneration reaches critical levels, symptoms of the disease are manifested. In this Paradigm, Alzheimer's disorder is a quintessential "slow" disease. The $\mathrm{ACH}$ clearly defined therapeutic targets, which included key events of $\beta$ APP proteolysis, as well as secreted extracellular $\mathrm{A} \beta$. Eventually, a number of candidate $\mathrm{AD}$ drugs, highly effective in animal model systems, was developed. Of those, especially successful were inhibitors of beta-sectretase that not only prevented the emergence of $\mathrm{AD}$ symptoms, but also reversed them when administered after symptomatic manifestation of the disease in animal model systems. At this point, there was every reason to believe that a solution to the Alzheimer's disease problem is at hand. This, however, proved not to be the case.

Both the Old and the New Paradigms share the following common point of departure: The overproduction of betaamyloid is the causative basis of Alzheimer's disease. The rest of the notions of the New Paradigm are distinctly different from those of the Old one. Formulation of the New Paradigm theory of Alzheimer's disease was necessitated by close analysis of the results of massive human clinical trials of candidate Alzheimer's disease drugs that performed outstandingly in animal studies. All tested candidate AD drugs, however, failed in human clinical trials as spectacularly as they succeeded in animal studies. Or did they? Whereas they indeed showed no efficacy whatsoever, none of symptoms mitigation or clinical improvement in $\mathrm{AD}$ patients, they nevertheless performed perfectly within confines of their design and purpose. For example, a BACE inhibitor verubecestat penetrated the brain of $\mathrm{AD}$ patients, greatly inhibited $\beta \mathrm{APP}$ cleavage, and strongly suppressed extracellular levels of $A \beta$. It did all this with about the same efficiency it showed in animal studies where it, indisputably, succeeded in mitigating symptoms of the disease. Why did it fail to do so in human clinical trials?

This failure, apparently inexplicable within the confines of the $\mathrm{ACH}$, seems, therefore, as good an occasion as any to apply the central dictum of Sherlock Holmes: “... when you eliminated the impossible, whatever remains, however improbable, must be the truth" [192]. In the case under discussion, after the elimination of the "impossible", including elements of the $\mathrm{ACH}$, and provided that Alzheimer's disease is indeed caused by the overproduction of $A \beta$, a notion that is strongly supported by experimental data, the "however improbable" that is consistent with the outcomes of human clinical trials of AD candidate drugs is the following. 1. In addition to the $\beta$ APP proteolytic/secretory process, in Alzheimer's disease in humans $A \beta$ is also produced in the $\beta A P P$-independent pathway. This pathway is active only in $\mathrm{AD}$ patients. It does not operate in animals and healthy humans. 2 . The entire output, or the bulk, or at least a substantial fraction of the output of the $\beta$ APPindependent $A \beta$ generation pathway is retained intraneuronally, and it is this pool of intraneuronal beta-amyloid that causes Alzheimer's disease.

The "improbable" notions described above constitute the core of the New Paradigm of AD. According to it, the Alzheimer's disease starts with the activation of the $\beta$ APP-independent $A \beta$ production pathway. $A \beta$ generated in this pathway is retained intraneuronally and, when its sufficient levels are reached, initiates and maintains a cascade of molecular and cellular events, including formation of NFTs, that results in symptomatic manifestation of the disease and, ultimately, in neuronal death. In this Paradigm, Alzheimer's disorder is a "fast" disease that can be treated at the symptomatic stages. Within the conceptual framework of the New Paradigm of $\mathrm{AD}$, the mechanism generating beta-amyloid independently of $\beta$ APP constitutes the essence of Alzheimer's disorder, and its elucidation is crucial for the understanding and treatment of the disease.

Several cellular mechanisms are capable of $\beta$ APP-independent generation of $A \beta$. All of them share the following multiple common features. (a) The nature of the activation of the $\beta$ APPindependent $A \beta$ production pathway is conceptually identical for every potential mechanism and entails the ISR-mediated induction of its crucial component(s). (b) In every potential mechanism of $\beta A P P-i n d e p e n d e n t A \beta$ generation, translation initiates at the AUG codon normally encoding Met671 of $\beta$ APP and results in C100, i.e. $\mathrm{N}$-terminal Met-containing C99, which is subsequently cleaved by gamma-secretase to produce $A \beta$ (or Met- $A \beta$ ). (c) In every potential mechanism, N-terminal Met of C100 is removed post- rather than co-translationally. (d) In every potential mechanism of $\beta$ APPindependent $A \beta$ generation, the entire $A \beta$ (or Met- $A \beta$ ) output, or the bulk, or a significant portion of it, is retained intraneuronally. (e) Once activated, every potential $\beta$ APP-independent mechanism sustains, through generation of intraneuronally retained $A \beta$, the activity of the self-perpetuating "Engine" that drives Alzheimer's disease. 
In every potential mechanism of $\beta$ APP-independent generation of $A \beta$, expression of a crucial component(s) that enables its operation is induced by the integrated stress response elicited by activation of the HRI kinase that occurs in the context of the OMA1-DELE1-HRI signaling pathway. This signaling pathway is activated by mitochondrial dysfunction, which, in turn, is triggered by a life-long intraneuronal accumulation to critical threshold levels of $\mathrm{A} \beta$ produced in the $\beta \mathrm{APP}$ proteolytic/secretory pathway. There are two sources of such intracellular $\beta$ APP-derived A $\beta$. One is cellular uptake of secreted $A \beta$. This mechanism is facilitated by ApoE4, a major genetic risk factor of AD. Secreted $A \beta$ species are taken up differentially: $A \beta 42$, the most "toxic", i.e. amyloidogenic, of beta-amyloid species, is taken up twice as efficiently as any other $A \beta$ variant. Another source is the retention within the cell of a small fraction of $\beta$ APP-derived A $\beta$ generated by gamma-secretase cleavage of $\mathrm{C} 99$ at intracellular rather than at plasma membranes. This can occur at various locations of intracellular $A \beta$ generation, some of which are limited to neurons. Therefore, intracellular retention of $\beta \mathrm{APP}$-derived $\mathrm{A} \beta$ is more likely to occur in neurons than in non-neuronal cells. Life-long intraneuronal accumulation of $\beta$ APP-derived $A \beta$ reaches critical threshold levels and triggers mitochondrial distress, thus activating mitochondrial OMA1 protease, which cleaves mitochondrial protein DELE1. One of the resulting DELE1 fragments is exported from mitochondria to the cytosol where it binds to and activates HRI kinase. In turn, HRI phosphorylates eIF $2 \alpha$, thus eliciting the integrated stress response. The ISR suppresses global cellular protein synthesis but activates expression of selected transcription factors, some of which act as inducible components (or enable expression of those components) of a mechanism underlying the $\beta \mathrm{APP}$-independent $\mathrm{A} \beta$ production pathway, which is then activated. With the entire output (or the bulk or at least a substantial fraction of the output) of this pathway retained intracellularly, increased levels of intraneuronal $A \beta$ promote further mitochondrial dysfunction, the OMA1 to DELE1 to HRI signaling pathway is maintained, and the integrated stress response and, consequently, the $\beta \mathrm{APP}$-independent $\mathrm{A} \beta$ production pathway, are sustained, thus generating self-perpetuating $\beta$ APPindependent $A \beta$ generation/mitochondrial dysfunction mutual feedback cycles. In the described sequence of events, therefore, accumulation of $\beta A P P$-derived $A \beta$ to levels sufficient to trigger mitochondrial dysfunction plays the role of a car's starter motor in getting car's engine moving in a self-sustainable manner. In human neurons, once "Starter Motor" is switched on, it activates the "Engine" that drives Alzheimer's disease: Self-propagating mutual feedback cycles of mitochondrial distress-mediated $\beta$ APPindependent generation of intraneuronally retained $A \beta$, which, in turn, promotes mitochondrial dysfunction.

At least four distinct mechanisms are capable of generating $\mathrm{A} \beta$ in the $\beta \mathrm{APP}$-independent manner. One of them is mammalian RNA-dependent mRNA amplification, a mechanism that was implicated in overproduction of specific proteins, such as both chains of globin and all three chains of laminin, in mammalian cells. This process operates via self-priming of the antisense RNA and the extension of its 3'-terminus into sense-oriented molecule. This results in a hairpin-structured sense/antisense chimeric intermediate. Subsequent strand separation and cleavage at the single-stranded loop of a hairpin structure produces chimeric RNA end product, an mRNA molecule containing antisense portion at its 5' end. If self-priming of antisense RNA strand occurs within its segment corresponding to the 5' UTR of a conventional mRNA, the chimeric RNA end product of amplification would retain the complete coding capacity of its conventional mRNA progenitor. If, however, self-priming structure is assembled within a segment of antisense RNA corresponding to the coding region of an mRNA, the RNA end product of amplification would contain only 3' portion of the coding region of conventional mRNA progenitor and could encode only a CTF of the conventional polypeptide. If $\beta A P P$ mRNA amplification were responsible for $\beta A P P-i n d e p e n d e n t$ generation of $A \beta$, the latter scenario would occur. Indeed, it was shown that $\beta$ APP antisense strand is capable of self-priming within its segment corresponding to the coding region, and, therefore, of mediating asymmetric $\beta A P P$ mRNA amplification resulting in mRNA encoding only CTF of amyloid precursor protein. The first AUG codon in such mRNA is the one encoding Met671 of $\beta$ APP; its translation, therefore, would result in the $\mathrm{C} 100$ ( $\mathrm{N}$-terminal Met-containing C99) fragment of $\beta A P P$, and would eventually produce $A \beta$ (or Met- $A \beta$ ) in the $\beta A P P-i n d e p e n d e n t$ manner. The activation of the $\beta$ APP mRNA amplification pathway would require ISR-induced expression of, at least, a processivity co-factor of RdRp and, possibly, other inducible components of the RdRp complex.

Another mechanism capable of $\beta$ APP-independent generation of $A \beta$ in Alzheimer's disease is the internal initiation of transcription within the coding region of the $\beta A P P$ gene. The mRNA product of such mechanism would be conceptually identical to the RNA end product of $\beta$ APP mRNA amplification, namely an mRNA encoding only C-terminal fragment of amyloid precursor protein, where the first functional translation initiation codon is the AUG normally encoding Met671 of $\beta$ APP. The activation of the internal initiation of transcription within the coding region of the $\beta$ APP gene would require induction of a suitable transcription factor (or a co-factor) under conditions of the integrated stress response.

The third mechanism potentially responsible for $\beta$ APPindependent generation of beta-amyloid in Alzheimer's disease is the cleavage of the intact $\beta A P P$ mRNA in a suitable position within its coding region. One of the two resulting fragments of such process would be of exactly the same type as RNA products of two potential $\beta \mathrm{APP}$-independent $\mathrm{A} \beta$ generation mechanisms described above, namely an mRNA encoding only a C-terminal fragment of $\beta$ APP, where the first translation initiation codon is the AUG normally encoding Met671 of $\beta$ APP. Activation of the $\beta A P P$ mRNA cleavage within its coding region would require integrated stress response-enabled expression of an enzyme (or an enzyme's component) capable of such cleavage. The resulting mRNA of interest would be cap-less. This, however, would not be a handicap since under the ISR conditions, translation of cap-less mRNAs occurs preferentially.

The forth potential mechanism of production of beta-amyloid in the $\beta A P P$-independent manner is the internal initiation of translation within the coding region of $\beta A P P$ mRNA, at the AUG normally encoding Met671 of $\beta$ APP. It would produce, independently of $\beta$ APP, the same type of protein product as the candidate mechanisms discussed above: C100 (N-terminal Metcontaining C99 fragment of $\beta \mathrm{APP}$ ) that would eventually be processed into $A \beta$ (or Met- $A \beta$ ). In fact, such mechanism initiating at the AUG normally encoding Met 671 of $\beta$ APP, was proposed early on, soon after identification of the human $\beta$ APP gene, but 
was eventually "ruled out". However, for the reasons articulated in the present article, this option should be placed back on the table. The activation of the internal initiation of translation would require the integrated stress response-induced expression of a translation factor or of its co-factor.

Thus, all four potential mechanisms of $\beta$ APP-independent generation of $\mathrm{A} \beta$ would produce, as their primary translation products, identical CTFs of $\beta$ APP. This is because, in every case, translation would initiate from the same AUG, normally encoding Met671 of $\beta$ APP. Not only does this AUG codon contiguously precede the nucleotide sequence encoding $A \beta$, it is also situated within an optimal translation initiation context. Such auspicious positioning of this particular AUG codon is, in fact, a rarity. Please consider: Of all twenty AUG codons encoding methionine residues in human $\beta$ APP, only the AUG in question, not even the AUG encoding Met which initiates translation of $\beta A P P$, is situated within an optimal translation initiation context. Accordingly, the primary translation product, identical in every potential mechanism of $\beta$ APP-independent production of $A \beta$, would be the $\mathrm{N}$-terminal Met-containing C99 fragment of $\beta$ APP (i.e. C100) that potentially could be converted into $\mathrm{C} 99$ by eventual removal of the N-terminal Met. Following C100/C99 generation, it is only a matter of conventional gamma-secretase cleavage to generate $A \beta$ (or Met-A $\beta$ ).

In the bulk of cellular proteins, the translation-initiating Met is removed co-translationally by MAP1 and/or MAP2, and the penultimate encoded amino acid residue becomes the $\mathrm{N}$-terminal residue in the resulting translation product. This is, however, not the case in the production of C100. Unless the penultimate encoded residue is (in order of their size) Gly, Ala, Ser, Cys, Thr, Pro, Val, the translation-initiating Met cannot be removed cotranslationally because the rest of residues would not fit, together with the initiating Met, into active sites of MAP1 and MAP2. In C100, the penultimate residue is Asp, larger than Val; accordingly, its N-terminal Met cannot be removed co-translationally. The $\mathrm{N}$-terminal Met of C100 is, apparently, eventually removed posttranslationally by an aminopeptidase with broad specificity, other than MAP1 or MAP2. It follows that if C100 is continuously produced, its steady-state population should be present in cell despite the removal of N-terminal Met (this is not the case in Alzheimer's brain where in dying cells protein synthesis stops while proteolytic activity continues, i.e. no input of new C100, but active removal of its $\mathrm{N}$-terminal Met and therefore no C100 or Met-A $\beta$ in postmortem samples). The detection of $\mathrm{N}$-terminal Met-containing C99 in a human neuronal cells model of $\mathrm{AD}$ would constitute indisputable proof of the occurrence of $\beta$ APPindependent $A \beta$ generation. Moreover, if the rate of the removal of $\mathrm{N}$-terminal Met from C100 is slower than the rate of its gammasecretase cleavage, a steady state population of $\mathrm{N}$-terminal Metcontaining $A \beta$ should also be present in neuronal cells where the $\beta A P P-i n d e p e n d e n t A \beta$ production pathway has been activated. The occurrence of $\mathrm{N}$-terminal Met in $\mathrm{C} 99$ and in $\mathrm{A} \beta$ would, therefore, function both as an "identifier" of the origin of these molecules and as a reporter of the operation of the $\beta$ APP-independent $A \beta$ generation pathway.

Finally, intracellular retention of the entire output, or the bulk, or a substantial portion of the output of the $\beta$ APP-independent $A \beta$ production pathway, regardless of the enacting mechanism, could be explained by two factors. First is the lack of secretion signal peptide at the $\mathrm{N}$-terminus of $\mathrm{C} 100$ (in contrast to $\beta$ APP where signal peptide is present at the $\mathrm{N}$-terminus). Second, because it is known that in non-neuronal cells $\mathrm{A} \beta$ produced from a transiently expressed C100 can be secreted due to the presence in C100/C99 of the internal trans-membrane domain, it should be assumed that the primary translation product of the $\beta$ APP-independent $A \beta$ generation pathway, C100 (or C99, after removal of N-terminal Met), is processed by gamma-secretase on intracellular, rather than on plasma, membranes, possibly in a neuron-specific manner and is, therefore, retained intraneuronally.

The above notions in general and concepts of the selfpropagating Alzheimer's disease "Engine", illustrated in Figure 16 (as interpreted in the "Alternative Mechanisms" section above), in particular provide guidance for future research and therapeutic strategies for AD. Within the conceptual framework of the present article, the immediate research goals would be testing proposed approaches for activation of the $\beta$ APP-independent $A \beta$ production pathway in human neuronal cells. These approaches could be separated in two conceptual types. One is to switch on the "Starter Motor" with the aim to activate the main $\mathrm{AD}$ "Engine". It involves the importation of $A \beta 42$ to human neuronal cells or its expression from appropriate constructs or transgenes. The other is to bypass the "Starter Motor" and to activate the main AD "Engine" directly. This includes inducing mitochondrial distress and thus activating OMA1 with the goal to trigger operation of the OMA1-DELE1HRI signaling pathway, elicitation of the integrated stress response and consequent activation of one of the mechanisms of $\beta$ APPindependent generation of $A \beta$ described above. Alternatively, the ISR could be elicited more directly, by activation of different eIF2 $\alpha$ kinases with various stressors; this should be tested but on its own may be not sufficient to activate the $\beta$ APP-independent $A \beta$ generation pathway.

The occurrence of $\beta$ APP-independent generation of $A \beta$ in human neuronal cells can be evaluated by testing for the presence of C100 (N-terminal Met-containing C99) or of N-terminal Met-containing $\mathrm{A} \beta$. The detection of either of this species would constitute irrefutable proof of the operation of the $\beta$ APPindependent $A \beta$ production pathway. If such proof is obtained, additional experiments, detailed in the present article, could differentiate between possible underlying mechanisms of $\beta$ APPindependent production of $A \beta$. Importantly, as discussed above, there is good probability that activation of the $\beta$ APP-independent production of intraneuronally retained $A \beta$ in human neuronal cells would trigger formation of neurofibrillary tangles, the major hallmark of Alzheimer's disease. The detection of NFTs would provide a connection to Alzheimer's disease as well as an indication that activation of the $\beta \mathrm{APP}$-independent $\mathrm{A} \beta$ production pathway is required for the development and progression of the disease.

The concepts of the "Starter Motor"-mediated activation of the self-sustaining $\mathrm{AD}$ "Engine" also provide guidance for therapeutic strategies for Alzheimer's disease. It could be reasoned, for example, that the "Starter Motor" can be interfered with by preventing intracellular accumulation of $A \beta$ produced in the $\beta$ APP proteolytic/secretory pathway. This can be potentially achieved with already developed drugs that interfere with proteolytic processing of $\beta$ APP or with levels of secreted $A \beta$ (thus reducing its cellular uptake). Practically, however, this is not feasible. Indeed, to 
be effective, these types of drugs should be administered for very long periods of time, probably many years, prior to the typical timing of manifestation of AD symptoms. Since these types of drugs do not affect operation of the main $\mathrm{AD}$ "Engine" (Figure 16 , as interpreted in the "Alternative Mechanisms" section above), they would be completely inefficient once Alzheimer's disease symptoms manifested, indicating that self-sustaining AD "Engine" has been switched on.

Within the conceptual framework of the present article, the real "treatments" of Alzheimer's disease could only be those drugs that interfere with the operation of the self-perpetuating "Engine" that drives the disease. Such drugs could be administered and be effective even when $\mathrm{AD}$ symptoms already manifested. They could be expected to halt the disease in humans and to reverse and cure it at the early symptomatic stages when the extent of neurodegeneration is not yet substantial. Importantly, the same therapeutic strategies would be indicated regardless of the nature

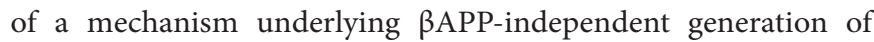
$A \beta$. Potential targets for such drugs include OMA1; its inhibition could effectively stop the AD "Engine". OMA1-mediated cleavage of DELE1 and export of DELE1 fragment from mitochondria to the cytosol could also be targeted, potentially with the same effect. Another set of targets is production of $A \beta$ in the $\beta$ APPindependent pathway and its intraneuronal retention. The latter can be addressed by enacting a small molecule-mediated shift of the location of gamma-secretase cleavage of C100 (or C100-derived C99) from intracellular to plasma membranes and consequent secretion of resulting $A \beta$; this would collapse intraneuronal $\mathrm{A} \beta$ levels and stop the $\mathrm{AD}$ "Engine". As for the suppression of generation of $A \beta$ in the $\beta A P P-i n d e p e n d e n t$ pathway, one highly promising approach, namely the activation (yes, activation rather than inhibition) of beta-secretase, is not only feasible but also illustrative of the drastic differences between the New and the Old Paradigms of Alzheimer's disease.

In closing, the New Paradigm, detailed in the present article, introduces a novel theory of Alzheimer's disease. It provides guidance for the understanding of the disease, suggests conceptually new and defined research and therapeutic strategies, and offers tangible hope for its treatment at the symptomatic stages.

\section{Authors Contributions}

All concepts described in the present article were conceived and developed by the Authors. V.V. wrote the manuscript.

\section{Acknowledgements}

Authors are grateful to Dr. Bjorn R. Olsen for his participation in previous joint publications, which form foundation of the present article, and for helping with preparation of the figures. Authors are also thankful to Michael J. Foley and his associates for logistical support.

\section{Conflicts Of Interest}

Authors declare no conflicts of interest.

\section{Funding}

NIH R21 GM056179; NIH RO1 AR036819.

\section{References}

1. Egan M, Kost J, Tariot P, Paul SA, Cummings JL, Voss L, et al. Randomized trials of verubecestat for mild-to-moderate Alzheimer's disease. N Engl J Med.
2018;378(18):1691-703.

2. Kang J, Lemaire HG, Unterbeck A, Salbaum JM, Masters CL, Grzeschik KH, et al. The precursor of Alzheimer's disease amyloid A4 protein resembles a cell-surface receptor. Nature. 1987;325(6106):733-6.

3. Robakis NK, Ramakrishna N, Wolfe G, Wisniewski HM. Molecular cloning and characterization of a cDNA encoding the cerebrovascular and the neuritic plaque amyloid peptides. Proc Natl Acad Sci U S A. 1987;84(12):4190-4.

4. Tanzi RE, Gusella JF, Watkins PC, Bruns GA, St George-Hyslop P, Van Keuren ML, et al. Amyloid beta protein gene: cDNA, mRNA distribution, and genetic linkage near the Alzheimer locus. Science. 1987;235(4791):880-4.

5. Breimer L, Denny P. Alzheimer amyloid aspects. Nature. 1987;326(6115):749-50.

6. Citron M, Haass C, Selkoe D. Production of amyloid beta peptide by cultured cells: no evidence for internal initiation of translation at Met596. Neurobiol Aging. 1993;14(6):571-3.

7. Macq A, Philippe B, Octave N. The amyloid peptide of Alzheimer's disease is not produced by internal initiation of translation generating C-terminal amyloidogenic fragments of its precursor. Neurosci Lett. 1994;182(2):227-30.

8. Volloch V, Rits-Volloch S. News from Mars: Two-Tier Paradox, Intracellular PCR Chimeric Junction Shift, Dark Matter mRNA and Other Remarkable Features of Mammalian RNA-Dependent mRNA Amplification. Implications for Alzheimer's Disease, RNA-Based Vaccines and mRNA Therapeutics. Ann Integr Mol Med. 2021;2(1):1009. https://www.ncbi.nlm.nih.gov/pmc/articles/PMC8088752/

9. Volloch V. Cytoplasmic Synthesis of Globin RNA in Differentiated Murine Erythroleukemia Cells: Possible Involvement of RNA-dependent RNA Polymerase. Proc Natl Acad Sci USA. 1986;83(5):1208-12.

10. Downey K, Byrnes J, Jurmark B, So A. Reticulocyte RNA-Dependent RNA Polymerase. Proc Natl Acad Sci U S A. 1973;70:3400-4.

11. Volloch V, Schweitzer B, Rits S. Synthesis of Globin RNA in Enucleated Differentiating MEL Cells. J Cell Biol. 1987;105:137-43.

12. Volloch V, Schwetizer B, Rits S. Evolutionarily conserved elements in the 5 untranslated region of beta globin mRNA mediate site-specific priming of a unique hairpin structure during cDNA synthesis. Nucl Acids Res. 1994;22:5302-9.

13. Volloch V, Schweitzer B, Rits S. Antisense Globin RNA in Murine Erythroid Tissues: Structure, Origin and Possible Function. Proc Natl Acad Sci U S A. 1996;93:2476-81.

14. Volloch, V. Determination and characterization of RNA termini by ligationmediated RNA amplification. In: PCR- Essential Techniques. 1997; BIOS, Oxford, J. Burke, editor.

15. Volloch V. Protein-encoding RNA-to-RNA information transfer in mammalian cells: Principles of RNA-dependent mRNA amplification. Ann Integr Mol Med. 2019;1(1):1002. https://www.ncbi.nlm.nih.gov/pmc/articles/PMC6750253/

16. Rits S, Olsen B, Volloch V. Protein-encoding RNA to RNA information transfer in mammalian cells: RNA-dependent mRNA amplification. Identification of chimeric RNA intermediates and putative RNA end products. Ann Integr Mol Med. 2019;1(1):1003. https://www.ncbi.nlm.nih.gov/pmc/articles/PMC6814175/

17. Volloch V, Rits S, Olsen B. RNA-dependent amplification of mammalian mRNA encoding extracellullar matrix proteins: Identification of chimeric RNA intermediates for $\alpha 1, \beta 1$, and $\gamma 1$ chains of laminin. Ann Integr Mol Med. 2019;1(1):1004. https:// www.ncbi.nlm.nih.gov/pmc/articles/PMC6818727/

18. Lai M. RNA replication without RNA-dependent RNA polymerase: surprises from hepatitis delta virus. J Virol. 2005;79:7951-8.

19. Taylor J. Replication of human hepatitis delta virus: recent developments. Trends Microbiol. 2003;11:185-90.

20. Lehmann E, Brueckner F. Cramer P. Molecular basis of RNA-dependent RNA polymerase II activity. Nature. 2007;450:445-9.

21. Wagner S, Yakovchuk P, Ponicsan S, Drullinger L, Kugel J, Goodrich J. RNA polymerase II acts as an RNA-dependent RNA polymerase to extend and destabilize non-coding RNA. EMBO J. 2013;32:781-90. 
22. Maida Y, Yasukawa M, Masutomi K. De Novo RNA Synthesis by RNA-Dependent RNA Polymerase Activity of Telomerase Reverse Transcriptase. Mol Cell Biol 2016;38:1248-59.

23. Kapranov P, Ozsolak F, Kim S, Foissac S, Lipson D, Hart C, et al. New class of genetermini-associated human RNAs suggests a novel RNA copying mechanism. Nature. 2010;466:642-6

24. Tseng C, Lai M. Hepatatis delta virus RNA replication. Viruses. 2009;1:818-31 doi:10.3390/v1030818

25. Brazas R, Ganem D. A cellular homolog of hepatitis delta antigen: implications for viral replication and evolution. Science. 1996;5284:90-4.

26. Huang C, Lo S. Evolution and diversity of human hepatitis D virus genome. Adv Bioinform. 2010;323654.

27. Pakos-Zebrucka K, Koryga I, Mnich K, Mila L, Afshin S, Gorman AM. The integrated stress response. EMBO Rep. 2016;17:1374-95.

28. Ron D. Translational control in the endoplasmic reticulum stress response. J Clin Invest. 2002;110:1383-8.

29. Harding HP, Zhang Y, Zeng H, Novoa I, Lu PD, Calfon M, et al. An integrated stress response regulates amino acid metabolism and resistance to oxidative stress. Mol Cell. 2003;11:619-33.

30. Brostrom CO, Prostko CR, Kaufman RJ, Brostrom MA. Inhibition of translational initiation by activators of the glucose-regulated stress protein and heat shock protein stress response systems. Role of the interferon-inducible double-stranded RNAactivated eukaryotic initiation factor 2alpha kinase. J Biol Chem. 1996;271:24995 25002

31. Dever TE, Feng L, Wek RC, Cigan AM, Donahue TF, Hinnebusch AG Phosphorylation of initiation factor 2 alpha by protein kinase GCN2 mediates genespecific translational control of GCN4 in yeast. Cell. 1992;68:585-96.

32. Wek RC, Jiang HY, Anthony TG. Coping with stress: eIF2 kinases and translational control. Biochem Soc Trans. 2006;34:7-11.

33. Rzymski T, Milani M, Pike L, Buffa F, Mellor HR, Winchester L, et al. Regulation of autophagy by ATF4 in response to severe hypoxia. Oncogene. 2010;29:4424-35.

34. Ye J, Kumanova M, Hart LS, Sloane K, Zhang H, De Panis DN, et al. The GCN2-ATF4 pathway is critical for tumour cell survival and proliferation in response to nutrient deprivation. EMBO J. 2010;29:2082-96.

35. Garcia MA, Meurs EF, Esteban M. The dsRNA protein kinase PKR: virus and cell control. Biochimie. 2007;89:799-811

36. Harding HP, Zhang Y, Ron D. Protein translation and folding are coupled by an endoplasmic-reticulum-resident kinase. Nature. 1999;397:271-4.

37. Berlanga JJ, Herrero S, de Haro C. Characterization of the hemin-sensitive eukaryotic initiation factor 2alpha kinase from mouse nonerythroid cells. J Bio Chem. 1998;273:32340-6.

38. Chen JJ, Throop MS, Gehrke L, Kuo I, Pal JK, Brodsky M, et al. Cloning of the cDNA of the heme-regulated eukaryotic initiation factor 2 alpha (eIF-2 alpha) kinase of rabbit reticulocytes: homology to yeast GCN2 protein kinase and human double-stranded-RNA-dependent eIF-2 alpha kinase. Proc Natl Acad Sci U S A. 1991;88:7729-33.

39. Shi Y, Vattem KM, Sood R, An J, Liang J, Stramm L, et al. Identification and characterization of pancreatic eukaryotic initiation factor 2 alpha-subunit kinase, PEK, involved in translational control. Mol Cell Biol. 1998;18:7499-509.

40. Meurs E, Chong K, Galabru J, Thomas NS, Kerr IM, Williams BR, et al. Molecular cloning and characterization of the human double-stranded RNA-activated protein kinase induced by interferon. Cell. 1990;62:379-90.

41. Ramirez M, Wek RC, Hinnebusch AG. Ribosome association of GCN2 protein kinase, a translational activator of the GCN4 gene of Saccharomyces cerevisiae. Mo Cell Biol. 1991;11:3027-36.

42. Lavoie H, Li JJ, Thevakumaran N, Therrien M, Sicheri F. Dimerization-induced allostery in protein kinase regulation. Trends Biochem Sci. 2014;39:475-86.
43. Donnelly N, Gorman AM, Gupta S, Samali A. The eIF2alpha kinases: their structures and functions. Cell Mol Life Sci. 2013;70:3493-511.

44. Korennykh A, Walter P. Structural basis of the unfolded protein response. Annu Rev Cell Dev Biol. 2012;28:251-77.

45. Wang M, Kaufman RJ. Protein misfolding in the endoplasmic reticulum as a conduit to human disease. Nature. 2016;529:326-35

46. Bertolotti A, Zhang Y, Hendershot LM, Harding HP, Ron D. Dynamic interaction of BiP and ER stress transducers in the unfolded-protein response. Nat Cell Biol. 2000;2:326-32.

47. Carrara M, Prischi F, Nowak PR, Kopp MC, Ali MM. Noncanonical binding of BiP ATPase domain to Ire1 and Perk is dissociated by unfolded protein $\mathrm{CH} 1$ to initiate ER stress signaling. eLife. 2015;4:e03522.

48. Gardner BM, Walter P. Unfolded proteins are Ire1-activating ligands that directly induce the unfolded protein response. Science. 2011;333:1891-4.

49. de la Cadena SG, Hernandez-Fonseca K, Camacho-Arroyo I, Massieu L. Glucose deprivation induces reticulum stress by the PERK pathway and caspase-7- and calpain-mediated caspase-12 activation. Apoptosis. 2014;19:414-27.

50. Moore CE, Omikorede O, Gomez E, Willars GB, Herbert TP. PERK activation at low glucose concentration is mediated by SERCA pump inhibition and confers preemptive cytoprotection to pancreatic beta-cells. Mol Endocrinol. 2011;25:315-26.

51. Hart LS, Cunningham JT, Datta T, Dey S, Tameire F, Lehman SL, et al. ER stressmediated autophagy promotes Myc-dependent transformation and tumor growth. J Clin Invest. 2012;122:4621-34

52. Donnelly N, Gorman AM, Gupta S, Samali A. The eIF2alpha kinases: their structures and functions. Cell Mol Life Sci. 2013;70:3493-511.

53. Vazquez de Aldana CR, Wek RC, Segundo PS, Truesdell AG, Hinnebusch AG. Multicopy tRNA genes functionally suppress mutations in yeast eIF-2 alpha kinase GCN2: evidence for separate pathways coupling GCN4 expression to unchanged tRNA. Mol Cell Biol. 1994;14:7920-32.

54. Castilho BA, Shanmugam R, Silva RC, Ramesh R, Himme BM, Sattlegger E. Keeping the eIF2 alpha kinase Gcn2 in check. Biochim Biophys Acta. 2014;1843:1948-68.

55. Deng J, Harding HP, Raught B, Gingras AC, Berlanga JJ, Scheuner D, et al. Activation of GCN2 in UV-irradiated cells inhibits translation. Curr Biol. 2002;12:1279-86.

56. Lu W, Laszlo CF, Miao Z, Chen H, Wu S. The role of nitric-oxide synthase in the regulation of UVB light-induced phosphorylation of the alpha subunit of eukaryotic initiation factor 2. J Biol Chem. 2009;284:24281-8.

57. Clemens MJ, Elia A. The double-stranded RNA-dependent protein kinase PKR: structure and function. J Interferon Cytokine Res. 1997;17:503-24.

58. Lemaire PA, Anderson E, Lary J, Cole JL. Mechanism of PKR activation by dsRNA. J Mol Biol. 2008;381:351-60.

59. Balachandran S, Roberts PC, Brown LE, Truong H, Pattnaik AK, Archer DR, et al. Essential role for the dsRNA- dependent protein kinase PKR in innate immunity to viral infection. Immunity. 2000;13:129-41.

60. Lee SB, Esteban M. The interferon-induced double-stranded RNA-activated human p68 protein kinase inhibits the replication of vaccinia virus. Virology. 1993;193:103741.

61. Shimazawa M, Hara H. Inhibitor of double stranded RNA-dependent protein kinase protects against cell damage induced by ER stress. Neurosci Lett. 2006;409:192-5.

62. Lee ES, Yoon CH, Kim YS, Bae YS. The double-strand RNA-dependent protein kinase PKR plays a significant role in a sustained ER stress-induced apoptosis. FEBS Lett. 2007;581:4325-32.

63. Garcia MA, Gil J, Ventoso I, Guerra S, Domingo E, Rivas C, et al. Impact of protein kinase PKR in cell biology: from antiviral to antiproliferative action. Microbiol Mol Biol Rev. 2006;70:1032-60.

64. Williams BR. PKR; a sentinel kinase for cellular stress. Oncogene. 1999;18:6112-20.

65. Zhou HR, He K, Landgraf J, Pan X, Pestka JJ. Direct activation of ribosome-associated 
double-stranded RNA-dependent protein kinase (PKR) by deoxynivalenol, anisomycin and ricin: a new model for ribotoxic stress response induction. Toxins. 2014;6:3406-25

66. Reineke LC, Lloyd RE. The stress granule protein G3BP1 recruits protein kinase R to promote multiple innate immune antiviral responses. J Virol. 2015;89:2575-89.

67. Han AP, Yu C, Lu L, Fujiwara Y, Browne C, Chin G, et al. Heme-regulated eIF2alpha kinase (HRI) is required for translational regulation and survival of erythroid precursors in iron deficiency. ЕMBO J. 2001;20:6909-18.

68. Suragani RN, Zachariah RS, Velazquez JG, Liu S, Sun CW, Townes TM, et al. Hemeregulated eIF2alpha kinase activated Atf4 signaling pathway in oxidative stress and erythropoiesis. Blood. 2012;119:5276-84.

69. Ill-Raga G, Tajes M, Busquets-Garcia A, Ramos-Fernandez E, Vargas LM, BoschMorato M, et al. Physiological control of nitric oxide in neuronal BACE1 translation by heme- regulated eIF2alpha kinase HRI induces synaptogenesis. Antioxid Redox Signal. 2015;22:1295-307.

70. McEwen E, Kedersha N, Song B, Scheuner D, Gilks N, Han A, et al. Heme-regulated inhibitor kinase-mediated phosphorylation of eukaryotic translation initiation factor 2 inhibits translation, induces stress granule formation, and mediates survival upon arsenite exposure. J Biol Chem. 2005;280:16925-33.

71. Yerlikaya A, Kimball SR, Stanley BA. Phosphorylation of eIF2alpha in response to $26 \mathrm{~S}$ proteasome inhibition is mediated by the haem-regulated inhibitor (HRI) kinase. Biochem J. 2008;412:579-88.

72. Lu L, Han AP, Chen JJ. Translation initiation control by heme-regulated eukaryotic initiation factor 2alpha kinase in erythroid cells under cytoplasmic stresses. Mol Cel Biol. 2001;21:7971-80

73. Guo X, Aviles G, Tian R. Mitochondrial stress is relayed to the cytosol by an OMA1DELE1-HRI pathway. Nature. 2020;579:427-32.

74. Fessler E, Eckl E, Shmitt S. A pathway coordinated by DELE1 relays mitochondrial stress to the cytosol. Nature. 2020;579:433-7.

75. Volloch V. A mechanism for beta-amyloid overproduction in Alzheimer's disease: precursor-independent generation of beta-amyloid via antisense RNA-primed mRNA synthesis. FEBS Lett. 1996;390:124-8.

76. Volloch V. Mechanism for $\beta$-amyloid overproduction in sporadic Alzheimer's Disease: Possible antisense RNA- mediated generation of a 5'-truncated BAPP mRNA encoding $12 \mathrm{kDa} C$-terminal fragment of $\beta A P P$, the immediate precursor of A $\beta$. In: Molecular Mechanisms of Dementia. 1997, W. Wasco and R. Tanzi, Eds.

77. Volloch V. Possible mechanism for resistance to Alzheimer's disease (AD) in mice suggests new approach to generate a mouse model for sporadic $\mathrm{AD}$ and may explain familial resistance to AD in man. Exp Neurobiol. 1997;144:214-8.

78. Volloch V, Rits S. Results of beta-secretase-inhibitor clinical trials support amyloid precursor protein-independent generation of beta amyloid in sporadic Alzheimer's disease. Med Sci. 2018. doi: 10.3390/medsci6020045.

79. Volloch V, Olsen B, Rits S. Precursor-independent overproduction of beta-amyloid in $\mathrm{AD}$ : Mitochondrial dysfunction as possible initiator of asymmetric RNAdependent $\beta$ APP mRNA amplification. An engine that drives Alzheimer's disease. Ann Integr Mol Med. 2019;1(1):1005. https://www.ncbi.nlm.nih.gov/pmc/articles/ PMC6922309/

80. Volloch V, Olsen B, Rits S. AD "Statin": Alzheimer's disorder is a "fast" disease preventable by therapeutic intervention initiated even late in life and reversible at the early stages. Ann Integr Mol Med. 2020; 2(1):1006. https://www.ncbi.nlm.nih.gov/ pmc/articles/PMC7083596/

81. Volloch V, Olsen B, Rits S. Alzheimer's Disease is Driven by Intraneuronally Retained Beta-Amyloid Produced in the AD-Specific, $\beta$ APP-Independent Pathway: Current Perspective and Experimental Models for Tomorrow. Ann Integr Mol Med. 2020;2(1):1007. https://www.ncbi.nlm.nih.gov/pmc/articles/PMC7331974/

82. Volloch V, Olsen B, Rits S. Alzheimer's disease prevention and treatment: Case for optimism. Ann Integr Mol Med. 2020;2(1):1008. https://www.ncbi.nlm.nih.gov/ pmc/articles/PMC7546530/
83. Yoshida H, Matsui T, Yamamoto A, Okada T, Mori K. XBP1 mRNA is induced by ATF6 and spliced by IRE1 in response to ER stress to produce a highly active transcription factor. Cell. 2001;107(7):881-91.

84. Jiang S, Zhang E, Zhang R, Li X. Altered activity patterns of transcription factors induced by endoplasmic reticulum stress. BMC Biochem. 2016;17:8.

85. Liang Y, Song Y, Zhang F, Sun F, Wag N. Effect of a Single Nucleotide Polymorphism in the LAMA1 Promoter Region on Transcriptional Activity: Implication for Pathological Myopia. Curr Eye Res. 2016;10:1379-86.

86. Piccinni S, Bolcato-Bellemin A, Klein A. Kruppel-like factors regulate the Lamal gene encoding the laminin chain. J Biol Chem. 2004;279:9103-14.

87. Okano R, Mita T, Matsui T. Characterization of a novel promoter structure and its transcriptional regulation of the murine laminin B1 gene. Biochem Biophys Acta. 1992;1132:49-57.

88. Lodish H, Berk A, Zipurski A. In: Molecular Cell Biology. New York, 2000, Freeman and Co.

89. Kearse M, Wilusz J. Non-AUG translation: a new start for protein synthesis in eukaryotes. Genes Dev 2017;31:1717-31.

90. Cao A, Galanello R. Beta-thalassemia. Genet Med. 2010;12:61-76.

91. Mita S, Sadlock J, Herbert J, Schon E. A cDNA specifying the human amyloid beta precursor protein encodes a 95-kDa polypeptide. Nucl Acids Res. 1988;16:9351.

92. Mita S, Sadlock J, Herbert J, Schon E. A cDNA specifying the human amyloid beta precursor protein encodes a $95-\mathrm{kDa}$ polypeptide: Correction. Nucl Acids Res. $1988 ; 16: 11402$.

93. Salbaum J, Weidemann A, Lemaire H, Masters C, Beyreuther K. The promoter of Alzheimer's disease amyloid A4 precursor gene. ЕMBO J. 1988;7:2807-13.

94. Zhu X, Perry G, Moreira PI, Aliev G, Cash AD, Hirai K, et al. Mitochondrial abnormalities and oxidative imbalance in Alzheimer disease. J Alzheimers Dis. 2006;9:147-53.

95. Blass JP. The mitochondrial spiral. An adequate cause of dementia in the Alzheimer's syndrome. Ann N Y Acad Sci. 2000;924:170-83.

96. Manczak M, Park BS, Jung Y, Reddy PH. Differential expression of oxidative phosphorylation genes in patients with Alzheimer's disease: implications for early mitochondrial dysfunction and oxidative damage. Neuromolecular Med. 2004;5:147-62.

97. Qin W, Haroutunian V, Katsel P, Cardozo CP, Ho L, Buxbaum JD, et al. PGC-1alpha expression decreases in the Alzheimer disease brain as a function of dementia. Arch Neurol. 2009;66:352-61.

98. Du H, Guo L, Yan S, Sosunov AA, McKhann GM, Yan SS. Early deficits in synaptic mitochondria in an Alzheimer's disease mouse model. Proc Natl Acad Sci U S A. 2010;107:18670-5.

99. Lin MT, Simon DK, Ahn CH, Kim LM, Beal MF. High aggregate burden of somatic mtDNA point mutations in aging and Alzheimer's disease brain. Hum Mol Genet. 2002;11:133-45.

100. Calkins M, Manczak M, Mao P, Shirendeb U, Reddy PH. Impaired mitochondrial biogenesis, defective axonal transport of mitochondria, abnormal mitochondrial dynamics and synaptic degeneration in a mouse model of Alzheimer's disease. Hum Mol Genet. 2011;20(23):4515-29.

101. Anandatheerthavarada HK, Biswas G, Robin MA, Avadhani NG. Mitochondrial targeting and a novel transmembrane arrest of Alzheimer's amyloid precursor protein impairs mitochondrial function in neuronal cells. J Cell Biol. 2003;161:4154.

102. Caspersen C, Wang N, Yao J. Mitochondrial Abeta: a potential focal point for neuronal metabolic dysfunction in Alzheimer's disease. FASEB J. 2005;19:2040-1.

103. Chen JX, Yan SS. Role of mitochondrial amyloid-beta in Alzheimer's disease. J Alzheimers Dis. 2010;20:S569-78.

104. Hansson C, Petersen CA, Alikhani N, Behbahani H. The amyloid beta-peptide is imported into mitochondria via the TOM import machinery and localized to mitochondrial cristae. Proc Natl Acad Sci U S A. 2008;105:13145-50. 
105. de la Monte SM, Luong T, Neely TR, Robinson D, Wands JR. Mitochondrial DNA damage as a mechanism of cell loss in Alzheimer's disease. Lab Invest. 2000;80:1323 5 .

106. Brooks WM, Lynch PJ, Ingle CC, Hatton A, Emson PC, Faull RL, Starkey MP. Gene expression profiles of metabolic enzyme transcripts in Alzheimer's disease. Brain Res. 2007;1127:127-35

107. Wang X, Perry G, Smith MA, Zhu X. Amyloid-beta-derived diffusible ligands cause impaired axonal transport of mitochondria in neurons. Neurodegener Dis. 2007;7:56-9.

108. Wang X, Su B, Fujioka H, Zhu X. Dynamin-like protein 1 reduction underlies mitochondrial morphology and distribution abnormalities in fibroblasts from sporadic Alzheimer's disease patients. Am J Pathol. 2008;173:470-82.

109. Wang X, Su B, Lee HG, Li X, Perry G, Smith MA, et al. Impaired balance of mitochondrial fission and fusion in Alzheimer's disease. J Neurosci. 2009;29:9090103.

110. Wang X, Su B, Siedlak SL, Moreira PI, Fujioka H, Wang Y, et al. Amyloidbeta overproduction causes abnormal mitochondrial dynamics via differential modulation of mitochondrial fission/fusion proteins. Proc Natl Acad Sci U S A 2008;105:19318-23.

111. Chafekar S, Baas F, Scheper W. Oligomer-specific amyloid-beta toxicity in cell models is mediated by selective uptake. Biochem Biophys Acta. 2008;9:523-31.

112. Wesen E, Jeffries G, Dzebo M, Esbjorner M. Endocytic uptake of monomeric amyloid- $\beta$ peptides is clathrin- and dynamin-independent and results in selective accumulation of $A \beta(1-42)$ compared to $A \beta(1-40)$. Sci Rep. 2017;7:2021.

113. Hu X, Crick S. Amyloid seeds formed by cellular uptake, concentration, and aggregation of the amyloid-beta peptide. Proc Natl Acad Sci USA. 2009;106:20324-9.

114. Yajima R, Tokutake T, Koyama A. ApoE-isoform-dependent cellular uptake of amyloid- $\beta$ is mediated by lipoprotein receptor LR11/SorLA. Biochem Biophys Res Comm. 2015;456:482-8.

115. Omtri R, Davidson M, Arumugam B. Differences in the cellular uptake and intracellular itineraries of amyloid beta proteins 40 and 42: ramifications for the Alzheimer's drug discovery. Mol Pharmaceutics. 2012;9:1887.

116. Bu G, Cam J, Zerbinatti C. LRP in amyloid-beta production and metabolism. Ann NY Acad Sci. 2006;086:35-53.

117. Wang $\mathrm{H}$, et al. beta-Amyloid(1-42) binds to alpha7 nicotinic acetylcholine recepto with high affinity. Implications for Alzheimer's disease pathology. J Biol Chem. 2000;275:5626-32.

118. Nagele R, D'Andrea, M, Anderson, W, Wang H. Intracellular accumulation of betaamyloid(1-42) in neurons is facilitated by the alpha 7 nicotinic acetylcholine receptor in Alzheimer's disease. Neuroscience. 2002;110:199-211.

119. Oddo $\mathrm{S}$. Chronic nicotine administration exacerbates tau pathology in a transgenic model of Alzheimer's disease. Proc Natl Acad Sci USA. 2005;102:3046-51.

120. Yan S. RAGE and amyloid-beta peptide neurotoxicity in Alzheimer's disease. Nature. 1996;382:685-91

121. Sasaki N. Immunohistochemical distribution of the receptor for advanced glycation end products in neurons and astrocytes in Alzheimer's disease. Brain Res. 2001;888:256-62.

122. Deane R. RAGE mediates amyloid-beta peptide transport across the blood-brain barrier and accumulation in brain. Nature Med. 2003;9:907-13.

123. Iribarren P, Zhou Y, Hu J, Le Y, Wang, J. Role of formyl peptide receptor-like 1 (FPRL1/FPR2) in mononuclear phagocyte responses in Alzheimer disease. Immunol Res. 2005;31:165-76.

124. Snyder E. Regulation of NMDA receptor trafficking by amyloid- $\beta$. Nature Neurosci. 2005;8:1051-8.

125. LaFerla F, Green K, Oddo S. Intracellular amyloid-beta in Alzheimer's disease. Nat Rev Neurosci. 2007;8(7):499-509.

126. Kinoshita A. Demonstration by FRET of BACE interaction with the amyloid precursor protein at the cell surface and in early endosomes. J Cell Sci. 2003;116:3339-46.

127. Xu H, Greengard P, Gandy S. Regulated formation of Golgi secretory vesicles containing Alzheimer beta-amyloid precursor protein. J Biol Chem. 1995;270:232435

128. Mizuguchi M, Ikeda K, Kim S. Differential distribution of cellular forms of betaamyloid precursor protein in murine glial cell cultures. Brain Res. 1992;584:219-25.

129. Cook D. Alzheimer's $A \beta(1-42)$ is generated in the endoplasmic reticulum/ intermediate compartment of NT2N cells. Nature Med. 1997;3:1021-3.

130. Hartmann T. Distinct sites of intracellular production for Alzheimer's disease A $340 / 42$ amyloid peptides. Nature Med. 1997;3:1016-20.

131. Wild-Bode C. Intracellular generation and accumulation of amyloid beta-peptide terminating at amino acid 42. J Biol Chem. 1997;272:16085-8.

132. Lee $S$. A detergent-insoluble membrane compartment contains $A \beta$ in vivo. Nature Med. 1998;4:730-4.

133. Skovronsky D, Doms R, Lee V. Detection of a Novel Intraneuronal Pool of Insoluble Amyloid $\beta$ Protein that Accumulates with Time in Culture. J Cell Biol. 1998;141:1031-9.

134. Manczak M. Mitochondria are a direct site of A beta accumulation in Alzheimer's disease neurons: implications for free radical generation and oxidative damage in disease progression. Hum Mol Genet. 2006;15:1437-49.

135. Sannerud R. Restricted Location of PSEN2/gamma-secretase determines substrate specificity and generates an intracellular Abeta pool. Cell. 2016;166:193-208.

136. Hansson C. Nicastrin, presenilin, APH-1, and PEN-2 form active gamma-secretase complexes in mitochondria. J Biol Chem. 2004;279:51654-60.

137. Caspersen C. Mitochondrial Abeta: a potential focal point for neuronal metabolic dysfunction in Alzheimer's disease. FASEB J. 2005;19:2040-1

138. Dyrks T, Dyrks E, Monning U, Urmoneit B, Turner J, Beyreuther, K. Generation of beta A4 from the amyloid protein precursor and fragments thereof. FEBS Let. 1993;335:89-93

139. Iizuka T, Shoji M, Kawarabayashi T, Sato M, Kobayashi T. Intracellular generation of amyloid beta-protein from amyloid beta-protein precursor fragment by direct cleavage with beta- and gamma-secretase. Biochem. Biophys. Res Commun. 1996;218:238-42.

140. Mullan M, Crawford F, Axelman K, Houlden H, Lilius L, Winblad B, et al. A pathogenic mutation for probable Alzheimer's disease in the APP gene at the N-terminus of beta-amyloid. Nat Genet.1992;1(5):345-7.

141. Haass C, Lemere C, Capell A, Citron M, Seubert P, Schenk D, et al. The Swedish mutation causes early- onset Alzheimer's disease by beta-secretase cleavage within the secretory pathway. Nat Med. 1995;1(12):1291-6.

142. O'Brien R, Wong P. Amyloid precursor protein processing and Alzheimer's disease Annu Rev Neurosci. 2011;34:185-204.

143. Martin B, Schrader-Fischer G, Busciglio J, Duke M, Paganetti P, Yankner B. Intracellular accumulation of beta- amyloid in cells expressing the Swedish mutant amyloid precursor protein. J Biol Chem. 1995;270:26727-30.

144. Teo E, Ravi S, Barardo D, Kim H, Fong S. Metabolic stress is a primary pathogenic event in transgenic Caenorhabditis elegans expressing pan-neuronal human amyloid beta. eLife. 2019.

145. Keskin A, Kekuš M, Adelsberger H, Neumann U, Shimshek D. BACE inhibitiondependent repair of Alzheimer's pathophysiology. Proc Natl Acad Sci USA. 2017;114:8631-6.

146. Hu X, Das B, Hou H, He W, Yan R. BACE1 deletion in the adult mouse reverses preformed amyloid deposition and improves cognitive functions. J Exp Med. 2018;10:1084

147. Varland S, Osberg C, Arnesen T. N-terminal modifications of cellular proteins: The enzymes involved, their substrate specificities and biological effects. Proteomics. 2015;15(14):2385-401 
148. Frottin F, Martinez A, Peinot P, Mitra S, Holz R, Giglione C, Meinnel T. The proteomics of N-terminal methionine cleavage. Mol Cell Proteomics. 2006;5(12):2336-49.

149. Xiao Q, Zhang F, Nacev B, Liu J, Pei D. Protein N-Terminal Processing: Substrate Specificity of Escherichia coli and Human Methionine Aminopeptidases. Biochemistry. 2010;49:5588-99.

150. Varshavsky A. The N-end rule pathway and regulation by proteolysis. Protein Sci. 2011;20:1298-45

151. Wingfield P. N-Terminal Methionine Processing. Curr Protoc Protein Sci. 2017;88:1 6.

152. Polevoda B, Sherman F. N-terminal acetyltransferases and sequence requirements for N-terminal acetylation of eukaryotic proteins. J Mol Biol. 2003;325:595-622.

153. Choi S. A three-dimensional human neural cell culture model of Alzheimer's disease Nature. 2014;515:274-8.

154. Girardin S, Griparic L, Cuziol C, Philpott D, Arnoult D. The eIF2 $\alpha$ kinase HRI in innate immunity, proteostasis, and mitochondrial stress. FEBS J. 2021;288:3094-107.

155. Head B, Griparic L, Amiri M, Gandre-Babbe S, van der Bliek A. Inducible proteolytic inactivation of OPA1 mediated by the OMA1 protease in mammalian cells. J. Cell Biol. 2009;187:959-66.

156. Ehses S, Raschke I, Mancuso G, Bernacchia A, Geimer A, Tondera D, et al. Regulation of OPA1 processing and mitochondrial fusion by m-AAA protease isoenzymes and OMA1. J Cell Biol, 2009;187:1023-36.

157. McBride H, Soubannier B. Mitochondrial Function: OMAl and OPAl, the Grandmasters of Mitochondrial Health. Curr Biol. 2010;20:274-6.

158. Baker M, Lampe P, Stojanovski D. Stress-induced OMA1 activation and autocatalytic turnover regulate OPA1- dependent mitochondrial dynamics. EMBO J. 2014;33:57893.

159. Gilkerson R, DeLa Torre P, St.Vallier S. Front. Mitochondrial OMA1 and OPA1 as Gatekeepers of Organellar Structure/Function and Cellular Stress Response. Cell Dev Biol. 2021. https://doi.org/10.3389/fcell.2021.626117

160. Tanzi R, Bertram L. Twenty years of the Alzheimer's disease amyloid hypothesis: a genetic perspective. Cell. 2005;120:545-55.

161. Hardy J, Selkoe D. The amyloid hypothesis of Alzheimer's disease: progress and problems on the road to therapeutics. Science. 2002;297:353-6.

162. Selkoe D. Alzheimer's disease is a synaptic failure. Science. 2002;298:789-91.

163. Duff K. Transgenic mouse models of Alzheimer's disease: phenotype and mechanisms of pathogenesis. Biochem Soc Symp. 2001;67:195-202.

164. Chin J. Selecting a mouse model of Alzheimer's disease. Methods Mol Biol. 2011;670:169-89.

165. Choi S, Tanzi R. iPSCs to the rescue in Alzheimer's research. Cell Stem Cell. 2012;10:235-6.

166. Yagi T. Modeling familial Alzheimer's disease with induced pluripotent stem cells. Human Mol Genet. 2011;20:4530-9.

167. Israel M. Probing sporadic and familial Alzheimer's disease using induced pluripotent stem cells. Nature. 2012;482:216-20.

168. Kondo T. Modeling Alzheimer's disease with iPSCs reveals stress phenotypes associated with intracellular $A \beta$ and differential drug responsiveness. Cell Stem Cell. 2013;12:487-96.

169. Muratore C. The familial Alzheimer's disease APPV717I mutation alters APP processing and Tau expression in iPSC-derived neurons. Hum Mol Genet. 2014;23:3523-6.

170. Sproul A. Characterization and Molecular Profiling of PSEN1 Familial Alzheimer's Disease iPSC-Derived Neural Progenitors. PLoS ONE. 2014;9:e84547.

171. Almeida C, Takahashi, R, Gouras G. $\beta$-amyloid accumulation impairs multivesicula body sorting by inhibiting the ubiquitin-proteasome system. J. Neurosci. 2006;26:4277-88.
172. Gregori L, Fuchs C, Figueiredo-Pereira M, Van Nostrand W, Goldgaber D. Amyloid $\beta$-protein inhibits ubiquitin- dependent protein degradation in vitro. J Biol Chem. 1995;270:19702-8.

173. Oh S. Amyloid peptide attenuates the proteasome activity in neuronal cells. Mech Ageing Dev. 2005;126:1292-9.

174. Tseng B, Green K, Chan J, Blurton-Jones M, LaFerla F. A $\beta$ inhibits the proteasome and enhances amyloid and tau accumulation. Neurobiol Aging. 2008;29:1607-18.

175. Mukherjee S, Perez K, Lago L. Quantification of N-terminal amyloid- $\beta$ isoforms reveals isomers are the most abundant form of the amyloid- $\beta$ peptide in sporadic Alzheimer's disease. Brain Commun. 2021;3(1). https://doi.org/10.1093/ braincomms/fcab028

176. Kukar T, Ladd T, Bann A. Substrate-targeting $\gamma$-secretase modulators. Nature. 2008;453:925-9.

177. Bursavich M, Harrison B, Blain J. Gamma Secretase Modulators: New Alzheimer's Drugs on the Horizon? J Med Chem. 2016;59:7389-409.

178. Crump C, Johnson D, Li Y. Development and Mechanism of $\gamma$-Secretase Modulators for Alzheimer Disease. Biochemistry. 2013;52:10.

179. Wolfe M. Gamma-secretase modulators. Curr Alzheimer Res. 2007;4:571-3.

180. Golde T, Koo E, Felsenstein K, Osborne B, Miele L. $\gamma$-Secretase inhibitors and modulators. BBA Biomembranes. 2013;12:2898-907.

181. Lessard CB, Rodriguez E, Ladd TB. $\gamma$-Secretase modulators exhibit selectivity for modulation of APP cleavage but inverse $\gamma$-secretase modulators do not. Alz Res Ther. 2020;12:61.

182. Dobrowolska J, Vassar R. A promising new $\gamma$-secretase modulator for Alzheimer's disease. J Exp Med. 2021;218(4):e20210077.

183. Mekala S, Nelson G, Li Y. Recent developments of small molecule $\gamma$-secretase modulators for Alzheimer's disease. RSC Medicinal Chem. 2020;9.

184. Huse J, Liu K, Pijak D, Carlin D, Lee V Doms R. Beta-secretase processing in the trans-Golgi network preferentially generates truncated amyloid species that accumulate in Alzheimer's disease brain. J Biol Chem. 2002;277:16278-84.

185. Liu K, Doms R, Lee M. Glu11 site cleavage and N-terminally truncated A beta production upon BACE overexpression. Biochemistry. 2002;41:3128-36.

186. Lee E, Zhang B, Liu K. BACE overexpression alters the subcellular processing of APP and inhibits $A \beta$ deposition in vivo. J Cell Biol. 2005;168:291-302.

187. Kimura A, Hata S, Suzuki T. Alternative selection of beta-site APP-cleaving enzyme 1 (BACE1) cleavage sites in amyloid beta-protein precursor (APP) harboring protective and pathogenic mutations within the Abeta sequence. J Biol Chem. 2016;291:24041-53

188. Fluhrer R, Multhaup G, Schlicksupp A, Okochi M. Takeda M, Lammich S, et al. Identification of a beta-secretase activity, which truncates amyloid beta-peptide after its presenilin-dependent generation. J Biol Chem. 2003;278:5531-8.

189. Shi X, Tugusheva K, Bruce J, Lucka A, Wu G, Chen-Dodson E, et al. Beta-secretase cleavage at amino acid residue 34 in the amyloid beta peptide is dependent upon gamma-secretase activity. J Biol Chem. 2003;278:21286-94.

190. Liebsch F, Kulic L, Teunissen C, Shobo A, Ulku I, Engelschalt V, et al. A 334 is a BACE1-derived degradation intermediate associated with amyloid clearance and Alzheimer's disease progression. Nat Commun. 2019;10:2240.

191. Garcia-Gonzales L, Pilat D, Rivera S. Emerging Alternative Proteinases in APP Metabolism and Alzheimer's Disease Pathogenesis. Front Aging Neurosci. 2019. https://doi.org/10.3389/fnagi.2019.00244

192. Conan-Doyle A. The sign of the four. Lippincott's Monthly Magazine. 1890. 